\title{
POPULATION AND IMMUNOCOMPETENT GENETIC VARIATION: A FIELD-BASED STUDY
}

\author{
A thesis presented for the degree \\ Doctor of Philosophy
}

Amber Teacher

School of Biological and Chemical Sciences, Queen Mary, University of London

\&

Institute of Zoology, Zoological Society of London

Submitted December 2008 


\section{Statement of Originality}

I certify that this thesis, and the research to which it refers, are the product of my own work (apart from the simulations as noted in chapters 5 and 6), and that any idea or quotations from the work of other people, published or otherwise, are fully acknowledged in accordance with the standard referencing practices of the discipline. I acknowledge the helpful guidance and support of my supervisors, Dr Trenton Garner, Prof Richard Nichols and Dr Andrew Cunningham.

Signed: 


\section{Acknowledgements}

I would particularly like to thank Trent Garner, Richard Nichols and Andrew Cunningham for their invaluable advice and support throughout. I'm also very grateful to Seirian Sumner and Kate Ciborowski for teaching me all things lab-related before I started this work, and to Dada Gotelli for keeping the genetics lab running so smoothly. This project could not have taken place without all the pond owners who allowed access to their frog populations, nor without Jules Howard and Froglife (registered charity no.1093372) who shared records of amphibian mortalities, and the Irish Peatlands Conservation Council (registered charity no. CHY6829) who assisted with locating study populations in Ireland.

Thanks to my panel members at Queen Mary, Rob Knell and Jon Grey, for reading preliminary reports and giving feedback that undoubtedly improved this work. Particular thanks to Bill Jordan for advice in the lab that made this work as rigorous as it could be. Thanks also to Andrew King for assistance with Generalised Linear Modelling, Jinliang Wang for helpful discussions on relatedness and inbreeding, to Chikako Matsuba and Juha Merilä for advice on phylogeography, and to Inga Zeisset and Shoshanna May for their experience on amphibian MHC genes. Special thanks to Dave Griffiths for constant support over the years, as well as for writing marvellous data manipulation programs, and also to Paddy O'Byrne and Michèle Bland for their ongoing support and also proof reading much of this thesis. Thanks also, of course, to the perfect office mates, Andy King and Jon Bielby - both have given ideas and opinions on parts of this thesis.

Many volunteers contributed their time and enthusiasm to this project both in the lab and in the field. Particular thanks to Sayako Arai for her brilliant laboratory assistance, running plates of samples for microsatellite analysis. Thanks also to Miriam Isabel Smith for fieldwork and lab assistance, to Amanda Duffus for fieldwork assistance and for sharing disease screening results, and to Michael Williamson, Elizabeth Webber, 
Jennifer Gilbert, Deise Nishimura, Nadia Sitas, Rhiannon Lloyd and Mimi Sun for fieldwork assistance. Many thanks also to those who provided samples from their archives: Chikako Matsuba, Juha Merila, Robert Jehle, Jaime Bosch, Dirk Schmeller, Miguel Vences, Wieslaw Babik, Pim Artzen, Olga Tsinenko, Josh van Buskirk, Christophe Eggert and Benedikt Schmidt.

All fieldwork in the Republic of Ireland was performed under licensing from the National Parks and Wildlife Service, and animal work in the UK was performed under UK Home Office licensing, and ethics committee approval from the Zoological Society of London. This work was funded by a Natural Environment Research Council studentship with CASE support from Herpetofauna Consultants International Ltd. and additional funding from the Zoological Society of London, the University of London Central Research Fund, the Universities Federation for Animal Welfare, the British Wildlife Health Association, and the Genetics Society. 


\section{Abstract of Thesis}

In order to establish an evolutionary context, this thesis examines the phylogeography of the common frog (Rana temporaria) throughout Europe, with a particular focus on the British Isles. Mitochondrial DNA indicates divergent, yet overlapping Eastern and Western European haplotypes, and that Britain was colonized via Western Europe, likely from an Iberian glacial refugium. Ireland appears to have been colonized both via this route, and from a refugium in the South West of Ireland. Data from nuclear microsatellite markers indicate that although the mtDNA haplotypes in England and Eastern Ireland are indistinguishable, there is vastly lower genetic diversity in Ireland.

Moving to a finer scale, common frogs in Britain are examined for differences in population genetic patterns that may have been caused by disease outbreaks (Ranavirus). Populations that have experienced recurrent mortalities for approximately five generations are compared to those with no history of disease. Populations that suffer recurrent disease are shown to have experienced approximately $80 \%$ declines in the numbers of frogs ten years after the disease first arrived. Yet surprisingly, microsatellite markers show no signal of reduced genetic variability; it is possible that immigration has maintained allelic richness in infected populations. An excess of homozygosity, and a reduction of relatedness within infected ponds implies that assortative mating may be occurring, and population genetic simulations confirm that assortative mating can produce these patterns.

A novel Major Histocompatibility Complex (a region directly involved in the immune response to viruses) marker was developed, and haplotype frequencies are shown to differ between infected and uninfected populations. Infected populations show reduced genetic diversity and more uniform allelic frequencies; implying directional selection for more resistant genotypes. These findings indicate that Ranavirus may be affecting the population dynamics of the common frog, and that this species appears to be adapting to the presence of the disease. 


\section{Table of Contents}

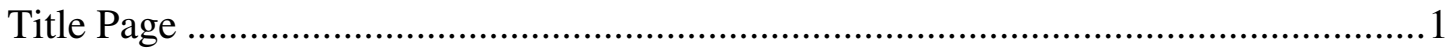

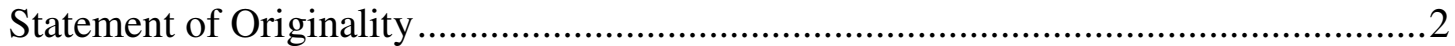

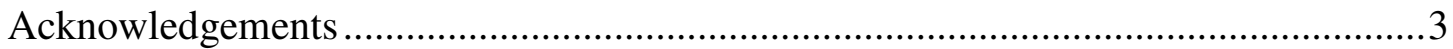

Abstract of Thesis ......................................................................................... 5

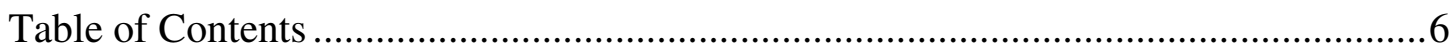

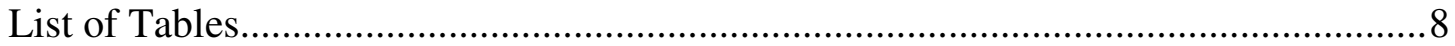

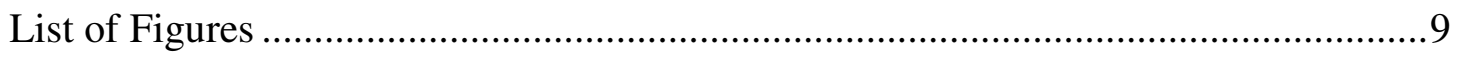

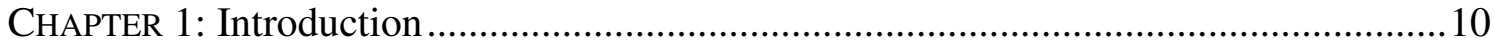

CHAPTER 2: European phylogeography of the common frog (Rana temporaria), and postglacial colonisation routes into the British Isles................................................ 21

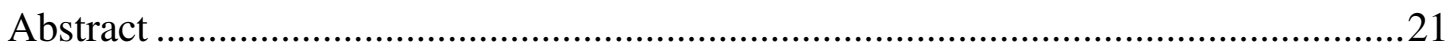

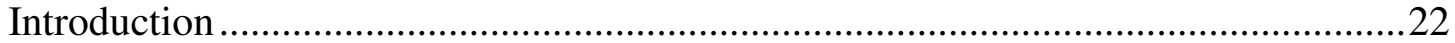

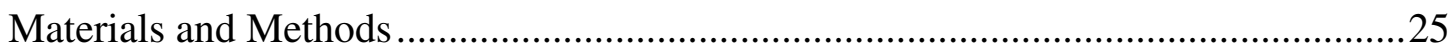

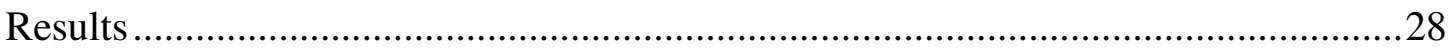

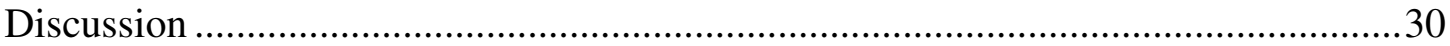

CHAPTER 3: Microsatellite analysis reveals dramatic differences in population genetic variation of the common frog (Rana temporaria) in England and Ireland ......................34

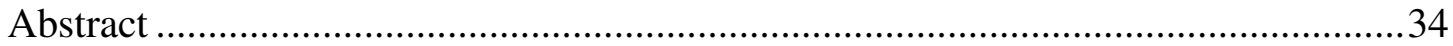

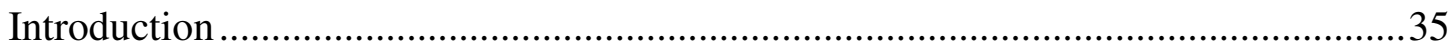

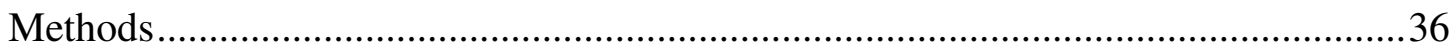

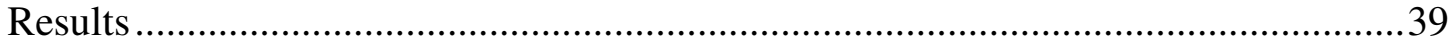

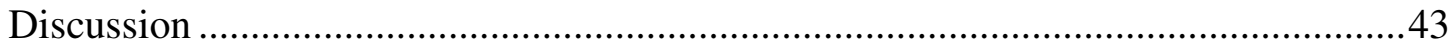

CHAPTER 4: The impact of Ranavirus infection on common frog populations in the UK

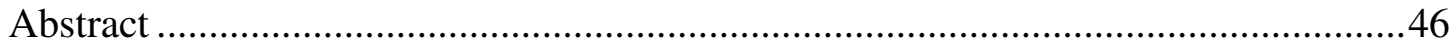

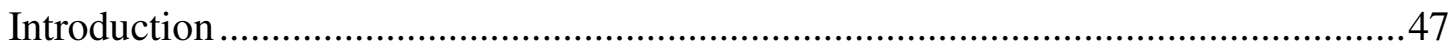

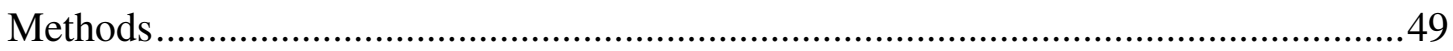

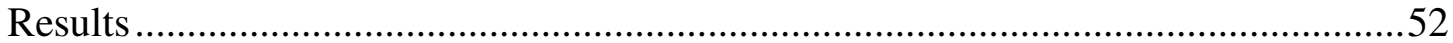


Discussion .56

CHAPTER 5: Neutral genetic variation in wild populations of common frogs (Rana temporaria) which have been subject to disease outbreaks (Ranavirus).......................60

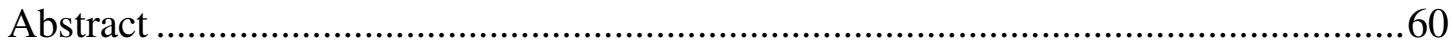

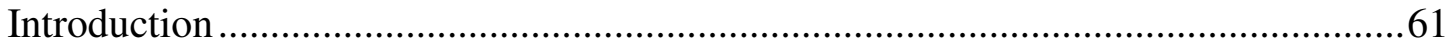

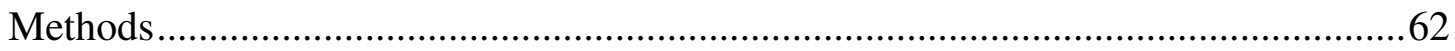

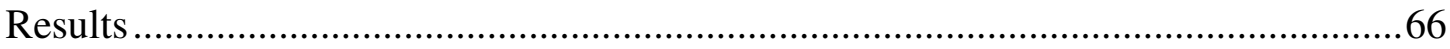

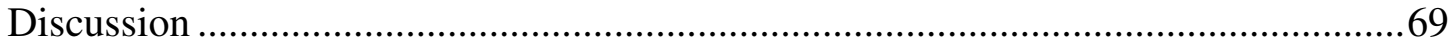

CHAPTER 6: Adaptive genetic variation in wild populations of common frogs (Rana temporaria) which have been subject to disease outbreaks (Ranavirus).......................73

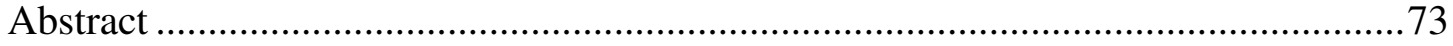

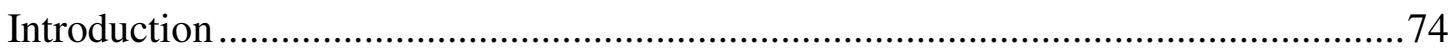

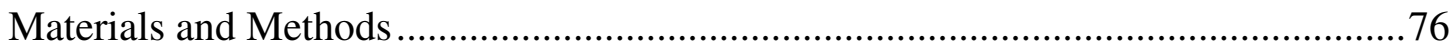

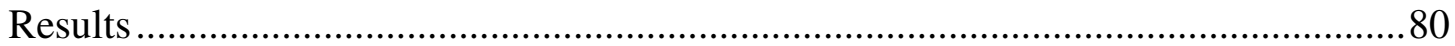

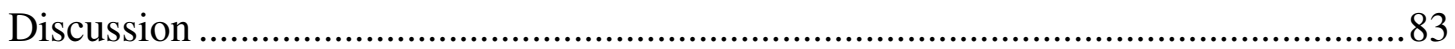

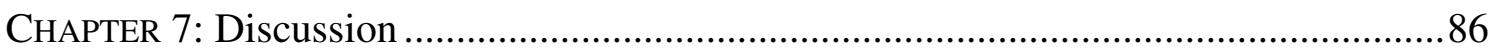

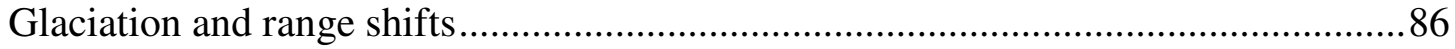

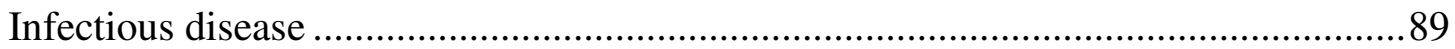

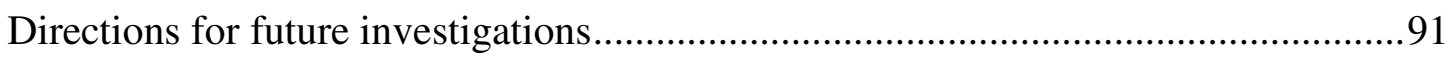

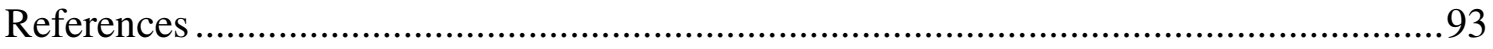

Appendix 1 -Protocol for cleaning sequencing reaction products .............................107

Appendix 2 - Polymorphic sites at each mtDNA haplotype..................................... 108

Appendix 3 - Simulation code for use in R package ............................................... 109

Appendix 4 - R code for randomization of disease status and generation of Chi-squared

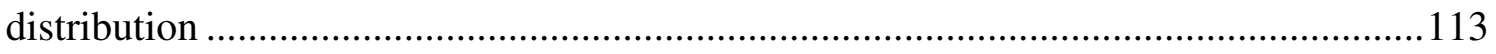

Appendix 5 - Parliamentary Office of Science and Technology note ..........................114 


\section{List of Tables}

CHAPTER 3:

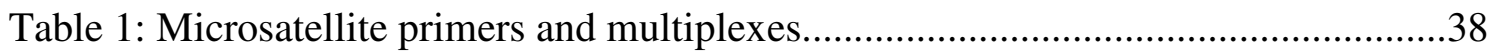

CHAPTER 4:

Table 1: Details of adult frogs examined for signs of Ranavirus infection......................53

Table 2: Results of diagnostic tests for the presence of Ranavirus in frog carcasses......54

CHAPTER 5:

Table 1: Comparison of genetic variation in infected and uninfected populations.........67

Table 2: Results from population simulations....................................................68 


\section{List of Figures}

\section{CHAPTER 2}

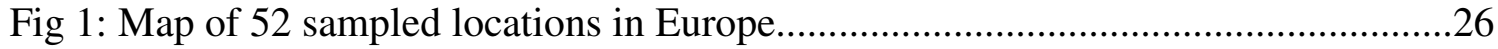

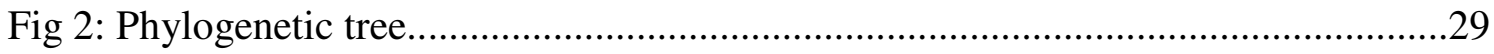

Fig 3: Haplotype distribution in Europe ................................................................ 30

CHAPTER 3

Fig 1: Map showing the location of populations sampled in England and Ireland..........37

Fig 2: Allelic richness at each locus averaged across population groups.......................41

Fig 3: Observed heterozygosity at each locus averaged across population groups..........41

Fig 4: Box and whisker plot showing the distribution of allelic richness within

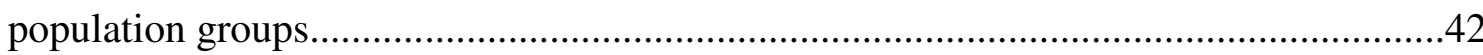

Fig 5: Box and whisker plot showing the distribution of observed heterozygosity within

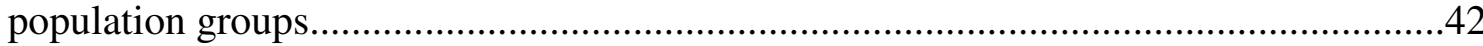

Fig 6: Correspondence analysis of English and Irish populations.............................43

CHAPTER 4

Fig 1: Lesions characteristic of ulcerative disease syndrome caused by infection with

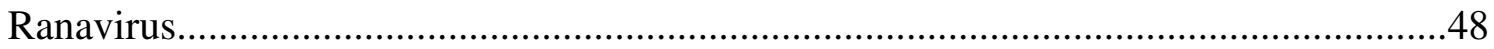

Fig 2: Scarring characteristic of healed skin ulceration.........................................48

Fig 3: Numbers of frogs killed in Ranavirus mass mortality events.............................55

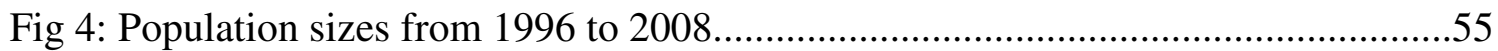

\section{CHAPTER 5}

Fig 1: Map of sampling sites in England

Fig 2: Simulated $\mathrm{F}_{\mathrm{IS}}$ under random and assortative mating.

CHAPTER 6

Fig 1: Map showing the location of study sites in England.....................................77

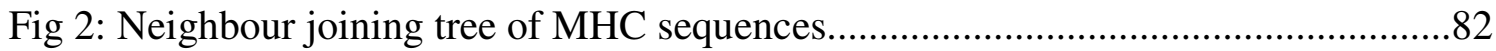

Fig 3: Distribution of Chi-squared values generated by randomising disease status.......83 


\section{CHAPTER 1: Introduction}

This thesis examines how wild populations of common frogs have been affected by both historical and recent crises. In particular, I use genetic data from nuclear microsatellite loci, a Major Histocompatibility Complex (MHC) locus, and mitochondrial DNA to infer the influence of historical glaciation events and a recent disease outbreak on the genetic patterns present in extant populations. The evolutionary processes underlying the observed genetic patterns are discussed, and theoretical expectations are compared with empirical data. Each chapter contains its own introduction and discussion specific to the questions posed and so this general introduction serves to present some of the theory which underlies the thesis as a whole.

\section{Why common frogs?}

Common frogs (Rana temporaria) are an ideal study species for the impacts of European glaciations as they are found throughout Europe and are widespread in the UK and Ireland. Sampling is relatively straightforward, and populations easily identifiable, as common frogs can be found in almost any habitat where there is a small to medium sized permanent body of fresh water. Garden ponds represent important habitat in the UK, allowing populations to exist within urban areas as well as rural regions. The generation time is approximately two years, and adult breeding site fidelity between seasons is high (Blab, 1986). Common frogs spawn once a year, usually in the spring, and as each female usually lays only one clump of spawn each year, the number of clumps of spawn gives a reliable estimate of the number of reproductive females (Savage, 1961). By sampling a single egg from each clutch, it is theoretically possible to sample the entire reproductive output for a single year, thus providing an excellent basis for population genetics studies. Genetic diversity in the common frog is high (Reh and Seitz, 1990), enabling good resolution when investigating the genetic composition of different populations. 


\section{Amphibian disease}

Amphibians are currently undergoing worldwide declines (Houlahan et al, 2000); one third of amphibians are vulnerable, endangered or critically endangered according to the Global Amphibian Assessment led by the International Union for Conservation Biology (IUCN, 2004). The biggest threat to amphibian populations is habitat loss and degradation, which currently affect approximately 4,000 species. However the rate of declines caused by habitat loss is relatively slow and there are several strategies (such as the creation of protected areas) to control this threat (IUCN, 2004). Disease is not the most significant threat, but its effect is much more rapid and can cause sudden population declines and extinctions (IUCN, 2004).

Unusual and often large-scale mortalities have occurred in common frogs in the UK since approximately 1985 . These deaths are now known to be caused by a virus belonging to the genus Ranavirus (family Iridoviridae), which was likely introduced into the UK from North America in the 1980s (Cunningham et al, 1996). These mortalities can happen just once, causing one population crash, or multiple times, and may be reflected in the genetic variation of infected compared to non-infected populations. Today the geographic distribution of virus-related deaths has expanded from the SouthEast of England to encompass most of Britain, excluding Ireland (Cunningham et al, 1996). Very few cases of disease emergence are so well documented in terms of the pattern of emergence and subsequent spread over two decades. The long-term effect of declines caused by amphibian diseases is as yet unknown, and so studies of disease in a tractable amphibian species such as Rana temporaria are of particular importance.

\section{Historical and recent crises}

\section{Glaciation and range shifts}

The range of the common frog currently encompasses most of Europe, western Siberia and parts of northern Kazakhstan (Gasc et al, 1997). During the repeated continental glaciations of the Pleistocene epoch (roughly every 100,000 years from 1.8 million to 
10,000 years before present) this range would have been severely fragmented and displaced southwards into warmer, non-glaciated areas. Populations isolated in different refugia would have diverged genetically over time. As the climate warmed, range expansions northwards became possible. With this expansion, founder events would have led to a loss in genetic variation (Nichols and Hewitt, 1994). This picture replaces previous orthodoxy, which suggested that expansions occurred from refugia and were the result of substantial genetic drift (e.g. Skellam, 1951; Van den Bosh et al, 1988). In the southern refugial areas, which may have been quite large and may have persisted through the climatic cycles, a greater proportion of the original genetic variation would have been maintained. This type of history explains the present-day pattern observed in many European temperate species in which there is greater genetic diversity in southern populations (Hewitt, 2000).

In order to study the origins of modern genetic variation, we would ideally know the genetic composition of ancestral populations; however it is rarely possible to obtain such samples. Only a few species have been preserved from ancient remains, from which it is possible to obtain temporal samples throughout pre-historical times (penguins (Pygoscelos adeliae), Lambert et al, 2002; bears (Ursus spelaeus), Orlando et al, 2002; and kiwi (Apteryx spp.) Shepherd and Lambert, 2008). However where such samples are not available, it is possible to make some inferences of historical events from the present day geographic distribution of genotypes in extant populations. In this thesis I use mitochondrial DNA (mtDNA) to assess the genetic composition of common frogs in the British Isles, and to reveal the probable origins of populations throughout Britain and Ireland. Microsatellites are also used to establish the extent of the effect that recolonisation events have had on the genetic variation of extant populations. Together, microsatellites and mtDNA markers are used to describe the impact of a major historical event on genetic patterns in common frogs.

\section{Infectious disease}

There are many different possible outcomes for a population when a disease emerges, 
ranging from little impact to extinction. If a pathogen is very virulent, transmission rates are high, and/or the host has no immunity, then the host could be eliminated at a population or species level. Examples include the extinction of a snail species (Partula turgida) in Polynesia due to a microsporidian parasite (Cunningham and Daszak, 1998) and the extinction of certain amphibian species such as the golden toad due to a fungal disease, Chytridiomycosis (Pounds et al, 2006). In many cases, species affected by severe disease outbreaks do not go extinct, and remnant populations persist with endemic infections, as can also happen with Chytridiomycosis in some amphibian species (Brunner et al, 2004; Retallick et al, 2004). If a pathogen and its host's immune system are capable of evolving at a similar rate, a co-evolutionary arms race can occur. For example, gene-for-gene interactions among loci in barley (host) and leaf scald (pathogen) result in adjustments over generations between host and pathogen populations (Allard, 1990). It appears that common frog populations show a range of responses to infection with Ranavirus. Some populations appear to experience localised extinction and do not re-establish, some populations persist despite annual recurrences of Ranavirus-related mortalities, and some populations appear to clear infection completely after the initial mortality event. Populations which persist may do so through a process of selection for genotypes that confer immunity, or the population may be maintained through dispersal from nearby ponds.

In this thesis I use microsatellite markers to compare populations which have persisted despite experiencing recurrent Ranavirus mortalities, with those that have never shown signs of infection. By comparing the genetic composition of populations that have been subject to repeated selection (over approximately five generations) with those that are likely not to have undergone selection, it is possible to assess the impact of these largescale mortalities on population genetic variation. However, neutral variation does not always correlate with the variation of genes that confer a selective advantage such as immunity (Merilä and Crnokrak, 2001). Markers that confer an adaptive trait can be used to establish whether a proportion of genetic variation in a population (or group of 
populations) is attributable to a selective pressure. In this thesis, I describe the development of an adaptive marker at the Major Histocompatibility Complex (MHC). This region appears to be functionally important, as the MHC class Ia locus has been linked to Ranavirus susceptibility in Xenopus (Flajnik et al, 1987; Gantress et al, 2003). Proteins encoded by the MHC class I locus form complexes with antigenic (viral) peptides and present these at the cell surface to the T-cell antigen receptors, so there is a direct connection between the coding at this locus and the immune capabilities of the host. In this thesis I look for signals of selection by comparing sequences from the antigen-binding region of MHC class I in populations with and without a history of disease. Together, microsatellites and MHC are used to investigate the impact of Ranavirus emergence on the genetic variation of the common frog.

\section{Theoretical considerations}

\section{The importance of neutrality}

Measuring the genetic variation at different genomic regions can give an indication of the variation inherent among populations due to their different demographic histories, as well as distinguishing variation that is caused by other factors such as recent selection due to an introduced disease. The genomic regions used to assess neutral variation should be truly neutral and so should not be affected directly by selective events such as disease outbreaks. Microsatellites are commonly used to measure neutral variation, however in practice it can be difficult to ascertain true neutrality. Microsatellites should not be under direct selection, however there are a few exceptions; for example certain loci have been shown to act as enhancer elements in gene expression, to have protein binding abilities or to have phenotypic effects on physiology and development (Kashi and Soller, 1999). In some studies certain microsatellite loci show unusually low or high levels of genetic diversity; a factor that may be attributable to natural selection acting on a nearby locus to which the microsatellite is linked ('genetic hitch-hiking', see Beaumont and Nichols, 1996; Maynard Smith and Haigh, 1974; Watts et al, 2008). Selective sweeps occur when the genetic variation of regions flanking a gene (or genes) 
that is under selection is altered. Large regions around loci that are under selection can show non-neutral patterns of genetic variation; for example, a study on sticklebacks showed that a region of about $90 \mathrm{~kb}$ either side of a locus that was under selection was not neutral, providing good evidence for a selective sweep (Hannu et al, 2008). A similar effect to genetic hitch-hiking can occur when a neutral marker is linked to a deleterious allele which is selected against ('background selection', see Charlesworth et al, 1993).

As both hitch-hiking and background selection can result in a loss of genetic variation, they may interfere with detecting the effects of bottlenecks on populations, which can also result in a loss of genetic variation. However, population bottlenecks should have genome-wide effects detectable across all loci whereas hitch-hiking and background selection are unlikely to affect all loci. In order to differentiate between single locus and genome wide effects, it is important to use a large number of neutral loci, and to look for signals of selection in the individual loci used. For species where the genome has been sequenced it is relatively straightforward to identify whether microsatellites are likely to be linked to the area of the genome which is under selection. The common frog genome has not been sequenced or mapped, and so other methods must be considered for identifying loci which may be non-neutral. One such method is to consider the relationship between $\mathrm{F}_{\mathrm{ST}}$ and heterozygosity at each locus. Loci which show unusually high or low differentiation relative to heterozygosity may be linked to a locus which confers a selective advantage or disadvantage (Beaumont and Nichols, 1996).

Types of selection

Broadly speaking, selection either favours specific alleles or genotypes (for example a disease resistance allele), or favours having a mix of alleles (within an individual) or genotypes (within a population i.e. group selection, which is currently thought to be a side-effect of individual-level selection, see Koeslag, 2003). Directional selection occurs if an allele confers a selective advantage or disadvantage; alleles that are advantageous 
increase in frequency (positive selection) or alleles that are disadvantageous decrease in frequency (negative or purifying selection). In a simple case, if an additive allele $A$ provides resistance to a disease, we would expect to see the following fitnesses under selection from that disease: $w_{A A}>w_{A a}>w_{a a}$, where $w_{i j}$ indicates the fitness of the genotype $i j$. This form of selection would eliminate the $a$ allele and thus lead to an overall loss of genetic variation. For example, two Human Leukocyte Antigen (HLA - the name given to the human MHC) types associated with protection from malaria are common in West Africans but rare in other racial groups (Hill et al, 1991).

Balancing selection differs from directional selection in that balancing selection maintains genetic variation in a population. Three forms of balancing selection are heterozygote advantage, negative frequency-dependent selection and fluctuating selection. Heterozygote advantage (overdominance) occurs if the heterozygote has a fitness advantage over all homozygotes. The most well known example of heterozygote advantage is the case of malarial resistance in people that possess one sickle cell anaemia allele and one unaffected allele (Aidoo et al, 2002). Some MHC genes are thought to be under overdominant selection, as the presence of two alleles allows the recognition of a greater range of antigens (Hughes and Nei, 1988). The second form of balancing selection, negative frequency-dependent selection, occurs when an allele is more advantageous if it is rare. This form of selection has also been proposed as a model for MHC (e.g. Bodmer, 1972); this model is based on the concept that individuals with a recent mutant allele have a selective advantage over pathogens. In this case alleles, or allelic frequencies, could be expected to constantly change to keep ahead of pathogen evolution. For example rare HLA super-types can be advantageous in humans infected with HIV, a particularly fast-evolving pathogen (Trachtenberg et al, 2003). Finally, fluctuating selection occurs when a heterogeneous environment causes selection for different alleles over time and/or space (Hedrick, 2002). There is evidence that temporal fluctuating selection is a cause of polymorphism in TAP genes (Transporter associated with Antigen Processing, a gene with a role related to that of the MHC) in Danish brown 
trout (Jensen et al, 2008). Fluctuating selection could occur in a disease scenario if the pathogen is fast evolving, or if different strains occur in different populations. Detecting any type of selection is not straightforward, as various factors can alter the outcome of selection in natural populations including:

- $\quad$ The genetic composition of a population before selection occurs.

- $\quad$ The strength and type of selection.

- $\quad$ The time since selection occurred, and the length of time that it occurred.

\section{Linkage of selected loci}

Earlier I described the effects that linkage can have on neutral markers, and how this can complicate inferences made from changes in genetic variation. However, linkage is also relevant when investigating adaptive loci, as linkage can also occur between sites that are under selection. Linkage can slow down the response to selection and reduce its effectiveness, since selection can no longer act independently on any one of the linked loci (the 'Hill-Robertson effect', see Hill and Robertson, 1966). This effect adds to those already mentioned, which include hitch-hiking and background selelection. The allele frequencies change as if the effective population size were smaller: genetic drift is accentuated and hence genetic variation tends to be lost faster. Rates of recombination therefore play a part in determining genetic variability, for example in genomic regions where recombination is limited (e.g. near telomeres or centromeres), the Hill-Robertson effects may be more apparent. This issue is potentially of importance when studying MHC loci, as the MHC consists of multiple loci with different selective pressures acting upon them. Whilst the MHC class I is mostly responsible for the presentation of endogenous antigens such as viruses, the MHC class II loci are mainly responsible for the presentation of exogenous antigens such as bacteria. If class I and II are linked, selection at class II may influence the signal of selection seen at class I, although the presence of introns between MHC loci may increase recombination and so reduce this effect (Comeron et al, 2008). The design of the study presented in this thesis should avoid problems caused by the Hill-Robertson effect. Populations with and without 
Ranavirus infection are interspersed within the same geographical region, with similar spread, reducing the likelihood of another selective event (e.g. a different pathogen) occurring by chance in one group but not the other.

\section{Outline of the thesis}

The second and third chapters consider the impacts of major historical glaciation events on the genetic variability of common frog populations in the British Isles. The rest of the thesis focuses on the recent impacts of Ranavirus on common frogs in England. Chapter 4 describes how Ranavirus outbreaks were detected, how study sites were chosen and the impact of the disease on population size. The fifth and sixth chapters assess the impact of Ranavirus on the genetic variation of common frog populations. Each chapter contains an introduction, methodology, analysis and discussion specific to the questions addressed.

\section{Chapter 2. Phylogeography of the common frog}

To establish an evolutionary context, Irish and British common frogs are considered within the context of European glacial history. The British Isles were mostly covered by ice during the Pleistocene glaciations resulting in a loss of habitable area for temperate species. There are a variety of suggested routes of recolonisation into Britain and Ireland following the glaciations. Sequencing of the mitochondrial cytochrome $b$ gene from samples throughout Europe allows comparisons between haplotypes found in different areas. The probable recolonisation route taken by the common frog into Britain is established. Support is assessed for the three most likely recolonisation routes into Ireland: Re-introduction by humans from Iberia; species moving across a land bridge from Britain; and species surviving the glaciations in a small refugium in the south of Ireland.

\section{Chapter 3. Modern genetic variation in England and Ireland}

In order to investigate whether the glaciations have also affected the genetic diversity of contemporary common frog populations in the British Isles, the within and between 
population genetic variation of a subsection of Irish and British disease-free common frogs are compared. It is likely that Ireland was in part colonised through Britain, and as such, Irish frogs might be expected to show reduced genetic variation consistent with founder events. The null hypothesis that the genetic structure of Irish populations is the same as that of British populations is tested. Neutral genetic variability is assessed in Irish and UK populations using microsatellite markers to measure allelic richness, heterozygosity and population differentiation.

\section{Chapter 4. The impact of Ranavirus on common frogs}

Many common frog populations in the UK (but not Ireland) have been infected with Ranavirus. As this disease causes mass mortality events, it is possible that it could have a longer-term affect on population size, and potentially alter the genetic patterns seen in UK common frog populations. This chapter sets out the methods for determining whether a frog population is or has been infected with Ranavirus. Signs of infection are discussed together with the analysis of public reports of frog mortalities and the rationale behind the selection of populations used. Direct evidence of current infection is assessed from the presence or absence of Ranavirus within frog tissues. Public reports of mass mortalities are used to determine the average number of frogs killed in a Ranavirus mass mortality event. I follow up archived records from 1996-2008 to assess whether populations with a history of recurrent disease have undergone population size reductions.

\section{Chapter 5. Neutral diversity and disease}

To investigate whether population genetic structure is influenced by viral emergence, I use microsatellite markers to compare genetic variation in British common frog populations with and without Ranavirus infection. Microsatellite loci are checked for signals of selection that might indicate that the markers are not truly neutral. The effects of disease outbreaks on genetic variation are established, based on the null hypothesis that populations with and without disease will show the same levels of within and 
between population diversity. Simulations of the effects of population bottlenecks and different mating systems on genetic diversity are run in order to help verify the findings. Chapter 6. Adaptive diversity and disease

Microsatellite markers are not expected to play an adaptive role under selection, so looking at how an adaptive marker differs with infection status may give a more complete picture of how Ranavirus affects common frog populations. In this chapter, I describe the development of a marker to study adaptive diversity at the Major Histocompatibility Complex (MHC) Class I locus; a gene involved in the immune system and thought to affect resistance to Ranavirus infection in Xenopus. The locus is used to investigate whether a signal of selection can be detected in infected populations. MHC sequences are analysed to identify whether certain supertypes are associated with the infection status of the population. MHC diversity in populations with and without disease is estimated and the results are compared with those from microsatellite markers.

Finally, in chapter 7, I summarise the main findings of the thesis and present some general conclusions in the broader context of population genetic theory. I also discuss the more practical conservation and management implications that can be drawn from the results. 


\title{
CHAPTER 2: European phylogeography of the common frog (Rana temporaria), and postglacial colonisation routes into the British Isles.
}

Accepted (October 2008) for publication as:

Teacher, A.G.F., Garner, T.W.J., Nichols, R.A. European phylogeography of the common frog (Rana temporaria): routes of postglacial colonisation into the British Isles, and evidence for an Irish glacial refugium. Heredity

\begin{abstract}
In this chapter, I used phylogenetic techniques to investigate the postglacial repopulation of Europe by the common frog and, in particular, the colonisation of Ireland. Three main hypotheses have been proposed for the re-establishment of the Irish fauna after the last ice-age: arrival across a late-glacial land bridge from Britain; expansion from a glacial refugium in the South of Ireland and, for some species, re-introduction by humans from Iberia. I examined the phylogeographic structure of 52 populations of the common frog (Rana temporaria) throughout Europe using 476 base pair mitochondrial cytochrome $b$ gene sequences. These data replicate previous studies in showing substantial sequence divergence (3\%) between Eastern and Western European common frog haplotypes. However, I uncover new evidence that these haplotypes co-exist in Spain, Switzerland and France, and infer an expansion of the Eastern clade along the Mediterranean coastal corridor. All the British samples fall within the Western European clade, but the Irish data imply a different history. Genetically distinct haplotypes occur in populations from the South-West of Ireland. This local genetic differentiation may be a consequence of a local glacial refugia, possibly combined with natural colonisation or introduction from Western Europe.
\end{abstract}




\section{Introduction}

The earth's climate appears to have warmed and cooled in cycles of approximately 100,000 years, with the last ice sheet retreating approximately 10,000 years ago (Webb and Bartlein, 1992). These glaciations had profound effects on temperate species distributions, causing severe fragmentation of populations. In Europe most temperate species were displaced southwards towards non-glaciated refugia. After glaciation, species expanded northwards to occupy territories with improved climates and habitats, losing genetic variation along the way by founder events (Nichols and Hewitt, 1994). Isolation in refugia and the subsequent recolonisation events allowed populations to diverge genetically. These events shaped the genetics of many species so fundamentally that geographical differences can still be detected in contemporary populations, and colonisation history can be reconstructed (Hewitt, 2000).

Although still debated, the majority of evidence supports a general pattern of Southern European refugia where temperate fauna survived, and North-Eastern European refugia where boreal or cold-temperate species survived (Hewitt, 2004; Malez, 1972; Taberlet et al, 1998). A study of ten temperate taxa (including five amphibians; Triturus spp.), revealed similarities in their reconstructed colonisation routes, with Northern Europe being colonised via three main routes, from Iberian, Italian and Balkan refugia (Taberlet et al, 1998). However, as well as these large refugia, some authors argue that there were many small Pleistocene refugia in central and Northern Europe; such refugia would have been in sheltered regions with relatively stable microclimates, and have been identified in Norway, Belgium, the UK, Hungary, Ireland and Slovakia (for a review, see Steward and Lister, 2001).

Despite similarities in the broader patterns of colonisation, differences have been inferred in the history of colonisation of different species (Taberlet et al, 1998). For example a thorough study of the white oak (Quercus spp.) using chloroplast DNA variation concluded that these trees appear to have colonised Britain via France having followed a route from a refugium in Iberia (Dumolin-Lapegue et al, 1997). Studies on 
pool frogs (Rana lessonae) show an interesting alternative route into Britain via Poland from a refugium in Italy, based on microsatellite and RAPD analyses (Snell et al, 2005; Zeisset and Beebee, 2001). These distinct histories might be explained by differences in the ecology of the species, differences in the refugial origins of the colonisers, or by events affecting the rate of expansion from different refugia. The postglacial colonisation of Ireland is particularly interesting due to the wealth of conflicting evidence. There are three main hypotheses explaining the postglacial re-establishment of the Irish fauna, which are not mutually exclusive: a species may have arrived by immigration across a land bridge from Britain, it could have survived throughout the glaciations in a refugium in the South of Ireland, and then expanded, or it could have been translocated by humans.

Some authors have argued that a late-glacial land bridge existed between Ireland and Britain, based on sea-level modelling (Lambeck, 1996; Lambeck and Purcell, 2001; Wingfield, 1995) and biostratigraphic evidence (Preece et al, 1986). Although there is limited evidence for species colonising Ireland via a land bridge, existing evidence comes from the fossil remains of the mountain hare (Lepus timidus) and the stoat (Mustela erminea) found in Ireland. The samples date to the late glacial period before humans arrived in Ireland, ruling out human introduction (McCormick, 1999; Woodman et al, 1997). However, these data could be re-interpreted not as evidence of colonisation via a land-bridge but as the animals having survived the glaciations within Ireland, as both species are cold-tolerant (Stuart and Van Wijngaarden-Bakker, 1985). The position (and existence) of any land bridge remains unresolved, with suggestions that it spanned from Scotland (Devoy, 1985) or from France via the South-West of Britain (Lambeck, 1996; Lambeck and Purcell, 2001). Under the land-bridge hypothesis we would expect to see British and Irish samples occurring within the same monophyletic group. However, high divergence between British and Irish populations has been found in certain species. For example Hamill et al. (2006) found that populations of mountain hares (Lepus timidus) in Scotland and Ireland belonged to different mtDNA clades and 
showed high divergence based on nuclear microsatellite data, reporting $\mathrm{F}_{\mathrm{ST}}$ values of over 0.4 .

Another possible colonisation route into Ireland is anthropogenic introduction. Some evidence suggests humans could have introduced species from Iberia, as trade routes are thought to have existed between Ireland and Iberia over 2000 years Before Present (Corbet, 1961; Corbet, 1962; O'Rourke, 1970; Praeger, 1939). Several species are either found only in South-West Ireland and in Iberia, or have very different distributions in the geographically intermediate countries (e.g. the pygmy shrew (Sorex minutus), Mascheretti et al, 2003; the strawberry tree (Arbutus unedo), Mitchell, 1986; and the Kerry slug (Geomaculosus maculosus), Platts and Speight, 1988). Under this hypothesis we would expect to see Irish and Iberian species falling within the same monophyletic group.

An alternative explanation for divergence between British and Irish populations is that of a refugium in Ireland. The Pleistocene ice sheets may not have covered the whole of Ireland and there is evidence that the South-West of the island remained an ice-free area of open steppe tundra (Forbes, 1846; Yalden, 1999). This ice-free region is hypothesised to have acted as a refugium in which species could have survived the glaciations (Forbes, 1846; Hoarau et al, 2007). Under this hypothesis we might expect to find unique haplotypes in Irish populations, which are divergent from those found elsewhere in Europe, forming a distinct monophyletic group. Microsatellite and mtDNA evidence from Natterjack toads (Bufo calamita) indicate higher divergence times between Northern England and Ireland compared to Southern England and Ireland (Rowe et al, 2006). This finding led Rowe et al. (2006) to propose a source population between South-West England and Ireland, an area which would have been partly dry land at the time (approximately 11,000 years BP) (Devoy, 1985). 


\section{The study organism}

The common frog, Rana temporaria, inhabits the United Kingdom and Ireland and is widespread throughout Europe (Fig. 2). This species provides a good model system for examining postglacial colonisation as it is widespread throughout Europe, and has the greatest genetic variability of all western Palearctic brown frogs (Reh and Seitz, 1990). Common frogs can survive in very cold environments, and can be found at high altitudes; the highest recorded are in the French and Swiss Alps at approximately 2,630m (Gasc et al, 1997). Palo et al. (2004) surveyed European populations of common frogs and carried out analysis of mitochondrial cytochrome $b$ sequences and variation in allele frequency at nuclear microsatellite loci. They found evidence for separate Western and Eastern lineages; the differences in DNA sequences were interpreted to mean that they shared a common ancestor approximately 700,000 years ago, coinciding with the onset of Pleistocene glaciation. However, common frog populations have not been well studied in the British Isles, and could shed light on how these islands were colonised following the glacial periods. This study provides additional phylogeographic coverage of Europe, with particular reference to assessing evidence for the various potential colonisation routes into Britain and Ireland. To approach these questions, I examine variation in mitochondrial DNA (mtDNA) cytochrome $b$ (Cyt $b$ ) gene sequences in populations of $R$. temporaria throughout Europe, with focussed sampling in Ireland. Mitochondrial DNA can be used to infer population histories dating back to the Pleistocene as the mutation rate is rapid enough to create variability whilst the absence of recombination makes the inference of relationships between haplotypes straightforward (Avise, 2000).

\section{Materials and Methods}

I collected samples (Rana temporaria spawn) from the UK and the Republic of Ireland, and other samples were donated from colleagues in Russia, France, Switzerland, Poland, Spain, Denmark, Italy, Finland, Germany, Sweden and Austria (Fig. 1). Samples from Sweden, Denmark and Finland were extracted using a standard salt extraction method (Aljanabi and Martinez, 1997) whilst those from Italy were extracted using the protocol 
as described by Boyle et al. (2004). All other samples were extracted using the Wizard SV96 Genomic Purification System (Promega, UK). In total, 131 individuals from 52 locations ( $\mathrm{n}=1$ to 6 per location) were used for mtDNA sequencing and analysis.

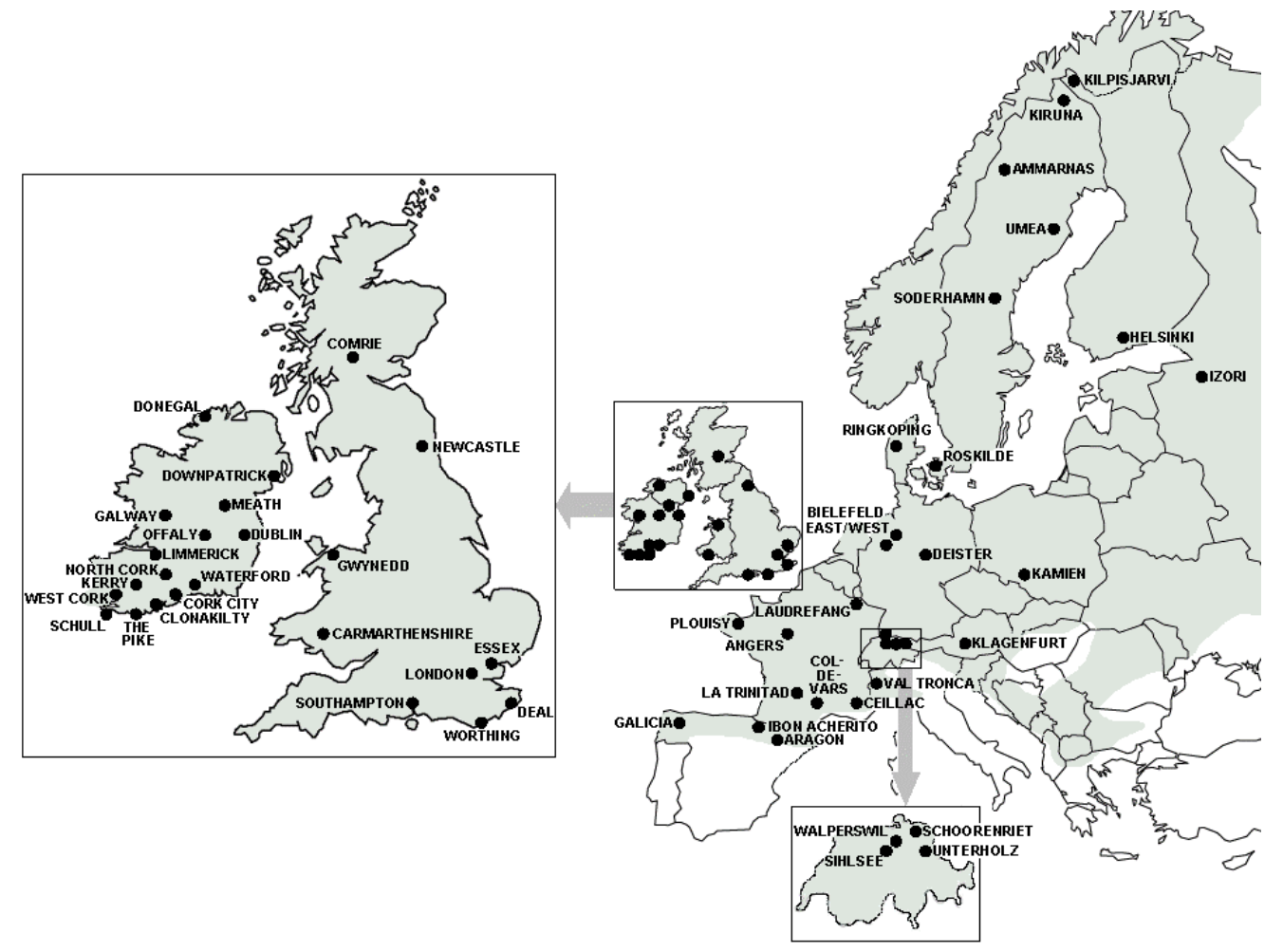

Figure 1. Map of 52 sampled locations in Europe. The approximate range of Rana temporaria is shaded in grey (Gasc et al, 1997).

DNA was amplified using polymerase chain reaction (PCR) which was performed using primers L14850 (5' '-TCTCATCCTGATGAAACTTTGGCTC-3' (Tanaka et al, 1994) and H15410 (5'-GTCTTTGTAGGAGAAGTATGG-3' (Tanaka et al, 1996) which amplify a 605-base-pair Cytb segment (Palo et al, 2004). Reactions consisted of $8 \mu 1$ Qiagen Taq PCR Master Mix (Qiagen, UK), $1 \mu \mathrm{l}$ of each primer at $100 \mathrm{pmol} / \mu 1,8 \mu \mathrm{l}$ of autoclaved and double-distilled water and $4 \mu 1$ extracted DNA. The PCR program was 12 minutes denaturation at $95^{\circ} \mathrm{C}$ followed by 25 cycles of denaturation at $94^{\circ} \mathrm{C}$ for $30 \mathrm{sec}$, annealing at $50^{\circ} \mathrm{C}$ for $40 \mathrm{sec}$ and extension at $72^{\circ} \mathrm{C}$ for $40 \mathrm{sec}$, followed by a final extension phase at $72^{\circ} \mathrm{C}$ for 7 minutes. PCR products were run on a $1.2 \%$ agarose gel using $5 \mu 1$ PCR product with $3 \mu 1$ loading buffer, and an 100 base-pair ladder (Microzone 
Ltd., UK). When the PCR produced a clear band of the expected size, the band was cut from the gel, and extracted using the QIAquick Gel Extraction Kit (Qiagen, UK). Gel extracted samples were used for direct sequencing. For sequencing, the reagents were: $5 \mu 1$ Better Buffer (Microzone Ltd., UK), $1 \mu 1$ BigDye Terminator 3.1 (Applied Biosystems, UK), $1.5 \mu 1$ primer at $16 \mathrm{pmol} / \mu 1,4.5 \mu 1$ autoclaved double-distilled water and $3 \mu 1$ purified PCR product making a total reaction volume of $15 \mu 1$; one reaction was performed using each of the two original primers in order to obtain overlapping forward and reverse sequences. The sequencing reaction PCR program specified 3 minutes denaturation at $96^{\circ} \mathrm{C}$ followed by 25 cycles of denaturation at $96^{\circ} \mathrm{C}$ for $15 \mathrm{sec}$, annealing at $50^{\circ} \mathrm{C}$ for $10 \mathrm{sec}$ and extension at $60^{\circ} \mathrm{C}$ for 40 minutes. The $15 \mu 1$ sequencing reaction products were then cleaned to remove unbound terminators using an EDTA/ethanol based protocol (see Appendix 1). Cleaned products were denatured at $95^{\circ} \mathrm{C}$ for 2 minutes followed by snap cool prior to automated sequencing on an ABI 3100 Genetic Analyzer (Applied Biosystems).

Sequences were aligned using Sequencher v.4.8 (Gene Codes Corporation), and clean sequence was obtained for 476 base pairs for all samples. For locations where multiple samples were of the same haplotype, a consensus sequence was produced for each haplotype present. From these condensed data (GenBank accession numbers FJ030808FJ030872), polymorphic sites and haplotypes were identified using TCS v.1.21 (Clement et al, 2000). Modeltest (Posada and Crandall, 1998) was used to test 56 possible DNA substitution model parameters. The best fit model was used to generate a maximum likelihood phylogenetic tree using Phyml Online (Guindon et al, 2005), with 100 bootstrap replicates to assess node support. Bayesian analysis was performed using MrBayes (Huelsenbeck and Ronquist, 2001) with random start trees, sampling every 100 generations for $5 \times 10^{5}$ generations using the HKY model of evolution. Neighbour Joining and Maximum Parsimony trees were constructed using Phylip programs (Felsenstein, 1989) with 100 bootstrap replicates. Trees were constructed using a published Rana arvalis Cytb sequence (GenBank accession number AY156954, Palo and Merilä, 2003) as an outgroup. Multiple phylogenetic methods were used to allow for the collation of 
consistent results and the avoidance of artefactual results (as per Holder and Lewis, 2003).

\section{Results}

Eighteen haplotypes were identified from the 131 sequences analysed (see Appendix 2). More than one sequence was obtained for 40 of the 52 locations, often with identical haplotypes (see Fig. 1). I identified 26 polymorphic sites, four with transversions, and no insertions or deletions. Modeltest (Posada and Crandall, 1998) determined that the least complex model of evolution with the best fit to the data under the hierarchical likelihood ratio tests was the $\mathrm{HKY}+\mathrm{G}$ model which allows for different transition and transversion rates and different rates of evolution at each nucleotide position (Hasegawa et al, 1985). The parameters of the model were: base frequencies: $A=0.2370, C=0.2770, G=0.1644$, $\mathrm{T}=0.3216 ; \mathrm{t} / \mathrm{t} v \mathrm{vatio}=8.2987$; and a Gamma distribution shape parameter of 0.1991 to describe the among-site rate variation at variable sites.

Bayesian, maximum likelihood, maximum parsimony and neighbour joining trees all showed support for the same major clades with few minor variations (Fig. 2). Two deep lineages were indicated, separated by mutations at 12 nucleotide positions. One lineage was found predominantly in Eastern Europe (to the East of France and Germany) and the other in the West. The extended sampling also revealed a previously undescribed distribution of the Eastern clade along the French Mediterranean coast and into Spain (Fig. 3). English and Welsh samples clustered in the main western clade, together with one Scottish sample. The majority of the Scottish samples clustered with Spain and one South-Eastern French sample in a western sub-clade. Samples from throughout Ireland grouped with the main western clade however, two Irish sub-clades diverged from the western clade and consisted almost exclusively of Southern Irish samples (Fig. 2). One Irish sub-clade (haplotypes 13-15) showed a unique change in one amino acid from alanine to valine. 


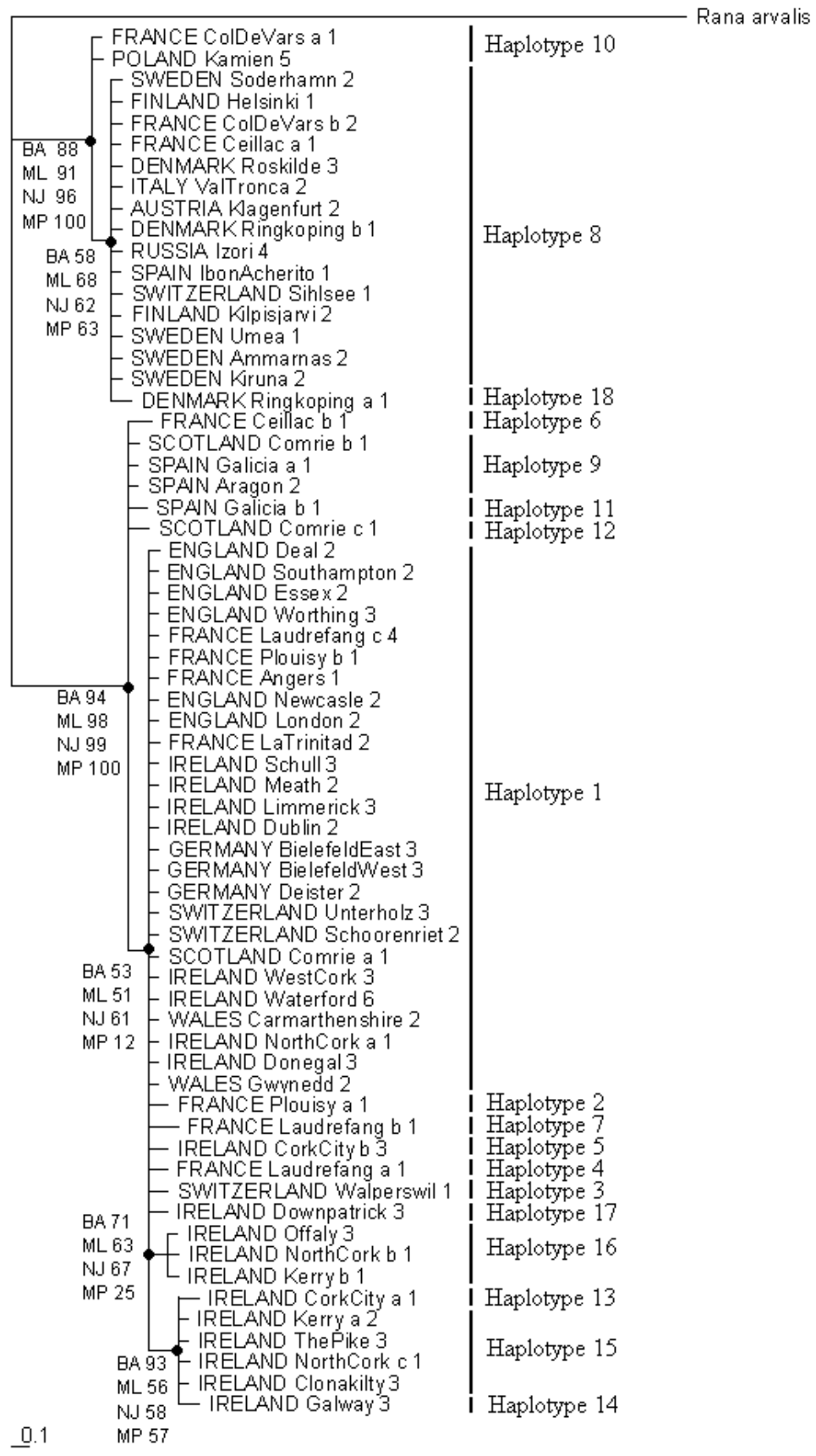

Figure 2. Phylogenetic tree. Bayesian tree based on 476 base-pair Cytb sequences with haplotypes marked. Support values are represented at major nodes for all statistical methods of tree-building used ( $\mathrm{BA}=$ Bayesian, $\mathrm{ML}=$ Maximum likelihood, $\mathrm{NJ}=$ Neighbour joining, $\mathrm{MP}=$ Maximum Parsimony). If more than one haplotype was found at a single location, the location name is followed by a letter $(\mathrm{a}, \mathrm{b}, \mathrm{c})$.

Following each location name is a number which represents the number of sequences obtained of the same haplotype at the same location. 


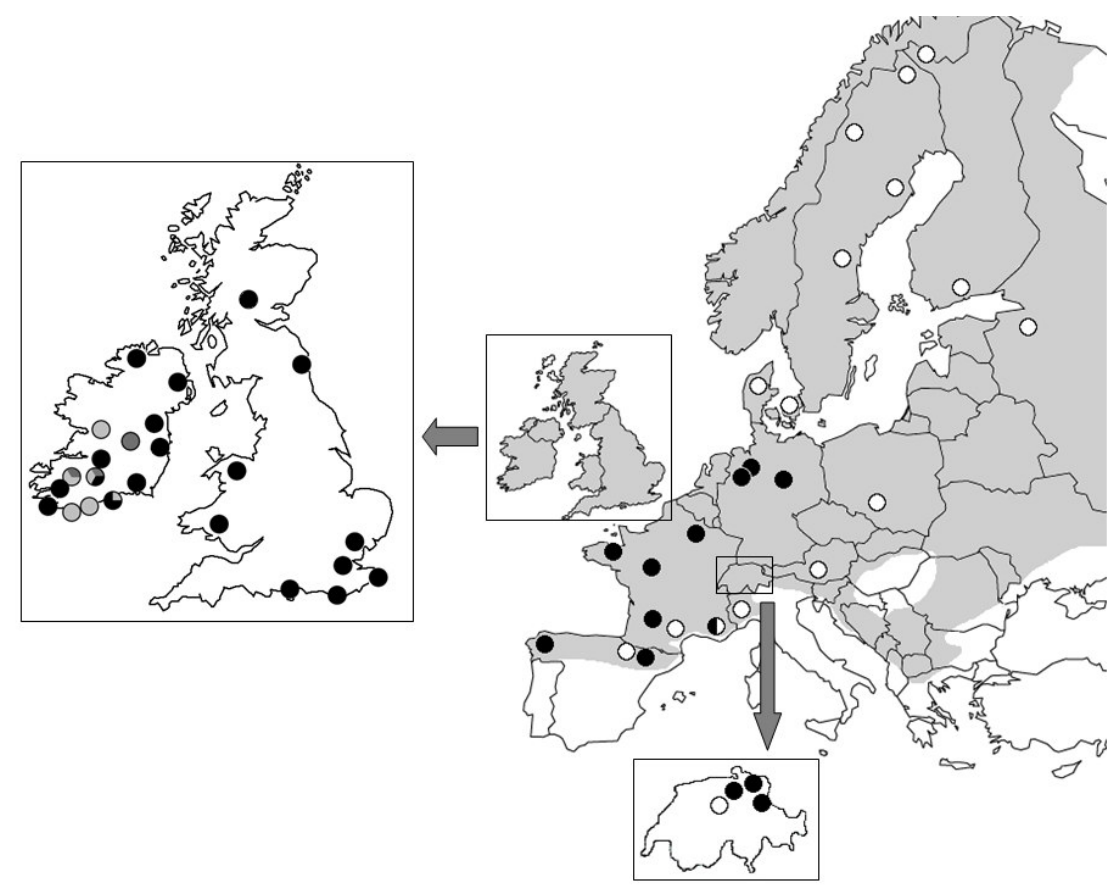

Figure 3. Haplotype distribution in Europe. Map to show the distribution of European clades. The Eastern clade (haplotypes $8,10,18$ ) is shown by white circles, and the main western clade (haplotypes 1$7,9,11,12,17)$ is shown by black circles. The approximate range of Rana temporaria is shaded in grey (Gasc et al, 1997). On the inset map of the British Isles, Irish sub-clade one (haplotypes 13-15) is shown in pale grey, and Irish haplotype 16 is shown in dark grey.

\section{Discussion}

A split within Europe

Expansion from different glacial refugia has affected the current distributions of genetic lineages. Northern Europe was colonised from three main refugia, in Iberia, the Balkans, and Italy (Taberlet et al, 1998). Italian lineages of some taxa (for example, the grasshopper Chorthippus parallelus) did not colonise Northern Europe as the Alps acted as a barrier (Taberlet et al, 1998). A substantial split between eastern and western mtDNA sequences in the common frog was identified by Palo et al. (2004) and is replicated in this study. However, my data demonstrate an overlap between the eastern and western lineages not found previously. The eastern clade penetrates along the French Mediterranean coast and over the Pyrenees into Spain. The clades co-occurred in the Ceillac population (France, Figs. 1 and 2); in this population the western clade is 
represented by a unique haplotype (haplotype 6) which differs from the main western clade (haplotype 1) by three nucleotide polymorphisms, two of which are identical to those found the main eastern haplotype (haplotype 8). It is possible that this represents recombination between the two lineages (Slate and Gemmell, 2004), though it is more likely to either be caused by homoplasy, or the retention of an ancestral state which has been lost in other western haplotypes. It would be interesting to perform intensive sampling along the contact zone between Eastern and Western haplotypes to further investigate this occurrence.

North-Eastern Spanish, South-Eastern French, Northern Italian, one Swiss and all Austrian samples group with Fennoscandian samples in the eastern clade. Regardless of the direction of colonisation, the route linking these populations follows a contour around the major mountain ranges of the French and Italian Alps and the Pyrenees. Although the Alps appear to have acted as a barrier to some species (including newts, Triturus carnifex, Taberlet et al, 1998), common frogs survive at very high altitudes in these mountains (Gasc et al, 1997). The sampling locations in my study in the Italian Alps (Val Troncea) and French Alps (Ceillac) imply that the Alps form a permeable barrier to the common frog, restricting rather than preventing gene flow. Nevertheless, the distribution of the Eastern clade along the Mediterranean coast suggests that the mountains may have channelled colonising founders. Ancestors of the Western clade most likely originated in an Iberian refugium, colonising North-Western Europe as the climate warmed. The distribution of the Eastern clade indicates that the refugial populations could have been situated in Italy or the Balkans, with expansion both into North-Eastern Europe and to the West along the Mediterranean coast (Fig. 2).

Previous studies on other amphibian species in Eastern Europe have implied multiple refugia in the East. For example, a study on mtDNA haplotypes of fire-bellied toads (Bombina bombina and Bombina variegata) in South-Eastern Europe showed that there were three distinct clades, and suggested refugia in the Carpathians and Apennines as well as the Balkans (Hofman et al, 2007). A study on mtDNA haplotypes in the moor 
frog (Rana arvalis) indicated that multiple lineages co-exist in Eastern Europe, and may originate from refugia in the Carpathians and Southern Russia (Babik et al, 2004). In my study, the Eastern clade provides no indication of multiple refugia in this region, however the sampling range did not extend as far East as these other studies; sampling further into Russia and Northern Kazakhstan could prove fruitful for assessing the existence of additional refugia in this area.

\section{The colonisation of Britain}

Zeisset and Beebee (2001) provided evidence for the colonisation of pool frogs (Rana lessonae) into Britain from Poland via an Eastern route originating in Italy; microsatellite data (6 loci) grouped British samples into a distinct northern clade with Norway and Sweden. The data from this study provide no support for this hypothesis in the common frog, as British frogs fall within the western clade whilst Polish and Scandinavian frogs are in the eastern clade. All samples from England and Wales are representative of the main western clade, and so colonisation of these areas is likely to have occurred via France or nearby surrounding regions. However, it is possible that microsatellites (as analysed for $R$. lessonae) and mtDNA may give different results for the same populations; microsatellite markers are faster evolving, and so are better suited to provide information on fine-scale and/or contemporary distributions and genetic diversity (e.g. Bowcock et al, 1984).

Three samples were obtained from the same location in Scotland, comprised of three distinct haplotypes. Two of these Scottish haplotypes group closely with Spanish samples. One speculative explanation is that they provide evidence of an anthropogenic translocation from Iberia. If such an introduction has occurred, it is likely to be recent or isolated as these haplotypes are not found elsewhere in the British Isles. However, a more parsimonious explanation might be that of retained ancestral polymorphism, or of homoplasy. It is not possible to distinguish between these possible explanations using my data; however additional molecular markers and sampling within Scotland could provide an answer in the future. 


\section{The colonisation of Ireland}

The haplotypes found in Ireland are particularly interesting, as some form monophyletic groups that are unique to Ireland whilst others group within the main Western European clade. I propose two hypotheses to explain this pattern: (1) Irish frogs survived in a refugium in Ireland where some acquired novel mutations and others retained their ancestral state (2) A dual colonisation occurred where some frogs survived in a refugium in Ireland (the Irish haplotypes), and some frogs colonised via a land bridge or were introduced from Western Europe (the ancestral haplotypes). Interestingly, there is a historical reference to a Fellow of Trinity College Dublin moving common frogs from England to Dublin in 1696, and others have hypothesised that this was the route of colonisation (Smith, 1964). An alternative hypothesis - that of an anthropogenic introduction to Ireland from Iberia, gains no support from this study, as populations in Ireland show no specific similarity to those in Spain.

\section{Conclusions}

Post-glacially established common frogs (Rana temporaria) are divided into Western and Eastern European lineages. The ranges of these lineages overlap along the French Mediterranean coast and as far West as the Spanish side of the Pyrenees, and both lineages co-occur in at least one population in Southern France. Britain appears to be colonised from the western lineage, with a probable origin in an Iberian refugium. Ireland has haplotypes belonging to the western lineage as well as some unique mtDNA sequences that are consistent with survival in an ice-free refugium in Ireland. Thus, a dual colonisation of Ireland may have occurred, and contemporary Irish common frog populations may have ancestors both from a refugia within Ireland, and from the Western European lineage. The results of this study indicate that the postglacial colonisation of Europe by common frogs may be more complicated than has previously been assumed, as an additional potential refugium for this species has been identified. It is possible that more detailed sampling within Europe could reveal further small, cryptic refugia. 


\title{
CHAPTER 3: Microsatellite analysis reveals dramatic differences in population genetic variation of the common frog (Rana temporaria) in England and Ireland
}

\begin{abstract}
Phylogenetic studies have previously shown that common frog (Rana temporaria) mtDNA cytochrome $b$ haplotypes in the East of Ireland are indistinguishable from British, and most Western European haplotypes. As it is likely that Ireland was recolonised in part via Britain, evidence of founder events may be detectable in Irish populations. Theory predicts that recolonisation via founder events results in a loss of genetic diversity with distance from the source population. In this study I used 14 microsatellite markers to compare the genetic composition and diversity of common frog populations in the East of Ireland and in the South-East of Britain. A dramatic reduction in allelic richness and heterozygosity was identified in Irish compared to British populations. The majority of alleles present in Ireland represent a subset of the alleles in Britain, yet correspondence analysis indicates that Irish and British individuals cluster into distinct groups based on allelic frequencies. No differences in $\mathrm{F}_{\text {IS }}$ or $\mathrm{F}_{\mathrm{ST}}$ were detected between British and Irish populations, indicating similar population structuring. The findings demonstrate that phylogenetic studies using slow-evolving markers (such as mtDNA) can miss considerable differences in genetic composition and diversity between populations.
\end{abstract}




\section{Introduction}

European Pleistocene glaciations profoundly shaped modern species distributions, and the ancestry of individual populations can often be traced to a particular glacial refuge and colonisation route (Hewitt, 2000). Recolonisation after glaciation is likely to have taken place by a series of founder events, where new populations were begun from a few long-distance migrants, and expanded through localised spread (Nichols and Hewitt, 1994). The genetic diversity of founded populations is usually lower than that of the source population, due to the subsampling of individuals (Nei et al, 1975). This occurrence can help to explain the phenomenon whereby populations that are further from a refugial area often have reduced genetic diversity (Hewitt, 2000). This pattern has been observed in several amphibian species, for example the Italian agile frog, Rana latastei (Garner et al, 2004), and the golden-striped salamander, Chioglossa lusitanica (Alexandrino et al, 2001). The colonisation of islands can result in similar patterns, as these colonisation events are particularly likely to involve very few individuals due to less direct routes of expansion (Abdelkrim et al, 2005; Whitaker, 1998). Furthermore, the increased isolation of island populations, and the subsequent lack of gene flow, is expected to increase the genetic divergence from the source population (Whitaker, 1998). Populations with limited gene flow between them can show a signal of isolation by distance, where populations that are geographically further apart show greater genetic differentiation (Wright, 1943). Changes in allele frequency, including the loss or fixation of alleles, can also be caused by genetic drift, which acts in the direction of increasing divergence between isolated populations (Cohan, 1984), and impacts most strongly on small populations (see Frankham et al, 2002).

The previous chapter showed the existence of unique mitochondrial DNA (mtDNA) haplotypes of common frogs in the South-West of Ireland, and suggested that this local genetic differentiation may be a consequence of a glacial refuge located towards the South of Ireland. However, haplotypes from the East of Ireland (around Dublin) were found to be indistinguishable from those in England and the rest of Western Europe, 
indicating a possible secondary colonisation event from Western Europe. This colonisation may have been natural (via a putative land-bridge from Britain, see Lambeck, 1996; Lambeck and Purcell, 2001; Wingfield, 1995) or through a later anthropogenic introduction from Britain (Smith, 1964). Although mtDNA did not distinguish any differences between common frog populations in Britain and North/Eastern Ireland, theory suggests that if this species recolonised Ireland via England, there would have been a concurrent loss of genetic diversity, and an increase in divergence caused by low levels of subsequent gene flow. Mitochondrial DNA has a relatively low mutation rate and thus is relatively invariant within species; finer-scale patterns, and differences in genetic diversity may not be detectable (Goldstein et al, 1995). Microsatellites are considerably faster evolving, and have a much higher resolution than mtDNA over shorter evolutionary time spans and smaller geographic scales; subsequently they can offer an alternative picture of populations, providing information on fine-scale and/or contemporary distributions and genetic diversity (e.g. Bowcock et al, 1984). In this study, I use microsatellite markers to further investigate common frog populations in England and Eastern Ireland. I examine Eastern Irish populations for a decrease in genetic diversity compared to English populations, and look for a signal of divergence between the two islands.

\section{Methods}

\section{Sampling}

I collected samples from garden ponds in the Republic of Ireland $(n=14)$, within an $80 \mathrm{~km}$ radius of Dublin, and in England $(\mathrm{n}=16)$, within a 90km radius of London (Fig. 1). Differences in habitat fragmentation should not impact the results, as care was taken to ensure that samples were obtained from similar habitats by only sampling urban and suburban garden ponds. For each site, sampling was performed on a single day in the breeding season between February and March 2005. For all English sites and five Irish sites, one egg was sampled from each clutch of eggs present in each pond. Embryos were grown to Gosner stage 19-20 (Gosner, 1960), and dissected from the egg mass. The remaining nine Irish sites were sampled in a different manner, as the short breeding 
season meant that not all ponds could be visited while it was still possible to clearly identify egg clutches. At these sites, I sampled approximately forty tadpoles (Gosner stage 22-24) in April 2005. Tadpoles were euthanased using 5\% MS222 buffered with $\mathrm{NaHCO}_{3}$. The tail of each embryo or tadpole was used for DNA extraction with the Wizard SV96 Genomic Purification System (Promega, UK).

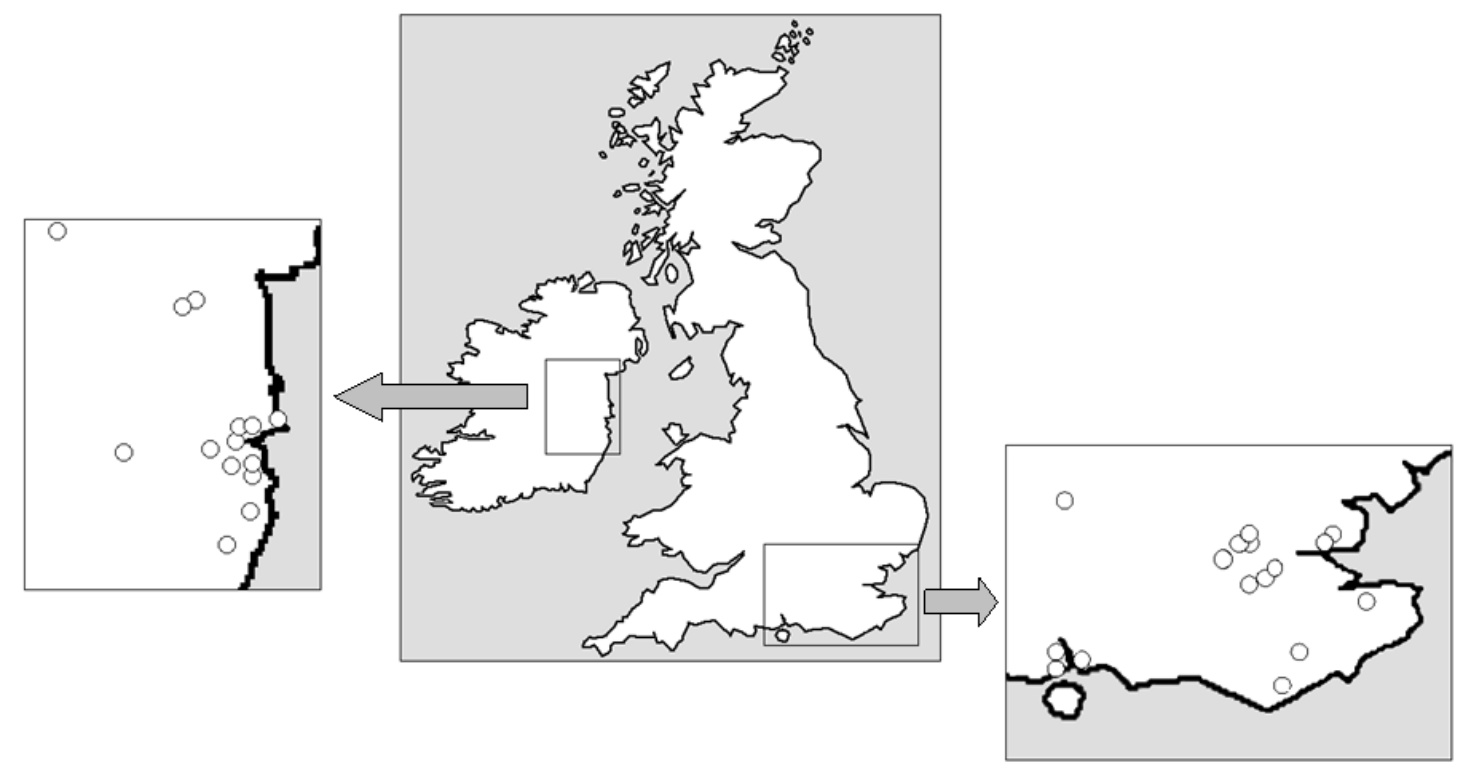

Figure 1. Map showing the location of populations sampled in England and Ireland.

\section{Microsatellite genotyping}

Fourteen microsatellite primer pairs were used (see Table 1 for primers and multiplex arrangements). PCR was performed using $4 \mu 1$ Qiagen Multiplex Mix (Qiagen, UK), $2 \mu 1$ template DNA, $2 \mu \mathrm{M}$ of each primer, made up to a total volume of $8 \mu \mathrm{l}$ with doubledistilled and autoclaved water. The PCR program used was: $95^{\circ} \mathrm{C}$ for 15 minutes, followed by 30 cycles of $94^{\circ} \mathrm{C}$ for $30 \mathrm{sec}, 57^{\circ} \mathrm{C}$ for $1 \mathrm{~min} 30 \mathrm{sec}$, and $72^{\circ} \mathrm{C}$ for 1 minute, with a final elongation step of $60^{\circ} \mathrm{C}$ for 30 min. Products were separated using an ABI 3100 Genetic Analyzer (Applied Biosystems) using a mix of $1 \mu 1$ diluted PCR product, $9 \mu 1$ formamide and $0.2 \mu 1$ GeneScan ${ }^{\mathrm{TM}} 500$ (-250) LIZ Size Standard (Applied Biosystems). Alleles were detected and scored using GeneMapper version 3.7 (Applied Biosystems), ensuring that only true peaks are scored by matching the peak 
morphologies to those observed during single primer tests. Allele scoring was performed automatically using the GeneMapper software and confirmed through manual inspection. Negative controls were included on each PCR plate for each multiplex to monitor for contamination, and samples with amplification problems were discarded. Forty samples were chosen randomly to test error levels; PCR, sequencing and genotyping at every locus was repeated following the same protocols.

Table 1. Microsatellite primers and multiplexes. 1(Rowe and Beebee, 2001), 2 (Garner and Tomio, 2001), 3(Berlin et al, 2000), 4 (Pidancier et al, 2002), 5 Garner unpublished.

\begin{tabular}{|c|c|c|c|c|}
\hline Multiplex & Locus & Dye & Forward 5'-3' & Reverse 5'-3' \\
\hline 1 & Rtemp $\mu 2^{1}$ & NED & ATGCTCACTATTATCTGTCCAGC & TGTTGCTTCAGGACAATTCTG \\
\hline 2 & Rtemp $\mu 1^{1}$ & VIC & TCTCTCTTCTTTGTTCCCTGAGC & CCTTGAGAGGGGCAAGTAAGGC \\
\hline \multirow{4}{*}{3} & Rtemp $\mu 7^{1}$ & FAM & TCTTTCCTGCAACCCGTGTG & CCTTGTCCCTCTGGCAAAGC \\
\hline & $\mathrm{Rt} 2 \mathrm{Ca} 9^{2}$ & VIC & TCCCTTAATTGTGCATTTTTCC & CTCTTTTCCCTTATAGGTGCAG \\
\hline & $\mathrm{RtSB}^{3}$ & PET & GAGATCCATGTGTATTTATCG & CCACTGCGATTCTGACCTGTC \\
\hline & $\mathrm{RtSB}^{3} 0^{3}$ & FAM & ACAGCTATATCCGACCAC & GGAGGACATAAGTTCAATAA \\
\hline \multirow{2}{*}{4} & Rtemp $\mu 8^{1}$ & FAM & ACCCAGTTGAATGCGGAAGC & TCTGATGGTACAGGAATGGGGG \\
\hline & $\mathrm{RtSB}_{14}^{3}$ & PET & TGTGTCCAGCAATGAATGTTA & GCAGAGTTACAGCCAAGGAA \\
\hline 5 & $\mathrm{Rt} 2 \mathrm{Ca} 2-28^{5}$ & VIC & GAGAGGGTCAACTCACAAGACC & GCGTTTACCATGTGCATGTC \\
\hline \multirow{3}{*}{6} & $\mathrm{Rt} 2 \mathrm{Ca} 30^{5}$ & VIC & AAGGAAAACAAAGTCATTAAACCA & TCCATCCAACCACAAGTAAGAA \\
\hline & ${\text { Rtemp } \mu 5^{1}}^{1}$ & FAM & GATCCACGTATGAGGATAGTTCAG & AGCAGGACTTGTGGACTGCG \\
\hline & $\mathrm{Rt} \mu \mathrm{P}^{4}$ & PET & TCATCCCTGCTACCTAATC & AAGCAATCAGGAATAAGGGG \\
\hline \multirow{2}{*}{7} & $\mathrm{Rt} 2 \mathrm{Ca} 2-22^{5}$ & FAM & CGGCTTACAAGAGGTGGAG & AGACTCCCTTACAGGCATGG \\
\hline & Rtemp $\mu 4^{1}$ & NED & TCATGCAGGCAAAGGTCAGG & AGAACCGCTGGTGTCCCAAC \\
\hline
\end{tabular}

Analysis

Whilst egg clutch sampling should ensure that in the majority of cases, each individual sampled was from a different mating, tadpole samples from nine of the Irish populations likely included full siblings. To correct for any bias in microsatellite data attributable to full-sibling relatedness, Colony v1.3 (Wang, 2004) was used to identify full-siblings within all populations. All but one member from each full-sibling group was removed from the final data set. The remaining data set with all full siblings removed consisted of 137 individuals ( 21 individuals removed) from the 16 English populations (mean = 8.563 individuals per population) and 234 individuals (182 individuals removed) from 
the 14 Irish populations (mean $=16.714$ individuals per population). Genotyping error was estimated from the number of allelic differences observed divided by the number of allelic comparisons made (Bonin et al, 2004). I tested each locus for linkage disequilibrium, and for deviations from Hardy-Weinberg equilibrium within each population, and across populations within Irish and English groups using GENEPOP v2.1 (Raymond and Rousset, 1995). Where necessary, Bonferroni corrections for multiple tests were applied (Rice, 1989).

Geographical distance matrices between pairs of populations were produced using the Geographic Distance Matrix Generator v1.0 (Ersts, 2008). Correspondence analysis was implemented in GENETIX v. 4.04 (Belkhir et al, 1996) to visualise the genetic distance (based on allelic composition and frequency) between British and Irish individuals. At this stage, a single Irish population was shown to be an outlier with respect to all other Irish populations, and so was removed from the following analyses. Measures of population variability; allelic richness, observed and expected heterozygosity, $\mathrm{F}_{\mathrm{IS}}$ and $\mathrm{F}_{\mathrm{ST}}$ were calculated and compared for each population group using FSTAT v2.9.3.2, with 100,000 permutated datasets (Goudet, 1995). Number of alleles, allelic richness and observed heterozygosity were calculated individually for each locus and each population using GENEPOP v2.1. I tested for isolation by distance by comparing pairwise $\mathrm{F}_{\mathrm{ST}}$ values with geographical distances within and between British and Irish frogs using nonparametric Mantel's tests (Mantel, 1967) (implementing Spearman's rank correlation coefficient) in GENEPOP v2.1 (Raymond and Rousset, 1995).

\section{Results}

Genotyping error was estimated to be $1.7 \%$ (Bonin et al, 2004). Irish populations where tadpoles had been sampled showed strong linkage between many loci that was not present in populations where eggs had been sampled. After full siblings were removed from the data set following analysis using Colony v1.3 (Wang, 2004), linkage disequilibrium tests were re-run and no significant linkage was found between any of the 14 loci analysed. Global tests for heterozygote deficiency showed that no loci were out 
of Hardy-Weinberg equilibrium after the application of the Bonferroni correction. There was no consistent pattern of populations out of Hardy-Weinberg equilibrium; only two English and three Irish populations were out of equilibrium after Bonferroni correction; moreover, these populations were in equilibrium at the majority of the loci.

Analysis incorporating information for all fourteen microsatellite loci indicated a significant reduction in allelic richness, expected heterozygosity and observed heterozygosity in Irish populations compared to the English populations (Table 2). These patterns held across the majority of loci (Figs. $2 \& 3$ ), and were consistent across populations (Figs. 4 \& 5). No significant difference was detected in $\mathrm{F}_{\mathrm{ST}}$ or $\mathrm{F}_{\text {IS }}$ between Irish and English populations (Table 2). No evidence for isolation by distance was identified within British or Irish populations, however significant isolation by distance was detected among British and Irish populations $\left(\mathrm{F}_{\mathrm{ST}}=0.0967+0.0002\right.$ (distance), $\mathrm{R}^{2}=0.330$, one-tailed $\mathrm{p}$ values $=0.000$ and 1.000$)$. The majority of alleles found in eastern Irish populations were a subset of those found in England; across all 14 loci (total of 195 alleles detected in this study), 14 private alleles occurred in Irish populations (total alleles detected=116) while 79 private alleles occurred in English populations (total alleles detected=181).

Table 2. Comparison of genetic variation in England and Ireland. Allelic richness, observed $\left(\mathrm{H}_{\mathrm{O}}\right)$ and expected $\left(\mathrm{H}_{\mathrm{S}}\right)$ heterozygosity, $\mathrm{F}_{\mathrm{IS}}$ and $\mathrm{F}_{\mathrm{ST}}$ are given for British and Irish populations. Probability values given relate to two-tailed tests comparing British and Irish population groups.

\begin{tabular}{|l|r|r|r|}
\hline & England & Ireland & p-values \\
\hline Allelic richness & 1.691 & 1.615 & $\mathbf{0 . 0 0 2}$ \\
\hline H $_{\mathbf{O}}$ & 0.635 & 0.556 & $\mathbf{0 . 0 0 1}$ \\
\hline $\mathbf{H}_{\mathbf{S}}$ & 0.698 & 0.619 & $\mathbf{0 . 0 0 6}$ \\
\hline $\mathbf{F}_{\text {IS }}$ & 0.090 & 0.102 & 0.656 \\
\hline F $_{\text {ST }}$ & 0.102 & 0.112 & 0.677 \\
\hline
\end{tabular}




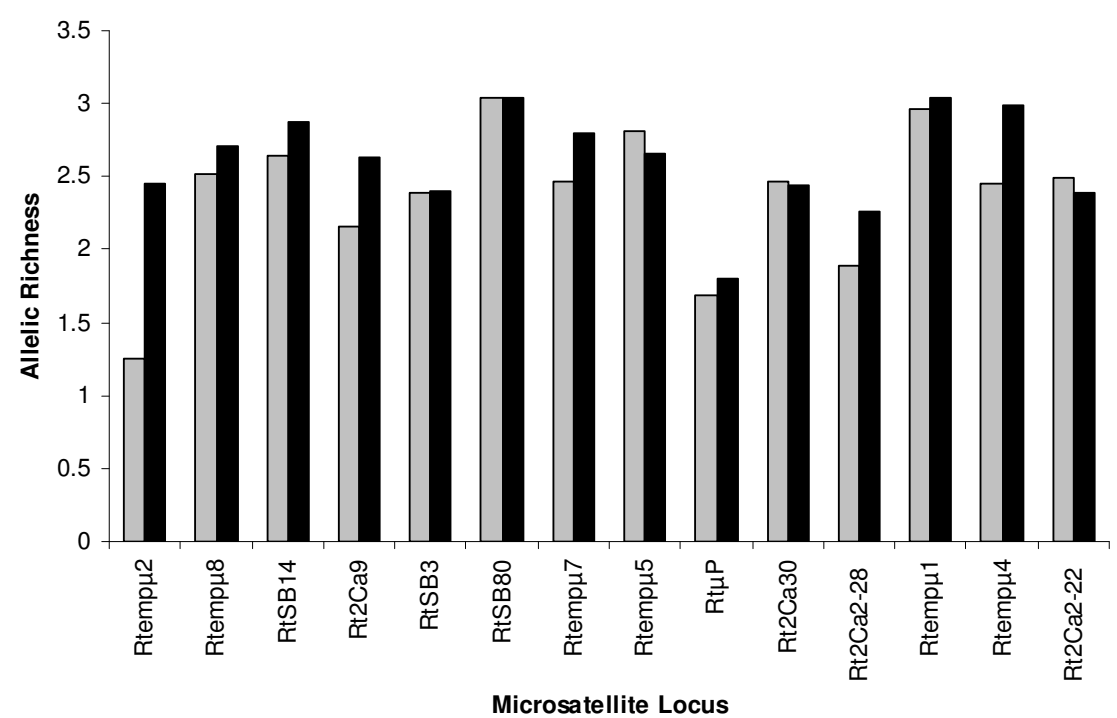

Figure 2. Allelic richness at each locus averaged across population groups. Irish populations are shown by grey columns and English populations by black columns.

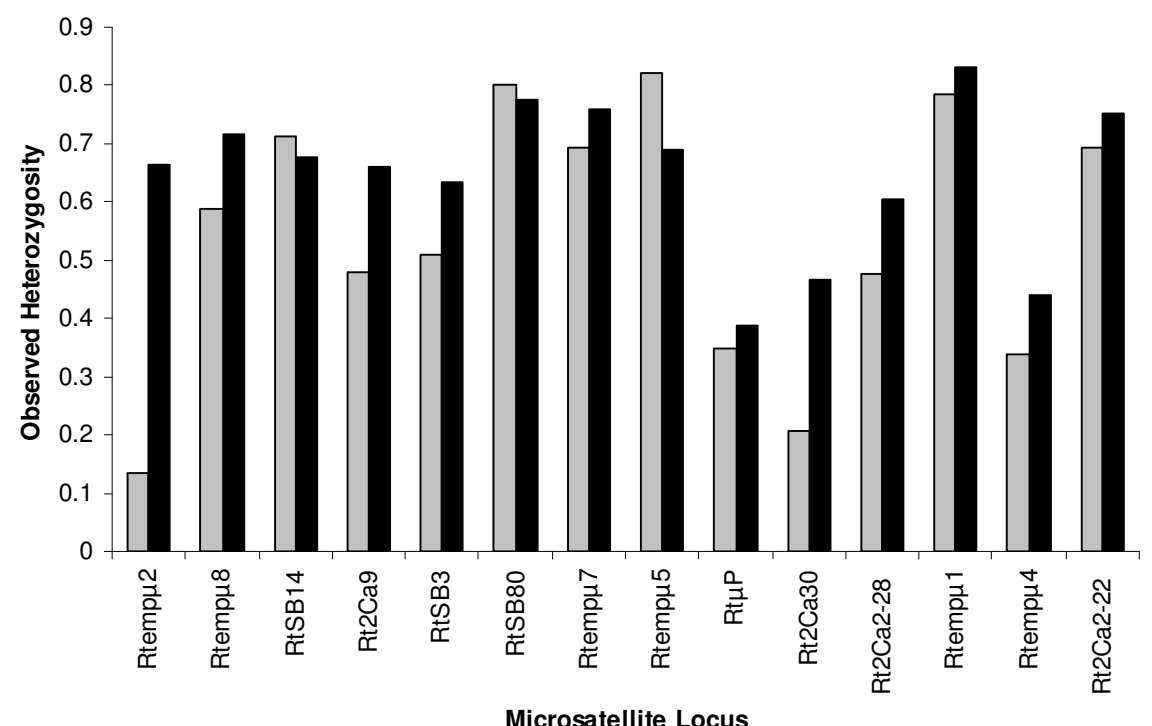

Figure 3. Observed heterozygosity at each locus averaged across population groups. Irish populations are shown by grey columns and English populations by black columns. 


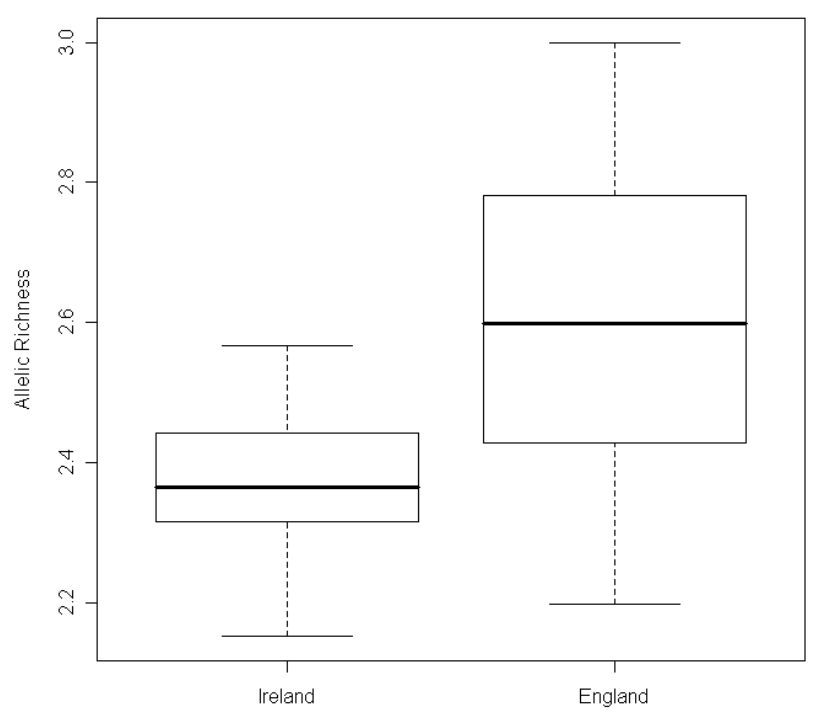

Figure 4. Box and whisker plot showing the distribution of allelic richness within population groups.

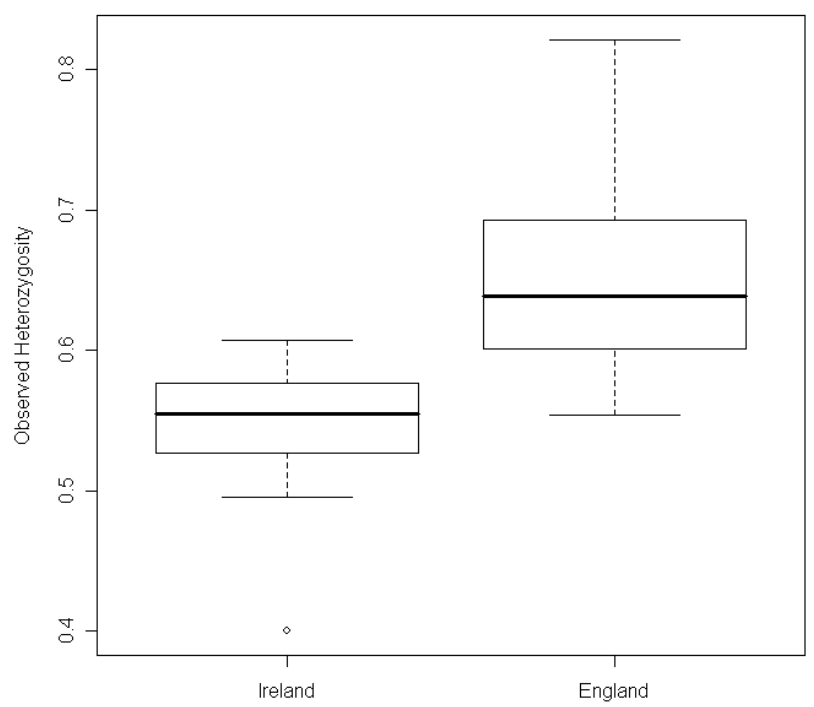

Figure 5. Box and whisker plot showing the distribution of observed heterozygosity within population groups.

Correspondence analysis performed in GENETIX grouped Irish and English individuals based on source island, and detected lower diversity within the Irish samples (Fig. 6), despite the fact that the two axes combined only account for $6 \%$ of the variation in the dataset. However, one Irish population (removed from all other analyses) was an outlier 
from the Irish group, appearing closer to the English group (Fig.6). When the owner of this pond was contacted; they stated that their pond had in fact been stocked with frog spawn that had been translocated several years previously from Worcester in England.

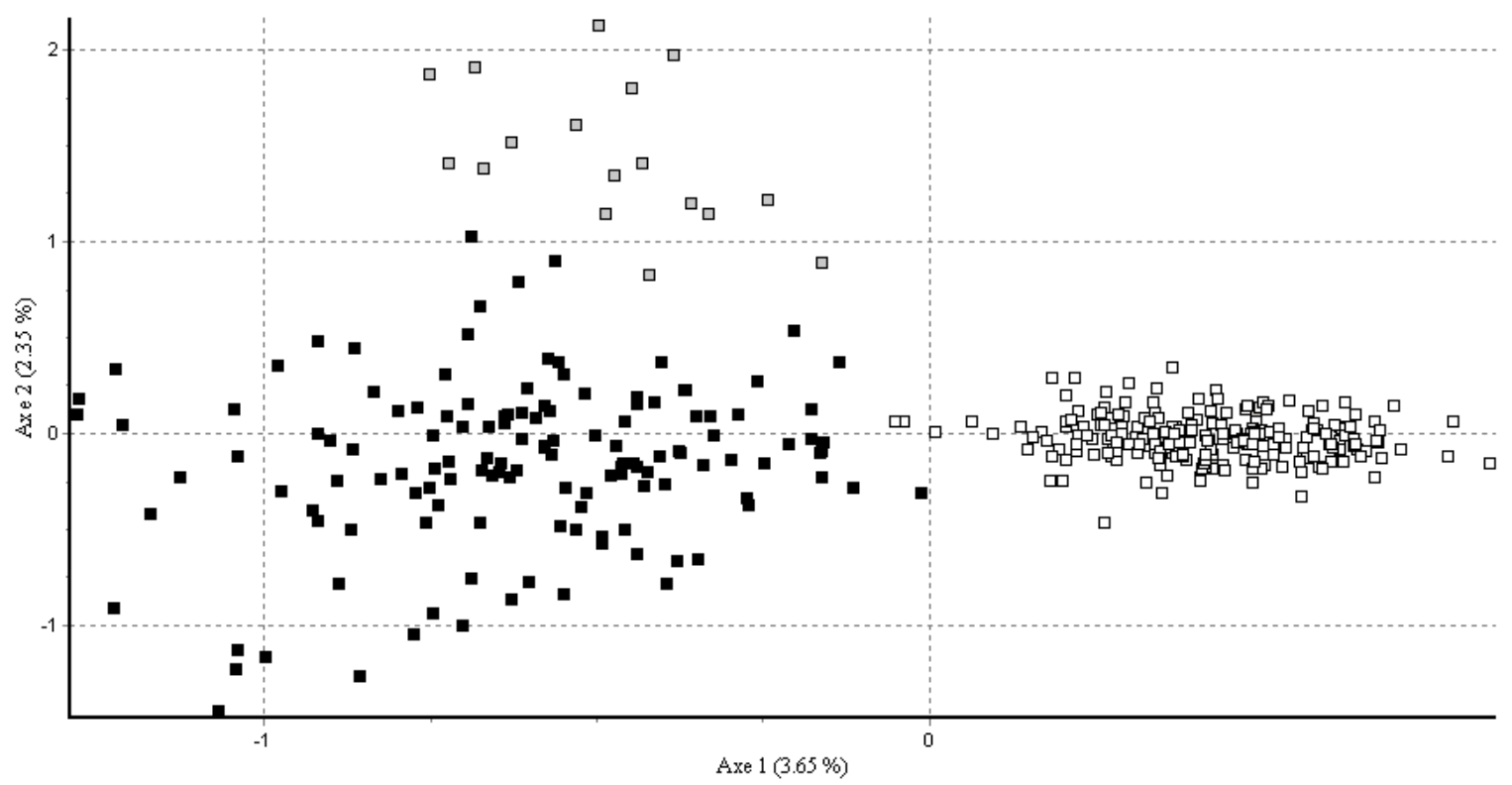

Figure 6. Correspondence analysis of English and Irish populations. Graph generated using

GENETIX to show correspondence analysis demonstrating distinct grouping of British (Black squares) and Irish (white squares) individuals, and reduced diversity within the Irish samples. Individuals from the Irish population that was translocated from Worcester in Britain are also shown (grey squares).

\section{Discussion}

Previous findings from mtDNA indicated that a dual colonization of Ireland may have occurred, with some individuals in the South West of Ireland descendant from an Irish glacial refugium, and others descendant from a Western European expansion event. Mitochondrial DNA indicated that Eastern Irish and British common frogs likely share a common ancestry, however microsatellites have revealed differences in genetic variation between the two islands that were not detectable using mtDNA. The data demonstrate a small but significant reduction in genetic diversity in Irish populations compared to English. Allelic richness, expected and observed heterozygosity are all significantly lower in Irish compared to English populations, and these findings are consistent with a severe, or recent bottleneck (Nei et al, 1975). Allelic richness is relatively sensitive to 
reductions in population size; Nei et al. (1975) show that even large founder events (100 to 1000 individuals) can lead to a loss of $40-60 \%$ of alleles. However, both theory and empirical experimentation show that a reduction in heterozygosity requires an extreme or prolonged bottleneck (Leberg, 1992; Nei et al, 1975). Ireland is a relatively isolated island within Europe, and the closest land-mass through which colonisation could have occurred is Britain. The significantly lower genetic diversity found in Ireland compared to England could be indicative of a small founder event into Ireland, and so provides some speculative support for a secondary recolonisation of Ireland via Western Europe and possibly Britain. However, further sampling throughout Europe for microsatellite analysis would be necessary to confirm the source population.

Estimates of $\mathrm{F}_{\mathrm{IS}}$ and $\mathrm{F}_{\mathrm{ST}}$ indicate similar within and between population structuring in Irish and English samples, suggesting levels of gene flow do not differ between sampling regions. Modern gene flow between England and Ireland is expected to be negligible, and a strong pattern of isolation by distance between the two locations supports this. The obvious exception to this is the Irish population that was identified as having been anthropogenically founded from an English population; this is most likely a rare event, but it conveniently served to validate the data obtained in this study. A lack of gene flow can allow divergence between populations under drift or selection (Avise, 1994), but for the most part, the alleles present in Ireland represent a subset of those in England, and many more private alleles were identified in English populations than in Irish populations. However, some private alleles $(n=14)$ were found in Ireland; it is likely that this can be explained by a combination of genetic drift, and the absence of gene flow. There was no evidence from mtDNA of individuals from the distinct Southern Irish lineages being found as far North and East as the populations sampled in this study (Dublin), thus there is no evidence of introgression between lineages in this region. Extending the microsatellite study to include the Southern Irish lineages, could provide interesting information on the level of gene flow within Ireland between refugial populations and colonizers. 
Variation in mtDNA has previously been used in conservation for the identification of Evolutionary Significant Units (ESUs), a method of assessing conservation priority based on historical population structure in order to maintain evolutionary heritage (Moritz, 1994). On this basis, English and Eastern Irish populations would form a single ESU. Another form of conservation unit, Management Units (MUs) address current population structures based on divergence of allele frequencies regardless of their evolutionary distinctiveness (Moritz, 1994). Under these criteria, English and Eastern Irish common frog populations could be categorized as separate MUs. It is evident from this study that mtDNA and microsatellites can give a different picture of population groups, and that both fast and slow evolving markers are needed to identify the distinctiveness of populations.

\section{Conclusions}

The microsatellite data are compatible with a recolonisation event in Eastern Ireland, as Eastern Irish populations have similar microsatellite alleles to British populations but lower genetic diversity, as would be expected from the founder events that typically occur during colonisation. Correspondence analysis demonstrates that Irish individuals can be clearly distinguished from British individuals on the basis of allelic frequency and the presence of some private alleles. This is expected, as Irish common frogs share similar colonisation history, and have negligible gene flow with British populations. These data demonstrate that studies of population differentiation using slow-evolving markers (such as mtDNA) can miss differences in genetic composition and diversity between populations that can be identified by faster-evolving markers (such as microsatellites). This study suggests that both types of markers are necessary for understanding phylogenetic histories at a finer scale. 


\title{
CHAPTER 4: The impact of Ranavirus infection on common frog populations in the UK
}

\begin{abstract}
Mass mortalities caused by Ranavirus have occurred in common frogs (Rana temporaria) in the UK since the 1980s. This study follows up archived records from 1996 to 2008 of common frog mortalities likely caused by Ranavirus, and demonstrates that public reports of probable Ranavirus-related frog mortalities are highly reliable. Such reports are confirmed both by observations of diagnostic skin ulceration, and by PCR screening for infection. There is preliminary evidence that common frog populations respond differently to the emergence of disease; emergence may be transient, catastrophic, or persistent despite recurrent mortalities. I evaluated whether populations with a history of recurrent disease had experienced reductions in population size, compared to those with no history of disease, using a Generalised Linear Model. This study provides evidence of localised population declines in this species, which are best explained by the presence of Ranavirus infection. Larger populations experienced larger declines, whereas habitat age had no significant effect.
\end{abstract}




\section{Introduction}

The Iridoviridae family consists of five genera; Iridovirus, Chloriridovirus, Ranavirus, Lymphocystivirus and Megalocytivirus (Murphy et al, 1995). Of these, only Ranavirus is known to affect amphibians. Unsolicited reports of unusual and often large-scale common frog (Rana temporaria) mortalities have been received by various conservation organisations in England since the 1980s. The Frog Mortality Project was initiated in 1992 at the Institute of Zoology (in association with Herpetofauna Consultants International Ltd.) and determined that these mortalities were caused by a virus belonging to the Ranavirus genus (Cunningham et al, 1996), specifically frog virus 3 (FV3), the type species for Ranavirus (Hyatt et al, 2000). The mortalities originally occurred most often in the South-East of England, however the range has since expanded to the North and West of Britain (Cunningham, 2001). FV3 isolates from Rana temporaria in the United Kingdom are similar to North American isolates, based on major capsid protein sequence data (Hyatt et al, 2000). This has led to speculation that the virus was introduced to the UK from North America, potentially due to translocations of the bullfrog (Rana catesbiana) or goldfish (Carassius auratus) for the pet trade (Cunningham et al, 2003).

Infection of $R$. temporaria with Ranavirus can cause two disease syndromes; ulcerative skin syndrome, characterised by dermal ulceration (Fig. 1) and haemorrhagic syndrome, characterised by systemic haemorrhaging within the skeletal muscles and visceral organs (Cunningham et al, 1996). Skin ulceration can become so severe that it leads to the breakdown of limbs (Fig. 1). Other signs of ulcerative skin disease include lethargy and emaciation, although these alone are not diagnostic. Mortality levels can be high; Green et al. (2002) observed over 90\% mortality in their study of 25 Ranavirus-associated amphibian (9 species) mass mortality events. However, mortalities are often restricted to a small geographical area, for example a single pond (Carey et al, 1999). Although infection can lead to mass mortalities within a population (Cunningham et al, 1996), some individuals are thought to be able to survive infection, as scarring, characteristic of 
healed skin ulcers, has been found (Fig.2). Ranavirus isolates from the UK grow in vitro between $8-30^{\circ} \mathrm{C}$, with slower replication below $15^{\circ} \mathrm{C}$ and the fastest replication at $30^{\circ} \mathrm{C}$ (Cunningham, 2001). Reflecting this temperature dependence, deaths caused by Ranavirus occur predominantly in the summer months (Chinchar, 2002; Green et al, 2002).

a)

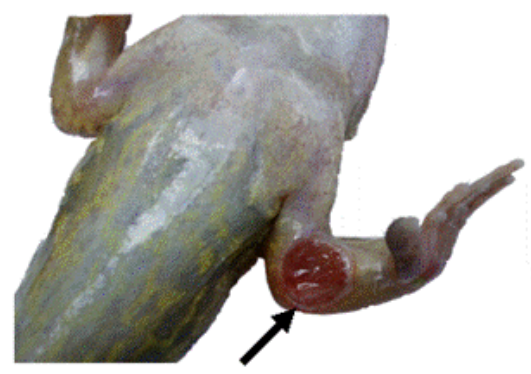

b)

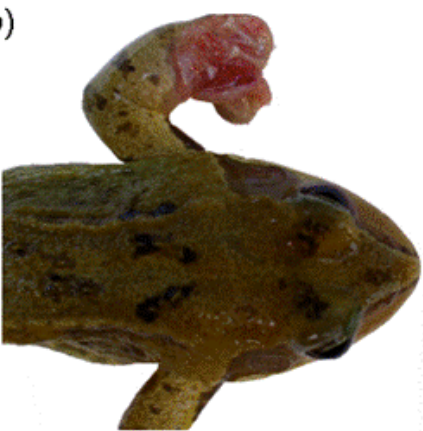

Figure 1. Lesions characteristic of ulcerative disease syndrome caused by infection with Ranavirus. (a) shows ulceration of the left forelimb (b) shows severe ulceration of the left forefoot with loss of all digits.

a)

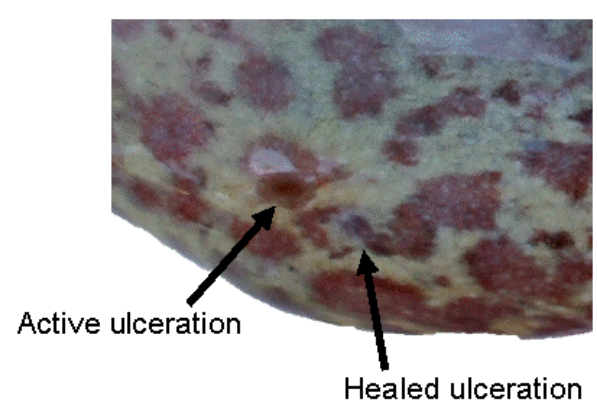

b)

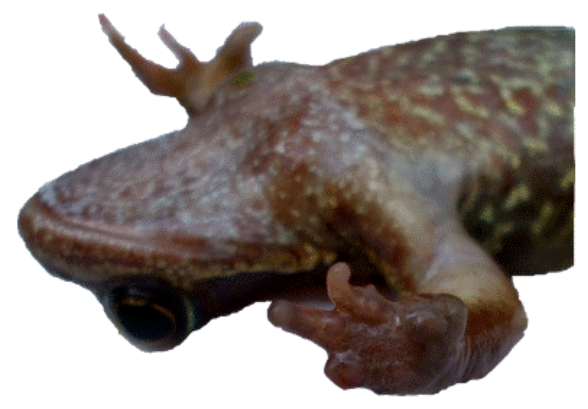

Figure 2. Scarring characteristic of healed skin ulceration. (a) shows an active ulceration next to an ulcer scar on a ventral view, (b) shows severe scarring of the right forefoot.

Mass mortalities in wild populations that are caused by Ranavirus are known to have occurred in many different amphibian species (Green et al, 2002). The best studied examples are mortalities of common frogs in the UK (Cunningham et al, 1996) and tiger salamanders (Ambystoma tigrinum) in North America and Canada (Bollinger et al, 1999; Jancovich et al, 1997). Annual recurrence of the disease has been noted in both of these species (Daszak et al, 1999). Despite causing high levels of mortality, Ranavirus has not 
yet been linked to long-term population declines (Daszak et al, 2003), and iridovirusrelated mortalities have to date not been detected in any species which is considered in decline (Green et al, 2002). Although research has shown unequivocally that Ranavirus causes mass mortalities, there remains a lack of traceable studies from which to reliably establish whether infection can cause population declines. This study aims to determine whether common frog populations in the UK have undergone population declines due to recurrent Ranavirus infection. This study takes advantage of archived records of common frog mortalities from the Frog Mortality Project, and follows up cases of probable Ranavirus infection for the period 1996 to 2008. Cases of recurrent disease are investigated further by looking for visible signs of infection of adult frogs, and by screening frog carcasses for the presence of Ranavirus using standard polymerase chain reaction (PCR) detection. The number of frogs found in these populations before and after disease emergence are compared with population size changes in uninfected ponds over the same time frame.

\section{Methods}

Identification of Ranavirus infection from archived records

Pond owners throughout the UK regularly submit records of amphibian mortalities to the charity Froglife through the Frog Mortality Project (www.froglife.org). Archives of records were searched for ponds where frogs had apparently been infected with Ranavirus, using the following criteria:

a1. Experienced one or more mass mortality of common frogs in 1997 or 1998.

a2. Mass mortality occurred in the summer months (May-September).

a3. At least one diagnostic sign of Ranavirus infection (Skin ulceration or systemic haemorrhaging) recorded for the majority of frogs involved in the mass mortality.

a4. Located in the South-East of England, within 80 miles of London.

a5. Urban or sub-urban location. 
In total, 70 such ponds were identified; the pond owners were contacted for further, upto-date information in Autumn 2005. The following criteria were then used to identify suitable study populations that had experienced recurrent disease:

b1. Frog population still present, and breeding annually, in the original pond.

b2. Independent confirmation of at least one diagnostic sign during the original mass mortality.

b3. Further deaths reported since the original mass mortality, with at least one diagnostic sign and occurring no less often than one frog every two years.

b4. Willingness to be involved with the research project for a minimum of 3 years. In total, 18 common frog populations were identified which satisfied all the criteria, these populations were termed Ranavirus-positive $(\mathrm{Rv}+)$ populations. For the purpose of this study, control populations with no history of disease were also required. An appeal was initiated for such ponds through local newspapers, Froglife and personal contacts, and sixteen Ranavirus-negative ( $\left.\mathrm{Rv}^{-}\right)$populations were identified based on the fulfilment of the following criteria:

c1. Owners had lived at the same address since at least 1997.

c2. Owners had strong awareness of their frog population.

c3. Owners had never identified any signs that may be indicative of Ranavirus infection (skin ulceration, systemic haemorrhaging, lethargy, emaciation, unusual mortalities not attributable to other causes of death).

c4. Located in the South-East of England, within 80 miles of London.

c5. Urban or sub-urban location.

c6. Common frog population breeding annually in the same pond since at least 1997.

c7. Willingness to be involved with the research project for a minimum of 3 years. Identification of Ranavirus infection from pond visits

A sub-sample of $\mathrm{Rv}+(\mathrm{n}=8)$ and $\mathrm{Rv}-(\mathrm{n}=9)$ populations were visited in between May and July 2007. Between five and ten adult frogs (over 15 grams) from each population were caught by hand and observed for evidence of Ranavirus infection; the number with active or healed skin ulceration were recorded. Those with both active and healed ulcers 
were recorded only as having active ulcers to avoid duplication of results. Skin ulceration caused by Ranavirus typically results in rounded lesions (Fig. 1), and the resulting scarring (Fig. 2) is identifiable primarily by the shape. Incidences of probable traumatic injury were also recorded, including limb breakages and amputations, skin tears, piercing injuries consistent with bird predation, and septic or swollen digits. Scars consistent with probable traumatic injury were identified as those with a non-rounded shape, such as would occur from skin tears or piercing. Numbers of frogs with active or healed skin ulceration, and numbers with traumatic injuries, were compared between $\mathrm{Rv}+$ and Rv-populations using Chi-squared contingency tests.

\section{Confirmation of Ranavirus infection by Polymerase Chain Reaction (PCR)}

Frog carcasses were obtained from ten $\mathrm{Rv}+(\mathrm{n}=16)$ and two $\mathrm{Rv}-(\mathrm{n}=3)$ populations. Cause of mortality was unknown in all cases. The liver was dissected from each carcass ensuring no contamination occurred between animals. Liver samples were used for DNA extraction using the Wizard SV96 Genomic Purification System (Promega, UK). PCR was performed twice for each sample using published FV3-specific primers which amplify a conserved region (420 base pairs) of the major capsid protein: forward: 5'GTCTCTGGAGAAGAAGAA-3'; reverse: 5'-GACTTGGCCACTTATGAC-3' (Gantress et al, 2003; Mao et al, 1996). The reaction mixture was: $2 \mu 1$ template DNA (approximately $0.3 \mu \mathrm{g} / \mu \mathrm{l}), 4 \mu \mathrm{l}$ Qiagen Master Mix (Qiagen, UK), 1.8 $\mu 1$ double-distilled and autoclaved water and $0.1 \mu \mathrm{l}$ of each primer $(100 \mathrm{pmol} / \mu \mathrm{l})$. The PCR program used was: 35 cycles of denaturation at $95^{\circ} \mathrm{C}$ for $45 \mathrm{sec}$, annealing at $52^{\circ} \mathrm{C}$ for $45 \mathrm{sec}$ and extension at $72^{\circ} \mathrm{C}$ for $45 \mathrm{sec}$ (Pearman et al., 2004). PCR controls consisted of two negative controls (no DNA) and two positive controls (known infected individuals). Total PCR products were run on a $1.2 \%$ agarose gel with $2 \mu l$ loading buffer (Microzone, UK). Samples were recorded as positive for infection with Ranavirus when both repeat PCR samples showed bands that matched the size of the positive control bands. Two carcasses from a single pond had previously been screened using transmission electron microscopy by Cunningham (2001). 


\section{The impact of disease on population size}

From the 70 pond owners who had observed a common frog mass mortality, I obtained estimated numbers of frogs killed in the initial mortality event for 53 ponds from the original archived reports. Estimated Rv+ adult population $(n=18)$ sizes were obtained from archived reports for the year prior to the initial mass mortality event (1996/7). Approximate adult population sizes in 1996/7 were also obtained from a subsample of $\mathrm{Rv}$ - population $(\mathrm{n}=10)$ pond owners. All pond owners were contacted in 2008 for a contemporary adult population size estimate. Median values were calculated due to the presence of a few outlying values for 1996/7 Rv+ population sizes. Percentage population size changes from 1996/7 to 2008 were calculated for each population. Estimates of the age of the ponds were also obtained from pond owners. To assess the variables influencing population size changes in the sampled ponds, I used a Generalised Linear Model (GLM) with normal error structure implemented in MLwiN v.2.0.01 (Rasbash et al, 2004). I used percentage population size change as the response term, which was $\log _{10}$ transformed to normalise residuals. Diseased status (Rv+, Rv-) was fitted as a categorical variable, and population size (from 1996/1997 estimates) and pond age were fitted as continuous variables. All variables were entered and dropped sequentially until only those that explained significant variation remained (resulting in a minimal model, c.f. Sokal and Rohlf, 1995). Each dropped term was then re-entered into the model to obtain p-values, and to confirm that significant terms had not been wrongly excluded. Biologically relevant two-way interactions were also tested, but did not contribute significantly to the explanatory power of the model and are not discussed further. The significance of effects was tested using the Wald statistic, evaluated against the Chi-square distribution.

\section{Results}

Of the 70 pond owners contacted, 32 had to be excluded as the original owners had moved, the pond had been filled in, or they were not willing to participate in the study. Of the remaining 38, four had no frogs left since the initial mass mortality, 16 had experienced no mortalities since, and $18(\mathrm{Rv}+$ populations $)$ had experienced at least one 
Ranavirus-consistent common frog mortality every two years since the initial outbreak.

The difference in the number of frogs with active or healed skin ulceration in Rvpopulations (0/64) and in $\mathrm{Rv}+$ populations (21/53) was highly significant $\left(\chi^{2}=30.91\right.$, $\mathrm{p}<0.00001$ ) (Table 1). The number of injuries identified as most likely caused by trauma was not significantly different between Rv- (11/64) and Rv+ (9/53) populations $\left(\chi^{2}=0.05, p=0.83\right)$ (Table 1$)$. PCR results did not identify the presence of Ranavirus in any of the three carcasses obtained from Rv- populations. Of the 18 carcasses obtained from Rv+ populations, Ranavirus was identified in $50 \%$ of the animals, and 9 out of the 11 populations (Table 2 ).

Table 1. Details of adult frogs examined for signs of Ranavirus infection.

\begin{tabular}{|c|c|c|c|c|c|c|c|}
\hline \multirow{2}{*}{$\begin{array}{l}\text { Site } \\
\text { ID }\end{array}$} & \multirow[b]{2}{*}{ Location } & \multirow{2}{*}{$\begin{array}{c}\text { Disease } \\
\text { status based } \\
\text { on survey } \\
\text { data }\end{array}$} & \multirow[b]{2}{*}{ Date visited } & \multirow{2}{*}{$\begin{array}{l}\text { No. live } \\
\text { adult } \\
\text { frogs } \\
\text { caught }\end{array}$} & \multicolumn{2}{|c|}{$\begin{array}{l}\text { No. frogs with } \\
\text { skin ulceration }\end{array}$} & \multirow{2}{*}{$\begin{array}{l}\text { No. frogs } \\
\text { with } \\
\text { traumatic } \\
\text { injury }\end{array}$} \\
\hline & & & & & Active & Healed & \\
\hline $\mathrm{C} 1$ & Hampshire & $\mathrm{Rv}-$ & $08 / 07 / 2007$ & 5 & 0 & 0 & 1 \\
\hline $\mathrm{C} 10$ & Surrey & $\mathrm{Rv}-$ & $05 / 06 / 2007$ & 10 & 0 & 0 & 2 \\
\hline $\mathrm{C} 11$ & London & $\mathrm{Rv}-$ & $29 / 05 / 2007$ & 5 & 0 & 0 & 0 \\
\hline $\mathrm{C} 13$ & London & $\mathrm{Rv}-$ & $22 / 07 / 2007$ & 5 & 0 & 0 & 0 \\
\hline $\mathrm{C} 15$ & Essex & $\mathrm{RV}_{\mathrm{N}}$ & $02 / 07 / 2007$ & 8 & 0 & 0 & 1 \\
\hline $\mathrm{C} 16$ & London & Rv- & $06 / 06 / 2007$ & 10 & 0 & 0 & 1 \\
\hline $\mathrm{C} 18$ & London & $\mathrm{RV}_{\mathrm{N}}$ & $22 / 07 / 2007$ & 5 & 0 & 0 & 1 \\
\hline $\mathrm{C} 2$ & Oxon & $\mathrm{Rv}-$ & $23 / 06 / 2007$ & 6 & 0 & 0 & 2 \\
\hline $\mathrm{C} 7$ & Kent & $\mathrm{Rv}-$ & $06 / 07 / 2007$ & 10 & 0 & 0 & 3 \\
\hline \multicolumn{4}{|c|}{ TOTAL } & 64 & $\mathbf{0}$ & $\mathbf{0}$ & 11 \\
\hline \multicolumn{4}{|c|}{ MEAN } & 7.11 & $\mathbf{0}$ & $\mathbf{0}$ & 1.22 \\
\hline E11 & Middlesex & $\mathrm{Rv}+$ & $30 / 05 / 2007$ & 7 & 1 & 1 & 1 \\
\hline E13 & Surrey & $\mathrm{Rv}+$ & $04 / 07 / 2007$ & 8 & 1 & 1 & 1 \\
\hline E2 & Surrey & $\mathrm{Rv}+$ & $17 / 07 / 2007$ & 5 & 0 & 1 & 0 \\
\hline E3 & London & $\mathrm{Rv}+$ & $16 / 07 / 2007$ & 6 & 3 & 0 & 2 \\
\hline E4 & Middlesex & $\mathrm{Rv}+$ & $15 / 06 / 2007$ & 6 & 2 & 0 & 2 \\
\hline E5 & London & $\mathrm{Rv}+$ & $14 / 06 / 2007$ & 6 & 0 & 3 & 0 \\
\hline E7 & Essex & $\mathrm{Rv}+$ & $21 / 07 / 2007$ & 8 & 6 & 0 & 1 \\
\hline E8 & Kent & $\mathrm{Rv}+$ & $28 / 06 / 2007$ & 7 & 1 & 1 & 2 \\
\hline \multicolumn{4}{|c|}{ TOTAL } & 53 & 14 & 7 & 9 \\
\hline \multicolumn{4}{|c|}{ MEAN } & 6.63 & 1.75 & 0.88 & 1.13 \\
\hline
\end{tabular}


Table 2. Results of diagnostic tests for the presence of Ranavirus in frog carcasses. *=Carcasses dissected and PCR screening performed by Amanda Duffus. ${ }^{\wedge}=$ Carcasses screened by Andrew Cunningham using transmission electron microscopy (Cunningham, 2001).

\begin{tabular}{|l|l|l|l|}
\hline \multirow{2}{*}{$\begin{array}{c}\text { Population } \\
\text { ID }\end{array}$} & \multirow{2}{*}{ Disease status inferred from symptoms } & \multicolumn{2}{c|}{ Number of carcasses } \\
\cline { 3 - 4 } & & Ranavirus present & Ranavirus absent \\
\hline $\mathrm{c} 08$ & $\mathrm{Rv}-$ & 0 & $2^{*}$ \\
\hline $\mathrm{c} 16$ & $\mathrm{Rv}-$ & 0 & $1^{*}$ \\
\hline \multicolumn{2}{|c|}{ TOTAL from Rv- populations } & $\mathbf{0}$ & $\mathbf{3}$ \\
\hline $\mathrm{e} 01$ & $\mathrm{Rv}+$ & 1 & 1 \\
\hline $\mathrm{e} 02$ & $\mathrm{Rv}+$ & 1 & 0 \\
\hline $\mathrm{e} 04$ & $\mathrm{Rv}+$ & $1^{*}$ & $3^{*}$ \\
\hline $\mathrm{e} 05$ & $\mathrm{Rv}+$ & 0 & $1^{*}$ \\
\hline $\mathrm{e} 07$ & $\mathrm{Rv}+$ & 0 & $2^{*}$ \\
\hline $\mathrm{e} 08$ & $\mathrm{Rv}+$ & $1^{*}$ & 0 \\
\hline $\mathrm{e} 10$ & $\mathrm{Rv}+$ & 1 & 0 \\
\hline $\mathrm{e} 11$ & $\mathrm{Rv}+$ & 1 & 0 \\
\hline $\mathrm{e} 12$ & $\mathrm{Rv}+$ & $1^{\wedge}$ & $1^{\wedge}$ \\
\hline $\mathrm{e} 15$ & $\mathrm{Rv}+$ & 1 & 0 \\
\hline $\mathrm{e} 19$ & $\mathrm{Rv}+$ & 1 & 1 \\
\hline TOTAL from & $\mathrm{Rv}+$ populations & $\mathbf{9}$ & $\mathbf{9}$ \\
\hline
\end{tabular}

The median number of frogs killed in a mass mortality was $30.0(\min =6, \max =200$, based on 53 ponds, see fig. 3). Median population sizes in 1996/7 were: Rv+ =31, Rv$=25$; and median population sizes in 2008 were: $\mathrm{Rv}+=6, \mathrm{Rv}-=24$. The median percentage population size change from $1996 / 7$ to 2008 in the $18 \mathrm{Rv}+$ ponds was $-82.82 \%(\min =-94 \%, \max =-64.52 \%)$, compared to no change $($ median $=0, \min =$ $-53.33 \%, \max =+400 \%$ ) in 10 of the Rv-ponds (Fig. 4). The variable most strongly associated with population size change was disease status (GLM: $\chi^{2}=38.32, \mathrm{df}=1$, $\mathrm{p}<0.0001)$. Population size $(1996 / 7)$ was significantly correlated with population size change $\left(\chi^{2}=15.79, \mathrm{df}=1, \mathrm{p}<0.0001\right)$, indicating that larger populations experienced larger declines. Pond age did not explain a significant change in variation $\left(\chi^{2}=0.05, \mathrm{df}=1\right.$, $\mathrm{p}=0.83)$. 


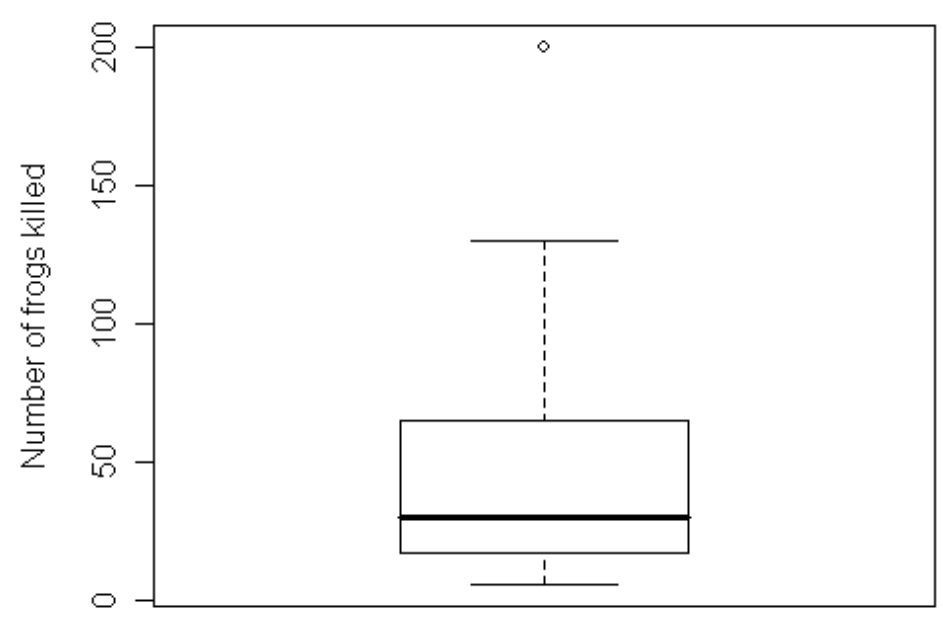

Figure 3. Numbers of frogs killed in Ranavirus mass mortality events. Box and whisker plot showing the distribution of the numbers of common frogs killed during the initial mass mortality event at 53 populations.

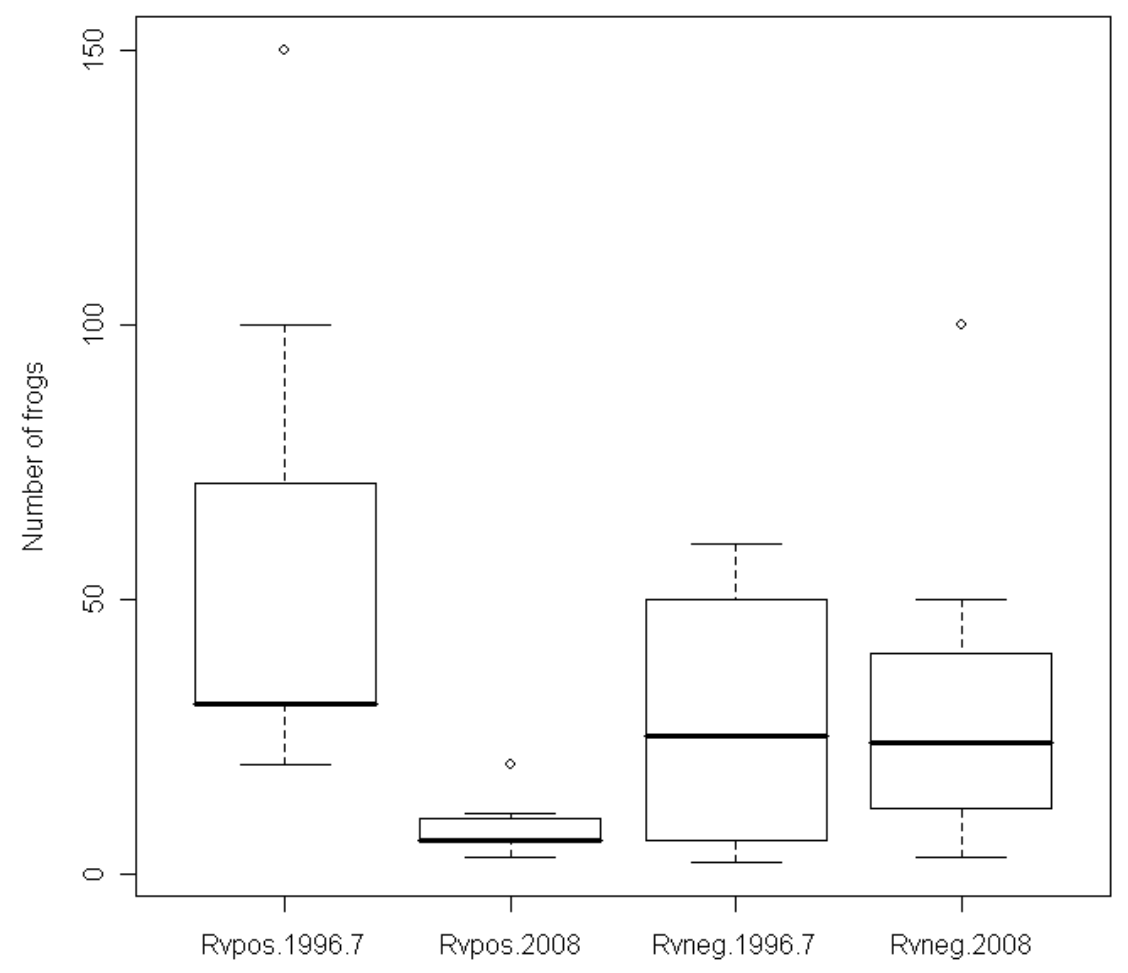

Figure 4. Population sizes from 1996 to 2008. Box and whisker plot showing public estimates of numbers of adult common frogs present in $\mathrm{Rv}+(\mathrm{n}=18)$ and $\mathrm{Rv}-(\mathrm{n}=10)$ populations in 1996/7 and 2008. 


\section{Discussion}

Variability in the impact of infection on populations

The findings from archival reports imply that the population-level response to Ranavirus emergence can vary. Some common frog populations appear to have been eradicated since the disease outbreak, some persist despite experiencing recurrent disease most years, and some populations completely recovered with no subsequent mortalities, mirroring previous findings by Cunningham (2001). The standard Susceptible/Infectious (SI) model of disease indicates that if the disease-induced death rate is greater than the reproductive rate of the host population, then the disease can regulate the host population at a stable equilibrium (Anderson and May, 1979a; Anderson and May, 1979b). This effect has been shown previously in wildebeest populations after the introduction of rinderpest to Africa (Begon et al, 1996), and in the European spruce sawfly (Gilpinia hercyniae), which is regulated at a low density in UK forests by viral infection (Baculoviridae) (Entwistle et al, 1983). If there is both frequency-dependent (e.g. sexual contact), and density dependent (e.g. social contact) transmission, then it is possible for the three outcomes above to occur (localised extinction, persistence, or recovery) (Anderson and May, 1979a; Anderson and May, 1979b). However, other factors could also influence the outcome, including variation in pathogen virulence and host resistance.

It is possible that strains of Ranavirus induce different death-rates due to dissimilar transmission rates or pathogenicity. Although different genetic lineages have been identified (Cunningham, 2001; Hyatt et al, 2000), no information is as yet available on relative virulence. The immune response to Ranavirus is both innate and acquired, and differences in the immune response may be responsible for differences in the impact of infection. For example, antimicrobial peptides have been shown to directly inhibit viral replication in Rana pipiens (Chinchar et al, 2001), though this study has not yet been replicated in other species. There is also evidence that the Xenopus Major Histocompatability Complex (MHC) class I locus (which is directly linked to the 
acquired immune response) can influence susceptibility to Ranavirus; Gantress et al. (2003) showed that young larvae are more susceptible to infection because at this age they do not express products derived from this locus. Extensive mortalities linked to Ranavirus in cultured frogs (Rana grylio and Rana tigrina) in Thailand and China led to the speculation that stressors such as high population densities and immunosuppression cause increased susceptibility in captivity (Carey et al, 1999; Kanchanakhan, 1998; Zhang et al, 2001).

However, using the data collected, it is not possible to unequivocally confirm that the populations that appear to have undergone localised extinction did so because of Ranavirus, or that the populations that recovered were indeed infected with Ranavirus. Data on extinction rates in the absence of Ranavirus infection would be useful for clarifying these results. Furthermore, a better understanding of both the host (immune capabilities) and pathogen (whether different strains in the UK have dissimilar virulence/transmission capabilities) is imperative for understanding this system. A focussed study on the correlates of disease outcome could also yield valuable results in the future.

\section{Detectability of Ranavirus in infected populations}

All populations with recurrent disease $(\mathrm{Rv}+)$ were found to have at least one frog with active or healed skin ulceration, indicative of Ranavirus infection, whilst no such lesions were identified in frogs from Rv- populations. Levels of traumatic injury were not significantly different between $\mathrm{Rv}$ - and $\mathrm{Rv}+$ populations, indicating that traumatic injuries were not incorrectly identified as Ranavirus lesions in the $\mathrm{Rv}+$ populations. Consistency between archival reports from pond owners and observations during visits indicates that public identification of the signs of Ranavirus infection is reliable. Despite owners of $\mathrm{Rv}+$ populations reporting one or more common frog death at least every two years, few carcasses were obtained for PCR screening due to the logistics of collecting such samples prior to degradation. However, infection with Ranavirus was confirmed in frogs from nine $\mathrm{Rv}+$ populations. This finding confirms the presence of the suspected 
disease agent, and provides supporting evidence for diagnosis based on the selected criteria. Half of the carcasses obtained from $\mathrm{Rv}+$ populations were negative for the presence of Ranavirus using PCR detection. It is possible that the prevalence of the disease within these populations is close to $50 \%$, or that the screening method is not sufficient to detect low-levels of infection.

\section{Population size change}

The numbers of frogs killed by the initial mass mortality events were highly consistent with equivalent data published previously. Cunningham (2001) found that within ten UK populations (one of which is also in this dataset), the median number of common frogs killed in an initial mass mortality event in 2001 was $34(\min =8, \max =156)$. The GLM indicates that disease status is a strong predictor of population size change. Within populations which experienced recurrent disease, the common frog population size was dramatically reduced 10-11 years after the first disease outbreak. Results from Rvpopulations indicate that common frog population numbers are stable in this geographical region where Ranavirus disease is not detected. There remains a lack of evidence of widespread long-term population declines caused by Ranavirus, however the data from this study indicates that the virus can cause localised population declines in Rana temporaria. Many studies show that larger populations tend to harbour greater genetic variation, which has been linked to stability under environmental change (e.g. Frankham et al, 2002). However, my analysis indicates that larger populations are more likely to show larger population declines, so individuals are equally as susceptible regardless of population size. Studies have also shown that older habitats may provide better environments, in particular due to higher food availability or lower disturbance levels. For example, in a study by Barone and Frank (2003), the nutritional condition of beetles (Poecilus cupreus) was found to be best explained by habitat age, which accounted for over $70 \%$ of the variation; the authors explained this result by the beneficial effects of greater vegetation cover in providing higher humidity. However, I found no evidence of such an effect on the impact of Ranavirus infection. 


\section{Conclusions}

Consistency between archived reports from pond owners, observations of skin ulceration, and PCR screening for infection indicates that the disease statuses of the study populations were correctly identified. Common frog populations may respond differently after the emergence of Ranavirus; some populations appear to recover and not experience future mortalities, some populations experience recurrent mortalities, and some populations may be eliminated. There is good evidence that localised population declines have occurred in populations experiencing recurrent mortalities, and that these declines are best explained by disease status. There is no evidence that increased habitat age and population size result in more stable populations under infection. 


\title{
CHAPTER 5: Neutral genetic variation in wild populations of common frogs (Rana temporaria) which have been subject to disease outbreaks (Ranavirus).
}

Submitted (December 2008) for publication as:

Teacher, A.G.F., Garner, T.W.J., Nichols, R.A. Unexpected changes to the population genetic structure of wild common frogs (Rana temporaria) following disease outbreaks (Ranavirus). Molecular Ecology

\begin{abstract}
For this section of the study, I used 14 microsatellite loci to investigate the impact of a viral disease (Ranavirus) on the population genetics of wild common frogs (Rana temporaria). Populations with a history of Ranavirus mortalities were compared with populations with no history of infection. I found surprising results: infected ponds showed significantly elevated $\mathrm{F}_{\mathrm{IS}}$ (homozygote excess), significantly reduced relatedness, and no detectable effect on allelic richness. I hypothesise that the elevated $\mathrm{F}_{\mathrm{IS}}$ and reduced relatedness are consequences of assortative mating between uninfected individuals, and that allelic richness is maintained by immigration from nearby populations. Simulations indicate that the elevated $\mathrm{F}_{\mathrm{IS}}$ cannot be explained by population size reductions, but can indeed be explained by assortative mating (even if a mate choice locus is unlinked to the genetic markers). These results indicate that the impacts of emerging infectious diseases may extend more widely than the reduction of population size.
\end{abstract}




\section{Introduction}

Amphibian populations are showing an ongoing decline worldwide, caused in part by emerging infectious diseases (Houlahan et al, 2000; IUCN, 2004; Stuart et al, 2004). Diseases can affect populations rapidly, causing sudden population crashes and even extinctions (IUCN, 2004). Such population bottlenecks may result in a loss of diversity (Frankham et al, 2002). Theory predicts a marked decrease in allelic richness (number of alleles) and a more limited decrease in diversity (expected heterozygosity), as rare alleles are the most frequently lost and contribute less to average heterozygosity (Nei et $a l, 1975)$. This pattern has been identified in manipulated laboratory populations of Drosophila (England et al, 2003), as well as in natural populations, for example in prairie chickens (Tympanuchus cupido) following habitat loss (Bellinger et al, 2003). However, studies of natural populations which have undergone bottlenecks often appear to be at odds with these theoretical expectations. A compelling example is Queney et al's (2000) allozyme and microsatellite study on wild European rabbits (Oryctolagus cuniculus). No loss of genetic diversity was identified in populations which had undergone a bottleneck of approximately $90 \%$ due to rabbit viral haemorrhaghic disease, perhaps because the population size remained high enough to retain diversity (Queney et $a l, 2000)$. The interaction between disease, mating systems, and genetic diversity is particularly interesting; many studies indicate that an individual that is inbred (as measured by pedigree) or has a high level of multilocus homozygosity is more susceptible to disease (e.g. sea lions (Zalophus californianus), Acevedo-Whitehouse et al, 2003; and Soay sheep (Ovis aries), Coltman et al, 1999). The same effect can be observed at the level of the population. For example, Pearman and Garner (2005) present evidence that Italian agile frog (Rana latastei) populations with reduced genetic diversity experience higher mortality rates when exposed to Ranavirus. Thus disease has the potential to cause bottlenecks that reduce the population size to such that a degree of inbreeding is inevitable, and inbreeding can, in turn, lead to an increase in susceptibility to disease. Inbreeding depression (a reduction in fitness caused by inbreeding, see Charlesworth and Charlesworth, 1987) has not to my knowledge been identified in 
common frogs (Rana temporaria), and this species is thought to be primarily random mating (Elmberg, 1987; Elmberg, 1991). However, one study has found that artificially outbreeding common frogs from different populations resulted in smaller and more malformed progeny, suggesting outbreeding depression (Sagvik et al, 2005).

This study investigates the impact of disease outbreaks on genetic variation in wild populations of common frogs (Rana temporaria). Ranavirus (family Iridoviridae) is known to cause mass mortality events in many amphibian species, including the common frog, though there are no published records of the long term population impacts (Cunningham et al, 1996; Green et al, 2002). The first outbreaks of Ranavirus in England were recorded in the early 1980s, and were accompanied by unusual and often large scale mortalities (Cunningham et al, 1996). The virus causes skin ulceration and/or systemic haemorrhaging and often death (Cunningham et al, 1996); however, it seems likely that some individuals can survive infection, as it is possible to find frogs with scarring consistent with previous skin ulceration. Ranavirus is known to replicate better at warmer temperatures, and deaths from Ranavirus usually occur in the summer months (Cunningham, 2001; Green et al, 2002). In this study I compare microsatellite variation in common frog populations which have experienced mortalities caused by Ranavirus $(\mathrm{Rv}+)$ with those that have not $(\mathrm{Rv}-)$. Simulations are run to show the effects on genetic diversity of a population bottleneck, and of assortative mating versus random mating, and I compare the empirical findings to those of the simulations.

\section{Methods}

\section{Sampling}

Reports of amphibian mortalities, including symptoms and months of death, have been collected by the Frog Mortality Project since 1992 (Cunningham et al, 1996). From these records, I selected ponds which had their first mass mortality in 1997 and which had experienced one or more individual mortalities most years (and at least every second year) up to 2006 when the sampling began. In order to confirm that mortalities were associated with Ranavirus, I selected ponds where the owners had reported at least one 
diagnostic symptom (skin ulceration or systemic haemorrhages) in the majority of their dead frogs (Cunningham et al, 1996). Other non-diagnostic symptoms of Ranavirus infection include lethargy and emaciation. To qualify as a disease positive population the mortalities also had to have occurred between May and September of each year. Ponds which conformed to both the symptoms and month of mortality were identified as disease positive (Rv+). Mortalities caused by Ranavirus are easily identifiable and tend to occur on a large scale. I also identified disease negative ( $\mathrm{Rv}-)$ populations by finding pond owners who had witnessed their frog populations from at least 1997 to 2006 and never seen any unusual mortalities. (The Rv- samples used for this study were the same samples as used in chapter 3, they were previously referred to as the 'English' samples).

In urban environments in England, common frog populations are frequently found in relatively isolated garden ponds. The distance between ponds included in the study ranged from 0.8 to $183 \mathrm{~km}$. Given the low migration rate over such distances (Baker and Halliday, 1999), I consider each pond to be a distinct subpopulation. I sampled $18 \mathrm{Rv}+$ and $16 \mathrm{Rv}$ - populations during the breeding seasons (February to April) of 2006 and 2007 (Fig. 1). Spawning can last about 20 days, however the majority of clutches are produced within a few days (Haapanen, 1982). I sampled one egg from each clutch present at each pond during the breeding season. Each pond was visited once within a few days of the start of breeding. Embryos were allowed to develop to Gosner stage 19 to 20 (Gosner, 1960) in order to increase DNA yield, dissected from the egg mass and the tails used for DNA extraction with the Wizard SV96 Genomic Purification System (Promega, UK). 


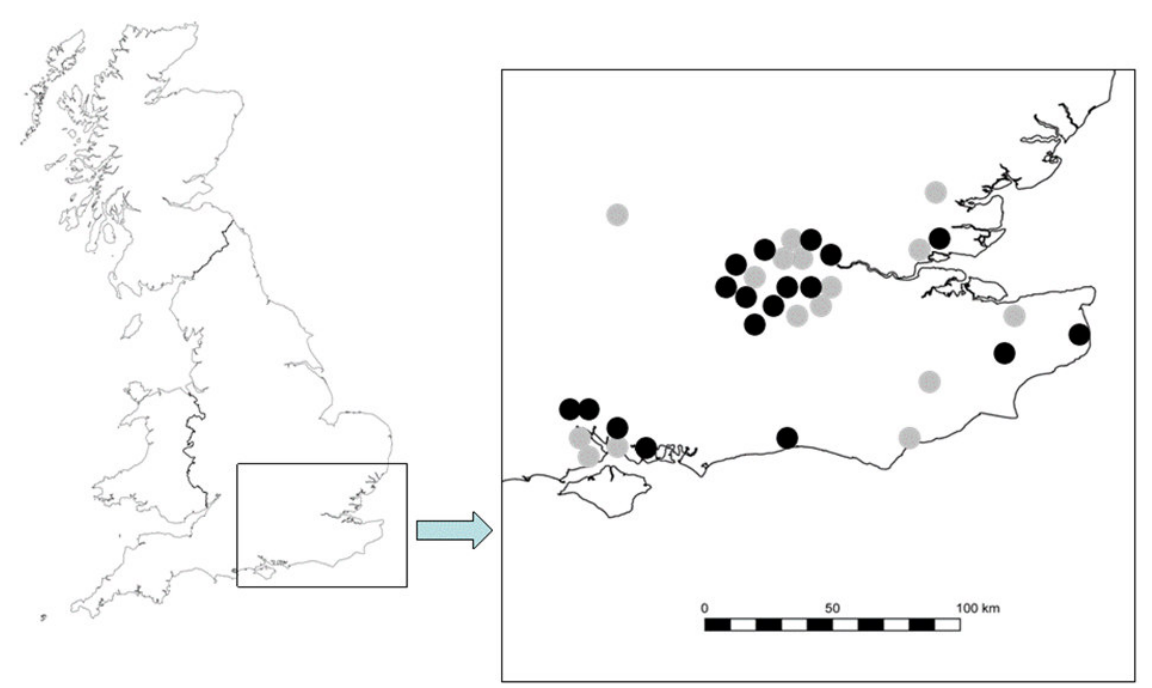

Figure 1. Map of sampling sites in England. Infected $(\mathrm{Rv}+)$ populations are shown by black circles and uninfected (Rv-) populations are shown by grey circles.

\section{Microsatellite genotyping}

14 microsatellite primer pairs were used (see Table 1 in Chapter 3 for primers and multiplex arrangements). Polymerase chain reaction (PCR) was performed using $4 \mu 1$ Qiagen Multiplex Mix (Qiagen, UK), $2 \mu$ l template DNA, $2 \mu \mathrm{M}$ of each primer, made up to $8 \mu \mathrm{l}$ with double distilled and autoclaved water. The samples were denatured at $95^{\circ} \mathrm{C}$ for 15 minutes followed by 30 cycles of $94^{\circ} \mathrm{C}$ for $30 \mathrm{sec}, 57^{\circ} \mathrm{C}$ for $1 \mathrm{~min} 30 \mathrm{sec}$ and $72^{\circ} \mathrm{C}$ for 1 minute each, followed by a final elongation step of $60^{\circ} \mathrm{C}$ for $30 \mathrm{~min}$. Products were separated using the Applied Biosystems 3100 Genetic Analyzer using $1 \mu$ diluted PCR product, $9 \mu 1$ formamide and 0.2 2 l LIZ 500 (-250) size standard (Applied Biosystems). Alleles were visualised and automatically scored using GeneMapper version 3.7 (Applied Biosystems), ensuring that only true peaks are scored by matching the morphologies to those observed during single primer tests. Automatic genotyping was verified manually to check for any discrepancies. Negative controls were performed on each PCR plate for each multiplex to monitor for contamination, and samples with amplification problems were discarded. Forty samples were chosen randomly to test error levels; PCR, sequencing and genotyping at every locus were repeated following the same protocols. 
Whilst sampling from separate egg clutches should ensure that each individual sampled is from a different mating, separate clutches were not always clearly identifiable. To alleviate potential problems that might be caused by this, I ran an analysis using Colony v1.3 (Wang, 2004) to identify full sibling groups within populations. One member of each full sibling group was left in the data set. The remaining data set with all full siblings removed consisted of 167 individuals from the $18 \mathrm{Rv}+$ populations (mean = 9.278 individuals per population) and 137 individuals from the $16 \mathrm{Rv}$ - populations $($ mean $=8.563$ individuals per population $)$.

Analysis

The genotyping error rate was estimated based on the number of allelic differences observed divided by the total number of allelic comparisons (Bonin et al, 2004). I tested for linkage disequilibrium and Hardy Weinberg equilibrium in each population across all loci and at each locus individually using GENEPOP v2.1 (Raymond and Rousset, 1995). I used FDist2 (Beaumont and Nichols, 1996) to assess whether any loci were affected by selection. The program plots expected heterozygosity against $\mathrm{F}_{\mathrm{ST}}$ and identifies any loci that fall outside the $95 \%$ confidence intervals. It was applied to the combined dataset (Rv+ and Rv-). I estimated allelic richness, observed and expected heterozygosity (Nei, 1987), $F_{S T}$ and $F_{I S}$ (Weir and Cockerham, 1984), relatedness (using an estimator strictly equivalent to Queller and Goodnight, 1989) and inbreeding corrected relatedness (Pamilo, 1984; Pamilo, 1985) and tested for significant differences between groups of Rv+ and Rv- populations using FSTAT v2.9.3.2 and 100,000 permutated datasets (Goudet, 1995). Allelic richness calculations in FSTAT enable the direct comparison of populations irrespective of sample size. These tests were repeated using a reduced dataset with loci removed that were identified by FDist2 as showing non neutral characteristics, and again after removing loci that were identified as being out of Hardy Weinberg equilibrium. Observed heterozygosity and $F_{I S}$ were calculated at each locus for each disease category, and for each population across all loci, using GENEPOP v2.1 (Raymond and Rousset, 1995). Allelic richness was estimated at each locus for each 
disease category, and for each population across all loci, using FSTAT v.2.9.3.2

(Goudet, 1995).

Simulated populations

A simulation was produced (by Prof Richard A. Nichols) using R v.2.4.0 to calculate expected heterozygosity and Weir and Cockerham's (1984) estimator of FIS. The source code is included in appendix 3. The program modelled ten overlapping generations: breeding adults could survive from one generation to the next, with a probability set by one of the parameters (1/3 in the runs presented here). The simulation modelled the real data, having 14 loci, with a maximum of 20 alleles per locus. We varied the effective population size $(\mathrm{Ne})$ to correspond to the estimated number of egg clutches in a pond before a disease outbreak (15) and after a severe decline (2). The model included a locus which could induce assortative mating. The simulation was initiated with a dominant allele, $A$, at a frequency of 0.3 , so that approximately half of the population were of each assortment phenotype ( $A A$ vs. $A a$ or $a a$ ). This locus was unlinked to any of the marker loci. A parameter, $P$, set the strength of assortment; when $P$ was set to 0.5 mating was at random. Otherwise the relative probability of an individual being selected as a mate was $P$ if they had the same assortment phenotype, and 1-P if they had different assortment phenotypes. For each parameter combination, 1000 iterations were performed. T-tests (two sample, unequal variance) were applied to test whether $\mathrm{F}_{\mathrm{IS}}$ values were significantly different at different effective population sizes, and under assortative or random mating.

\section{Results}

I calculated a genotyping error rate of $1.8 \%$; I expect these errors to have been caused by occasional allelic dropouts and human error. When working at the population level, this error rate should have little impact on analyses (Bonin et al, 2004). No consistent linkage disequilibrium was identified between loci. Two loci were out of Hardy Weinberg equilibrium after applying the Bonferroni correction (Rt2Ca30, $\mathrm{p}=0.0016$ and Rt2Ca2-22, p=0.0064); removal of these loci did not affect the results. Three loci (Rt $\mu \mathrm{P}$, Rtemp 8 and Rt2Ca30) showed unusually high or low levels (outside the 95\% 
confidence intervals) of population subdivision $\left(\mathrm{F}_{\mathrm{ST}}\right)$ when compared to heterozygosity, indicating that they may be affected by selection and therefore non neutral; the removal of these loci also did not affect the results.

There was no significant difference in allelic richness, expected heterozygosity or $\mathrm{F}_{\mathrm{ST}}$ between $\mathrm{Rv}+$ and $\mathrm{Rv}$ - populations. The estimate of $\mathrm{F}_{\mathrm{IS}}$ (which measures the excess of homozygotes over Hardy Weinberg expectations) was significantly higher in Rv+ populations compared to $\mathrm{Rv}$ - populations $(\mathrm{p}=0.009)$. Relatedness was not significantly different between $\mathrm{Rv}+$ and $\mathrm{Rv}$ - groups, however inbreeding corrected relatedness was significantly lower in $\mathrm{Rv}+$ populations $(\mathrm{p}=0.008)$. Observed heterozygosity was lower in infected populations, though the significance was borderline $(\mathrm{p}=0.06)$. These patterns were consistent when the analyses were repeated without the loci (a) identified by Fdist2 as potentially non neutral and (b) identified as being out of Hardy Weinberg equilibrium (Table 1). Allelic richness, observed heterozygosity and $\mathrm{F}_{\text {IS }}$ results were calculated at each locus and each population individually; the patterns described were consistent across most loci, and do not appear to have been biased by individual populations.

Table 1. Comparison of genetic variation in infected and uninfected populations. Allelic richness, observed $\left(\mathrm{H}_{\mathrm{O}}\right)$ and expected $\left(\mathrm{H}_{\mathrm{S}}\right)$ heterozygosity, $\mathrm{F}_{\mathrm{IS}}, \mathrm{F}_{\mathrm{ST}}$, relatedness and inbreeding-corrected relatedness (relc) are given for $\mathrm{Rv}+$ and $\mathrm{Rv}$ - populations. Locus selection (a) All loci $\mathrm{n}=14$, (b) Three potentially nonneutral loci identified by fdist 2 removed $n=11$, (c) Two loci out of Hardy-Weinberg equilibrium removed $\mathrm{n}=12$. Probability values given relate to two-tailed tests comparing disease positive populations with disease negative populations.

\begin{tabular}{|l|l|l|l|l|l|l|l|l|l|}
\hline & \multicolumn{4}{|l|}{ Rv- } & \multicolumn{1}{l|}{ Rv+ } & \multicolumn{3}{l|}{ p-values } \\
\hline Locus selection & $\mathbf{a}$ & $\mathbf{b}$ & $\mathbf{c}$ & $\mathbf{a}$ & $\mathbf{b}$ & $\mathbf{c}$ & $\mathbf{a}$ & $\mathbf{b}$ & c \\
\hline Allelic richness & 1.691 & 1.712 & 1.698 & 1.689 & 1.719 & 1.698 & 0.959 & 0.744 & 0.970 \\
\hline $\mathbf{H}_{\mathbf{O}}$ & 0.635 & 0.676 & 0.672 & 0.598 & 0.624 & 0.626 & $\mathbf{0 . 0 6 0}$ & $\mathbf{0 . 0 3 2}$ & $\mathbf{0 . 0 2 5}$ \\
\hline $\mathbf{H}_{\mathbf{S}}$ & 0.698 & 0.725 & 0.706 & 0.701 & 0.725 & 0.706 & 0.898 & 0.979 & 0.970 \\
\hline F $_{\text {IS }}$ & 0.090 & 0.068 & 0.049 & 0.148 & 0.140 & 0.113 & $\mathbf{0 . 0 0 9}$ & $\mathbf{0 . 0 1 0}$ & $\mathbf{0 . 0 2 1}$ \\
\hline F $_{\text {ST }}$ & 0.102 & 0.100 & 0.100 & 0.095 & 0.097 & 0.092 & 0.783 & 0.891 & 0.721 \\
\hline Relatedness & 0.172 & 0.172 & 0.174 & 0.155 & 0.159 & 0.153 & 0.626 & 0.711 & 0.558 \\
\hline Relc & -0.198 & -0.146 & -0.103 & -0.347 & -0.325 & -0.255 & $\mathbf{0 . 0 0 8}$ & $\mathbf{0 . 0 1 0}$ & $\mathbf{0 . 0 1 4}$ \\
\hline
\end{tabular}


Simulations with overlapping generations and random mating (Table 2) demonstrated the well known result that a severe reduction in population size reduces expected

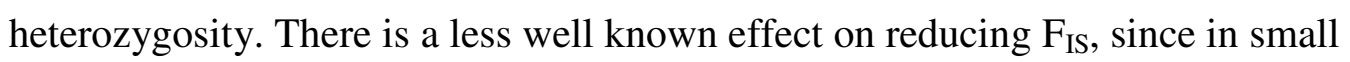
populations the fact that individuals cannot mate with themselves produces fewer homozygotes than predicted by the Hardy Weinberg proportions. Introducing assortative mating (governed by an unlinked locus) results in a small (under $3 \%$ change) reduction in heterozygosity which may often be undetectable in real populations. However, $\mathrm{F}_{\mathrm{IS}}$ at the marker loci was substantially elevated by assortative mating. A statistically significant effect is seen in large populations $(\mathrm{p}<0.0001)$; the value of $\mathrm{F}_{\text {IS }}$ under assortative mating (0.027) is over six-fold larger than under random mating (-0.005), and of the same order as the standard deviation between replicate ponds, hence detectable in natural populations (Fig.2).

Table 2. Results from population simulations. Simulation results showing mean and standard deviation (in brackets) of expected heterozygosity and $\mathrm{F}_{\mathrm{IS}}$ under random and assortative mating, with different effective population sizes $(\mathrm{Ne})$.

\begin{tabular}{|c|c|c|c|c|}
\hline & \multicolumn{2}{|c|}{ Heterozygosity } & \multicolumn{2}{|l|}{$\mathbf{F}_{\text {IS }}$} \\
\hline & $\mathrm{Ne}=4$ & $\mathrm{Ne}=60$ & $\mathrm{Ne}=4$ & $\mathrm{Ne}=60$ \\
\hline Random Mating & $0.398(0.090)$ & $0.885(0.010)$ & $-0.089(0.205)$ & $-0.005(0.018)$ \\
\hline Assortative Mating & $0.388(0.090)$ & $0.881(0.014)$ & $-0.087(0.210)$ & $0.027(0.025)$ \\
\hline
\end{tabular}




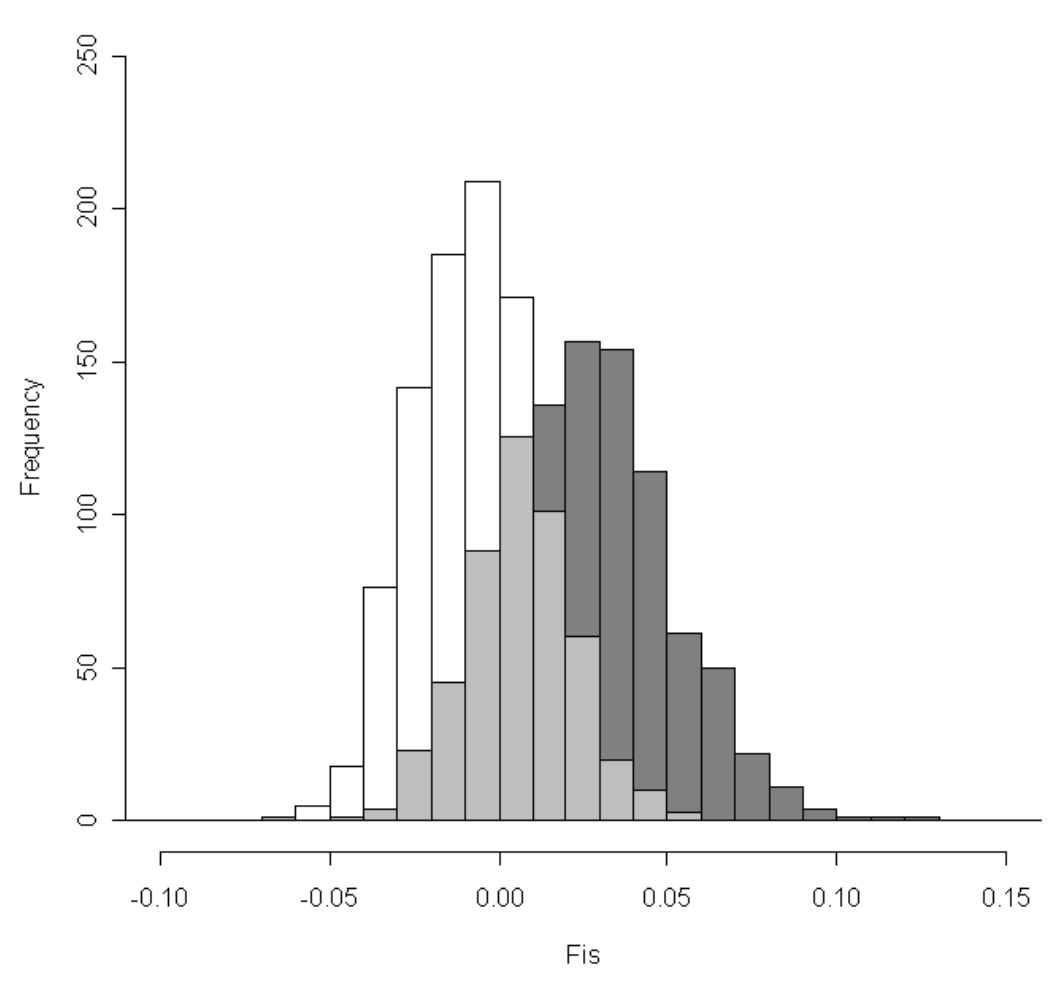

Figure 2. Simulated $F_{\text {IS }}$ under random and assortative mating. Distribution of simulated $F_{I S}$ values under random mating (white) and assortative mating (grey) when $\mathrm{Ne}=60$, demonstrating elevation in $\mathrm{F}_{\mathrm{IS}}$ under assortative mating (the overlap between the distributions is shown in light grey).

\section{Discussion}

The most striking result from this study is the higher $\mathrm{F}_{\mathrm{IS}}$ in $\mathrm{Rv}+$ populations. The $\mathrm{Rv}$ populations have $\mathrm{F}_{\mathrm{IS}}$ levels that are consistent with inbreeding at the level of first cousin or half sibling matings (Frankham et al, 2002). These values (0.068 to 0.102) are comparable to those reported by Palo et al. (2004) (-0.169 to 0.133) in Fennoscandian populations. In $\mathrm{Rv}+$ populations the significantly higher $\mathrm{F}_{\mathrm{IS}}$ is consistent with half sibling, full sibling or parent-offspring matings (Frankham et al, 2002). Another surprising result from this study was the reduction in inbreeding corrected relatedness within $\mathrm{Rv}+$ populations compared to $\mathrm{Rv}$ - populations, i.e. after accounting for the degree of inbreeding, individuals in $\mathrm{Rv}+$ populations are much less related than would be expected. 
I propose that elevated $\mathrm{F}_{\mathrm{IS}}$ and reduced relatedness in $\mathrm{Rv}+$ populations could be explained by assortative mating, whereby uninfected individuals are more likely to mate with other uninfected individuals. The simulations show that even with overlapping generations reduced population size actually lowers $\mathrm{F}_{\mathrm{IS}}$ estimates and hence, cannot explain the elevation of $\mathrm{F}_{\mathrm{IS}}$ in $\mathrm{Rv}+$ populations. Note that $\mathrm{F}_{\mathrm{IS}}$ is a measure of the homozygote excess relative to the local (subpopulation) allele frequencies, so does not reflect the drift of allele frequencies away from the global average, whereas $\mathrm{F}_{\mathrm{ST}}$ would. The simplest way to understand why $\mathrm{F}_{\text {IS }}$ is reduced is to consider rare alleles. In a small population they may occur in only one individual, or be restricted to one sex, and so they would never occur in homozygotes.

Conversely, the simulation demonstrates that assortative mating could explain the elevated $\mathrm{F}_{\mathrm{IS}}$ at neutral loci, even if the assortment is governed by an unlinked locus. Assortment is a violation of the Hardy Weinberg principle, making it more likely that relatives will mate, thus elevating homozygosity over Hardy Weinberg expectations. The effect was not seen in small populations $(\mathrm{Ne}=4)$ due to quick loss or fixation of the assortment allele, and subsequent lack of assortment. However, the analysis of genetic diversity indicates that the populations have not experienced severe genetic drift of this order (see below). It is likely that several loci would be involved in disease resistance (Houle, 1991) and that new genetic variation arising throughout the genome could be recruited, since resistance would be condition dependent. Consequently, disease resistance could be a resilient component of genetic variation, as has been suggested for condition dependent traits involved in sexual selection (Rowe and Houle, 1996).

The assortative mating hypothesis requires the assumption that those escaping Ranavirus infection are more genetically alike than average. Assortative mating could happen if diseased frogs are unable to compete for healthy mates, or by active mate choice. Studies on mate choice in amphibians are limited, however it has been shown to occur in amphibians; for example Marco et al. (1998) showed that male toads preferentially mate with larger females. Amphibians have also been shown to employ mate choice to 
increase their own fitness; hybrid frogs (Rana esculenta) have been shown to preferentially mate with a species that allows them to produce viable offspring (Rana lessonae) rather than mating with their own species, regardless of size or activity (Abt and Reyer, 1993). Moreover, studies have shown that mate choice can be based on an individual's genotype at the Major Histocompatibility Complex; a region which corresponds directly to immunocompetence and thus to disease susceptibility and resistance (Jordan and Bruford, 1998). Another process that could contribute to elevated FIS would be if immigrants to the pond mated assortatively. This trend could be explained if immigrants were infection free because they were yet to be infected, rather than because of their genotype, and if assortative mating is due to poor performance of diseased individuals in obtaining mates, rather than due to active mate choice. This trend could also be explained if immigrants arrived to mate later at a pond, after the majority of the natal individuals had mated. Recall that I sampled the reproductive output as opposed to adults, so the Wahlund effect (Wahlund, 1928) would only result in an increase in $\mathrm{F}_{\mathrm{IS}}$ if migrants mated preferentially with individuals from their original natal ponds. This effect would be of the order of $\mathrm{F}_{\mathrm{ST}}$ if assortment was complete.

$\mathrm{Rv}+$ populations do not show a loss in allelic richness as would be expected for populations suffering such severe bottlenecks (Nei et al, 1975). Simulations show that assortative mating is also expected to further reduce genetic diversity (expected heterozygosity). Overlapping generations does not explain the sustained genetic diversity (expected heterozygosity); simulations showed that if the effective population size falls from 60 to four, $45 \%$ of genetic diversity would be lost over ten generations. I present two hypotheses to explain the sustained allelic diversity in Rv+ populations: (a) allelic richness in $\mathrm{Rv}+$ populations may have been maintained by immigration, or (b) the post-disease populations may remained large enough to have retained their allelic diversity. Outbreaks of Ranavirus are typically characterised by a mass mortality event, and all owners of $\mathrm{Rv}+$ ponds indicated that they had observed a severe reduction in the numbers of frogs in their pond following the initial disease outbreak, thus hypothesis (a) seems more parsimonious. $\mathrm{F}_{\mathrm{ST}}$ does not vary within $\mathrm{Rv}+$ and $\mathrm{Rv}$ - population groups, 
indicating that levels of migration between ponds are comparable within each group. The $\mathrm{F}_{\mathrm{ST}}$ values (approximately 0.1) are comparable with those reported by Palo et al. (2004) who found a range of values from 0.010 to 0.451 in Fennoscandian populations. The expected value of $\mathrm{F}_{\mathrm{ST}}$, the local diversity relative to the global population, depends not on the relative number of immigrants, but the absolute number (Wright, 1950).

\section{Conclusions}

Wild common frog populations that have been exposed to Ranavirus infection show increased $\mathrm{F}_{\mathrm{IS}}$ (excess homozygosity), consistent with increased levels of inbreeding. However, relatedness (corrected for inbreeding) within populations exposed to disease is lower than that in populations that have not been exposed to disease. These findings are best explained by an assortative mating hypothesis, whereby uninfected frogs tend to reproduce with other uninfected frogs. Simulations show that assortment determined by

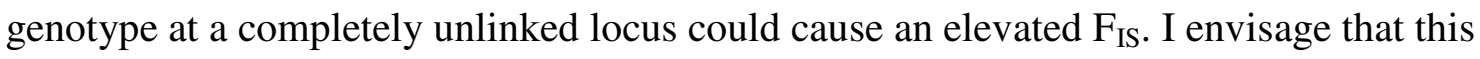
could be a locus involved in disease resistance, and that healthy individuals mate assortatively. No difference in allelic richness was detected between populations exposed to disease and those with no history of disease. Allelic richness could have been maintained by immigration from nearby populations, highlighting the importance of population connectivity. Investigating mate choice at a behavioural (e.g. laboratory studies of mating preferences under different infection statuses) and genetic level (e.g. investigating adaptive loci that are linked to immunity, such as the Major Histocompatibility Complex), would help unravel the effect that this disease has on populations of common frogs. The findings from this study indicate that emerging infectious diseases can affect population dynamics and breeding biology in previously unanticipated ways. 


\title{
CHAPTER 6: Adaptive genetic variation in wild populations of common frogs (Rana temporaria) which have been subject to disease outbreaks (Ranavirus).
} Accepted (December 2008) for publication as:

Teacher, A.G.F., Garner, T.W.J., Nichols, R.A. Evidence for directional selection at a novel major histocompatibility class I marker in wild common frogs (Rana temporaria) exposed to a viral pathogen (Ranavirus). PLOS ONE

\begin{abstract}
Whilst the Major Histocompatibility Complex (MHC) is well characterized in the anuran Xenopus, this region has not previously been studied in another popular model species, the common frog (Rana temporaria). Nor, to date, have there been any studies of MHC in wild amphibian host-pathogen systems. In this chapter, I characterise an MHC class I locus in the common frog, and present primers to amplify both the whole region, and specifically the antigen binding region. As no more than two expressed haplotypes were found in over 400 clones from 66 individuals, it is likely that there is a single class I locus in this species. This finding is consistent with the single class I locus in Xenopus, but contrasts with the multiple loci identified in axolotls, providing evidence that the diversification of MHC class I into multiple loci likely occurred after the Caudata/Anura divergence ( 350 million years ago) but before the Ranidae/Pipidae divergence (approximately 230mya). I use this locus to compare wild populations of common frogs that have been infected with a viral pathogen (Ranavirus) with those that have no history of infection. I demonstrate that certain MHC supertypes are associated with infection status (even after accounting for shared ancestry), and that the diseased populations have more similar supertype frequencies (lower $\mathrm{F}_{\mathrm{ST}}$ ) than the uninfected. These patterns were not seen in a suite of putatively neutral microsatellite loci. I interpret this pattern at the MHC locus to indicate that the disease has imposed selection for particular haplotypes, and hence that common frogs may be adapting to the presence of Ranavirus, which currently kills tens of thousands of amphibians in the UK each year.
\end{abstract}




\section{Introduction}

Many studies searching for the genetic consequences of natural selection have focused their attention on the Major Histocompatibility Complex (MHC) (e.g. Conseguera et al, 2005; Froeschke and Sommer, 2005; Seddon and Ellegren, 2004). The MHC is a group of genes known to be directly involved in the immune response in vertebrates, and variation at the MHC has been linked to disease resistance and susceptibility (e.g. contrasting allelic frequencies in resistant and susceptible chickens with Marek's disease (Briles et al, 1977)). In most vertebrates the MHC comprises several loci which fall into three subgroups, class I, II and III (Hughes and Yeager, 1998). Proteins encoded by the MHC class I and II loci form complexes with antigenic peptides and present these at the cell surface to the T-cell antigen receptors. The class III region encompasses many genes and although some are involved in immunity, they are not directly involved in the presentation of antigens (Campbell and Trowsdale, 1993). MHC class I is primarily involved in presenting endogenously synthesised antigens such as viral proteins, whereas MHC class II is mainly involved in presenting exogenous antigens such as bacteria (Bernatchez and Landry, 2003). However, professional antigen presenting cells (e.g. dendritic cells) can load exogenous antigens onto both class I and II molecules, and autophagy can also deliver endogenous antigens to the class II pathway (Vyas et al, 2008). The class I gene family is divided into the classical class Ia genes and the nonclassical class Ib genes. Class Ia genes are very polymorphic, with most of the polymorphism occurring within the Peptide Binding Region (PBR) which is the area that recognizes antigens (Flajnik et al, 1993). Class Ib genes have limited or no polymorphism and their function remains largely unknown (Flajnik et al, 1993; Ohtsuka et al, 2008).

Expressed MHC loci typically show very high levels of diversity (Piertney and Oliver, 2006). High diversity in MHC loci could have a straightforward benefit, by allowing the identification of a larger number of antigens, and so enabling the organism to cope with a greater range of pathogens. It has therefore been proposed that the high diversity at the 
MHC is maintained by balancing selection, caused by coevolution between hosts and pathogens. One possible form of balancing selection is overdominance (heterozygote advantage), whereby heterozygotes are at an advantage as they can present a broader range of antigens (Doherty and Zinkernagel, 1975). An alternative form is negative frequency-dependent selection, whereby individuals with a novel rare allele have a selective advantage, since pathogens have not evolved to escape their surveillance (Bodmer, 1972; Clarke and Kirby, 1966). Although overdominance and negativefrequency dependent selection are the most frequently proposed modes of selection at the MHC, there are other possibilities. For example, there is evidence for fluctuating selection in TAP genes (Transporter associated with Antigen Processing) in Danish brown trout (Jensen et al, 2008). Fluctuating selection occurs when a heterogeneous environment causes selection for different alleles over time and/or space (Hedrick, 2002). Fluctuating selection could occur if a pathogen is fast evolving, or if different strains occur in different populations. Directional selection can also occur at the MHC, in which case the spread of an advantageous allele would be expected to lead to a loss of genetic variation. Similarly, selection against disadvantageous alleles (negative or purifying selection) would also be expected to reduce diversity. Directional selection may explain why the two human leukocyte antigen (HLA) types associated with protection from malaria are common in West Africans but rare in other racial groups (Hill et al, 1991). Directional selection appears most likely when a single pathogen imposes a substantial proportion of the selection on a particular host species.

In Xenopus laevis there is a single class I locus of the class Ia type (Ohta et al, 2006; Shum et al, 1993), and there is evidence that this locus is involved in susceptibility to viral infection (Ranavirus, family: Iridoviridae) in amphibians. Pre-metamorphic Xenopus tadpoles do not express MHC class Ia genes (Flajnik et al, 1987) and have been shown (in laboratory experiments) to have much higher mortality rates when exposed to Ranavirus than adults which do express these genes (Gantress et al, 2003). Laboratory studies on Xenopus also imply that the MHC genotype may play a role in lethality, with different genotypes conferring differing levels of susceptibility to Ranavirus infection 
(Gantress et al, 2003). Many wild populations of common frogs (Rana temporaria) in the UK have been infected with Ranavirus, which causes skin ulceration, systemic haemmorhaging, and can result in mass mortalities (Cunningham et al, 1996). Ranavirus is estimated to kill tens of thousands of common frogs in the UK each year (Cunningham, 2001).

In this study I characterised MHC class I in the common frog and developed primers to amplify exons 2 and 3 which form the antigen binding region ( $\alpha 1 \& \alpha 2$ domains). I use this novel MHC marker to explore selection in wild populations of common frogs with and without a history of Ranavirus infection. The aims of this study were (1) to identify whether there are specific MHC supertypes associated with infected $(\mathrm{Rv}+)$ and uninfected (Rv-) populations, and thus assess evidence for selection in this system; and (2) to compare MHC diversity with previous information on neutral diversity at microsatellite loci in the same populations.

\section{Materials and Methods}

Sampling

Common frog populations were chosen based on their disease history. Seven populations where chosen which had undergone yearly mortalities caused by Ranavirus for approximately 5 generations (10 years) ( $\mathrm{Rv}+$ populations), and seven populations were chosen which had no history of Ranavirus mortalities (Rv-populations). All populations were located in the South-East of England (Fig. 1) in urban or sub-urban garden ponds. Populations sampled for this study were a subsample of the same populations used in chapter 5. Frogs were captured by hand at each location; each frog was then weighed and held in a clean container at ambient temperature. Frogs below 15 grams were released due to the risks associated with the blood sampling procedures on small animals. Between 5 and 10 frogs of a suitable size were sampled at each location. Each frog was anaesthetised using an aqueous solution of MS222 (Pharmaq Ltd, UK) buffered with $\mathrm{NaHCO}_{3}$ to the $\mathrm{pH}$ of the water where the frog was collected (or at $\mathrm{pH} 7$ if collected on land). Each frog was then bled via cardiocentesis using $0.5 \mathrm{ml}$ heparinised 
insulin syringes, and blood samples of $150 \mu 1$ maximum volume were collected. The frogs were then rinsed in fresh water until conscious, and placed in their original container until all signs of anaesthetic exposure had passed for at least 10 minutes. They were then released back into the wild at the location of capture, taking care to ensure that day-time release did not expose the animals to any additional hazards. Blood samples were centrifuged to pellet the red blood cells, and the pellets were stored in RNALater (Ambion, UK).

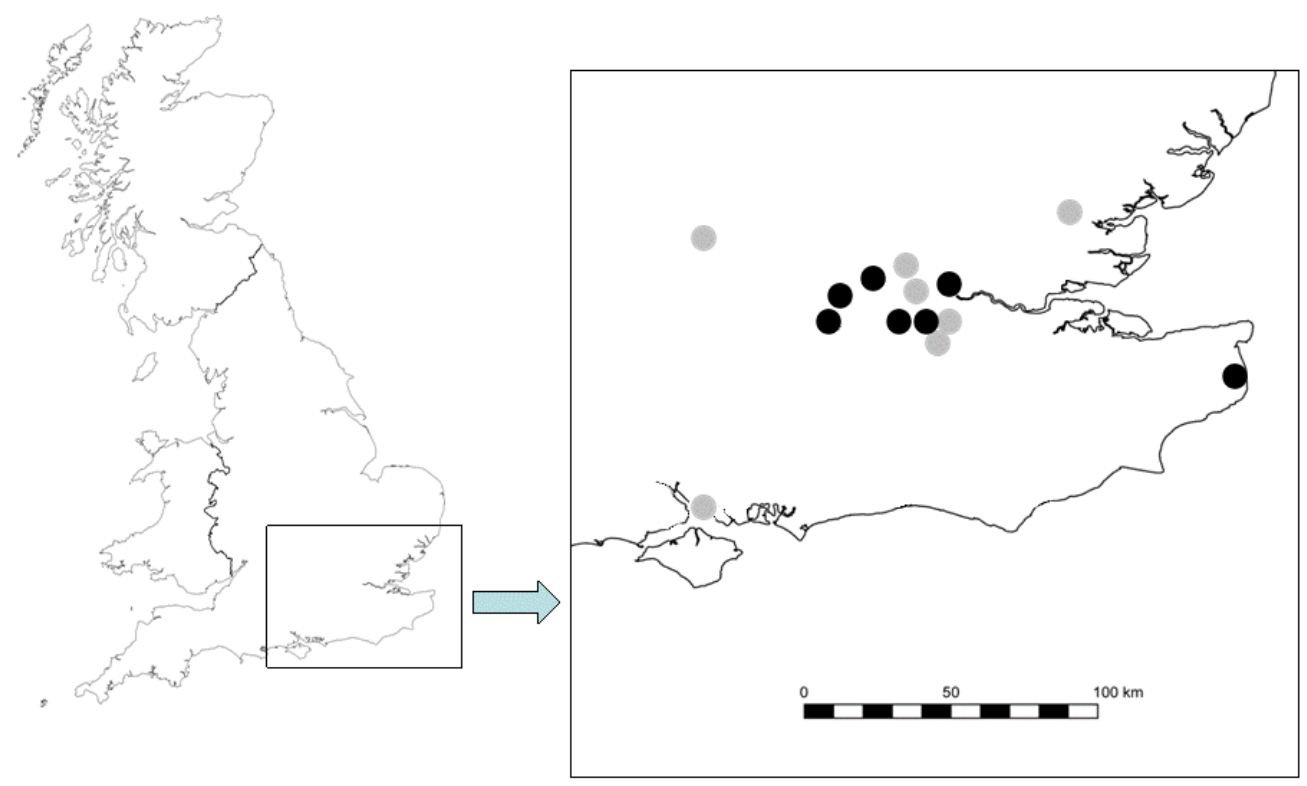

Figure 1. Map showing the location of study sites in England. Infected $(\mathrm{Rv}+)$ populations are indicated by black circles, and uninfected (Rv-) by grey circles.

\section{Molecular techniques}

Total RNA was extracted from blood pellets using the SV 96 Total RNA Isolation System (Promega, UK) which includes DNase treatment to remove genomic DNA (gDNA). The standard protocol for this kit was altered due to the low volumes of blood available; in the first lysis step $40 \mu 1$ blood was added to $300 \mu 1$ RNA Lysis Buffer. RNA concentrations of approximately $0.03 \mu \mathrm{g} / \mathrm{ml}$ were obtained and cDNA synthesis was performed using the First Strand cDNA Synthesis Kit (GE Healthcare/Amersham). 
Published MHC class 1 antigen mRNA sequences from cDNA clones in Rana pipiens (GenBank accession numbers AF185587 and AF185588) were aligned using ClustalW, and Primer3 (Rozen and Skaletsky, 2000) was used to design primers to amplify the whole MHC class I region (approximately 900bp): Rpip9F 5'-

TTCCGACAGTCACACTCTGC-3' and RpipR 5'-GgtGgtCTtGTAGCCTTCTCC-3'. Once Rana temporaria sequences were obtained from these primers, a species-specific reverse primer (RpipR2 5'-TGAAACCCGTACACCAGACA-3') was designed to be used with Rpip9F to amplify only the antigen binding site (exons 2 and 3 corresponding to the $\alpha 1 \& \alpha 2$ domains, approximately $650 \mathrm{bp}$ ). The 650 base-pair region was amplified from cDNA using the Polymerase Chain Reaction (PCR) with primers Rpip9F and RpipR2. The reagents were: $16 \mu 1$ Taq PCR Master Mix (Qiagen, UK), $4 \mu 1$ cDNA, 200pmol of each primer $(2 \mu \mathrm{l})$, and $16 \mu \mathrm{l}$ RNAse-free water. The samples were denatured at $95^{\circ} \mathrm{C}$ for 15 minutes followed by 25 cycles of $94^{\circ} \mathrm{C}$ for $30 \mathrm{sec}, 60^{\circ} \mathrm{C}$ for $1 \min 30 \mathrm{sec}$, and $71^{\circ} \mathrm{C}$ for 2 minutes, followed by a final elongation step of $71^{\circ} \mathrm{C}$ for $10 \mathrm{~min}$ to complete fragment extension. The PCR products were run on $1.2 \%$ agarose gels with $5 \mu$ l loading buffer and a 100 base-pair ladder (Microzone Ltd, UK). The 650 base pair bands were cut from the gels and DNA extracted using the QIAquick Gel Extraction Kit (Qiagen, UK). One sample of gDNA was run with each cDNA PCR plate; gDNA samples yield a 950 base pair band which is not seen in cDNA amplifications, thus confirming that cDNA samples were not contaminated with gDNA.

Ligation and transformation of amplified cDNA products were performed the same day using the Qiagen PCR Cloning Plus Kit (Qiagen, UK). For each individual, 75 $\mu 1$ of transformed cells were spread onto an LB agar plate containing ampicillin, IPTG and Xgal according to the cloning kit instructions. Plates were incubated overnight at $37^{\circ} \mathrm{C}$. Six clonal sequences were required per individual to reduce the chances of missing one allele from a heterozygote to below $\mathrm{p}=0.05$. Seven colonies were picked per individual as colonies did not always contain the insert. The colonies were grown overnight at 
$37^{\circ} \mathrm{C}$, shaking at $225 \mathrm{rpm}$, in individual falcon tubes containing $600 \mu 1 \mathrm{LB}$ broth with $30 \mu \mathrm{g}$ ampicillin. Plasmid purifications were then prepared from the colonies using the Zyppy Plasmid Miniprep Kit (Zymo Research, USA). Purified plasmid DNA (30-50 $\mu \mathrm{g} / \mathrm{ml}$ ) was used for sequencing with standard M13 primers by Cogenics (Essex, UK).

\section{Analysis}

Alleles were confirmed when at least two clones showed identical sequences.

Homozygotes were confirmed on the basis of at least 6 identical sequences; when 6 clones are picked, there is a probability of $0.5^{5}=0.03125$ that there is a second allele which has not been picked by chance. Every confirmed allele was recorded in the data set. Nucleotide sequences (GenBank accession numbers FJ385575 - FJ385703) were aligned using Sequencher v.4. (Gene Codes Corporation), and confirmed as belonging to the MHC Class I conserved domain using BLAST searches (National Center for Biotechnology Information). I used the antigen binding region (comprising of the $\alpha 1$ and $\alpha 2$ domains) for all analyses; this region was identified by amino acid alignment with Xenopus (Flajnik et al, 1999) and Atlantic salmon (Conseguera et al, 2005) sequences.

Observed heterozygosity was calculated within $\mathrm{Rv}+$ and $\mathrm{Rv}$ - groups by dividing the number of observed heterozygotes by the total number of individuals with both alleles typed. Expected heterozygosity was calculated as $H_{E}=1-\sum p_{i}{ }^{2}$ where $p_{\mathrm{i}}$ is the frequency of the $i^{\text {th }}$ allele. The observed number of heterozygotes was compared with Hardy-Weinberg expectations (Frankham et al, 2002) within Rv+ and Rv-groups using Fisher's exact test, and between groups using a Chi-squared test; both tests were implemented in R v.2.4.0. The allelic richness was characterized by the mean number of substitutions per base as calculated by MEGA (Kumar et al, 2001), and compared between Rv+ and Rv-groups using a two-tailed t-test. Modeltest (Posada and Crandall, 1998) was used to test 56 possible DNA substitution model parameters, and identify the best fit model for the data. A neighbour-joining tree was produced using the Tamura-Nei model of nucleotide substitution (Tamura and Nei, 1993), with 100 bootstrap replicates. 
MHC supertypes were identified on the basis of branch length; clusters of individuals $(n>1)$ which were separated from other individuals by a branch length of $\leq 0.007$ (an arbitrarily chosen value, after visual inspection of data) were categorized as a unique supertype (Fig.2). A Chi-squared test was used to compare MHC supertype frequencies between $\mathrm{Rv}+$ and $\mathrm{Rv}$ - populations; however the individuals within each category are not strictly independent, as inhabitants of the same pond are likely to share common ancestry. To overcome this issue, a randomization test was constructed (by Prof Richard A. Nichols, performed in R v.2.4.0, see appendix 4 for source code). The Chi-squared test was repeated (for 10,000 iterations) after reallocating disease status at random to ponds; the source code is included in supporting information. The Chi-squared value from the real data was then compared with the randomized distribution to establish the significance level. For comparison, this method was then repeated with data from 14 putatively neutral microsatellite loci (see chapter 5) collected from the same populations $(\mathrm{Rv}+=77$ individuals, mean per population $=11 ; \mathrm{Rv}-=61$ individuals, mean per population $=9$ ) to investigate whether the $\mathrm{Rv}+$ and $\mathrm{Rv}$ - populations were, by chance, more genetically distinct than average. I used FDist2 (Beaumont and Nichols, 1996) implemented in Lositan (Antao et al, 2008) to further assess the effect of any selection on the variation between populations at the MHC or microsatellite loci. The program compares the $\mathrm{F}_{\mathrm{ST}}$ of marker loci with simulated expected values (conditional on their heterozygosity), and identifies outliers. This method was applied to Rv+ and Rvpopulations individually and combined, using data at the MHC locus, together with data for the same populations from the 14 microsatellite loci to provide information on neutral expectations.

\section{Results}

Full MHC genotypes, based on criteria outlined in the methods section (6+ identical clones for a homozygote, $2+$ clones of each allele for a heterozygote), were obtained for $32 \mathrm{Rv}$ - individuals (7 populations, 4-5 individuals per population) and $31 \mathrm{Rv}+$ individuals (7 populations, 4-5 individuals per population) (see Fig. 1 for sampling locations). Single confirmed alleles were obtained for a further 2 Rv-individuals and 
one Rv+ individual. The 129 alleles from over 400 clones revealed a maximum of two alleles per individual. The MHC region was highly variable, with 178 out of 543 (32.78\%) nucleotides, and 90 out of 181 (49.72\%) amino acids, being polymorphic. The mean proportion of polymorphic nucleotides was significantly greater in the Rv-group compared to the $\mathrm{Rv}+$ group $(\mathrm{Rv}+=0.0793, \mathrm{Rv}-=0.0869, \mathrm{p}<0.0001)$. Heterozygosity in $\mathrm{Rv}+(0.875)$ and $\mathrm{Rv}-(0.936)$ groups did not differ significantly from expected heterozygosity $(\mathrm{Rv}+=0.965, \mathrm{Rv}-=0.972)$, and did not differ significantly between groups.

Modeltest (Posada and Crandall, 1998) identified the TrN $+\mathrm{I}+\mathrm{G}$ model as that which best fitted the data. This model allows for variable base frequencies, equal transversion frequencies and variable transition frequencies, with a proportion of invariable sites $(\mathrm{I}=0.349)$ and a gamma distributed rate variation among sites $(\mathrm{G}=0.248)$ (Tamura and Nei, 1993). A neighbour joining tree of the MHC sequences revealed 23 supertypes (Fig.2). The supertypes differed significantly in frequency between $\mathrm{Rv}+$ and $\mathrm{Rv}$ populations, even after accounting for shared ancestry $\left(\chi^{2}=54.951\right.$, p<0.05) (Fig. 3). The comparable test on fourteen microsatellite loci only identified one additional locus (RtSB3, Berlin et al, 2000) that showed significant (without Bonferroni correction) frequency differences between $\mathrm{Rv}+$ and $\mathrm{Rv}$ - populations $\left(\chi^{2}=79.53, \mathrm{p}<0.05\right)$.

Analysis using Fdist2 (Beaumont and Nichols, 1996) showed that the genetic variation among populations (as measured by $\mathrm{F}_{\mathrm{ST}}$ among $\mathrm{Rv}+$ and $\mathrm{Rv}$ - populations combined) was significantly greater at the MHC locus than the average of the microsatellite loci $\left(\mathrm{F}_{\mathrm{ST}}\right.$ $=0.150, \mathrm{p}<0.03$ ), indicating that this locus may be under some form of selection. Much of this effect was due to variation among the $\mathrm{Rv}$ - populations $\left(\mathrm{F}_{\mathrm{ST}}=0.214, \mathrm{p}<0.008\right)$, whereas the $\mathrm{Rv}+$ populations were genetically more similar in their MHC allele frequencies and not significantly more variable than the microsatellites $\left(\mathrm{F}_{\mathrm{ST}}=0.066\right.$, $\mathrm{p}=0.578$ ). As would be expected from the comparison of frequencies in Rv+ and Rvsites, the microsatellite locus RtSB3 also showed significantly elevated $\mathrm{F}_{\mathrm{ST}}$ in the pooled populations, as did an additional locus RtSB14 (Berlin et al, 2000) - however neither 
showed the difference in $\mathrm{F}_{\mathrm{ST}}$ within $\mathrm{Rv}+$ populations, and only RtSB14 showed this pattern within $\mathrm{Rv}$ - populations.

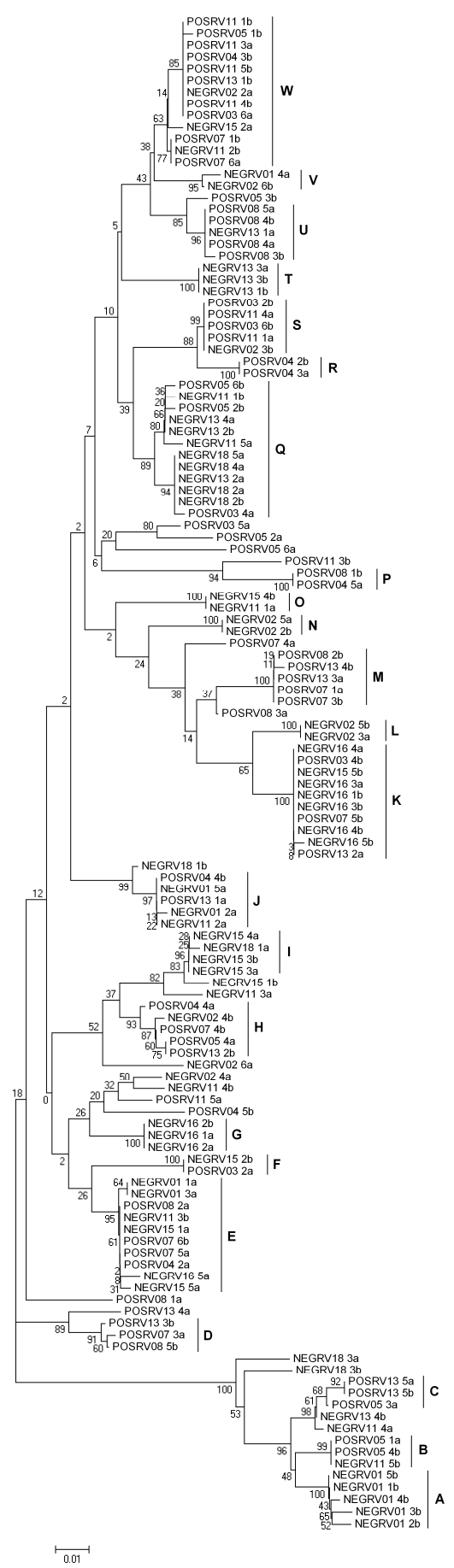

Figure 2. Neighbour-joining tree of MHC sequences with supertypes labelled. Individuals are labelled with disease status 'POS' (Rv+) or 'NEG' (Rv-), population ID, individual ID and allele ID. Bootstrap support values are reported by each node. 


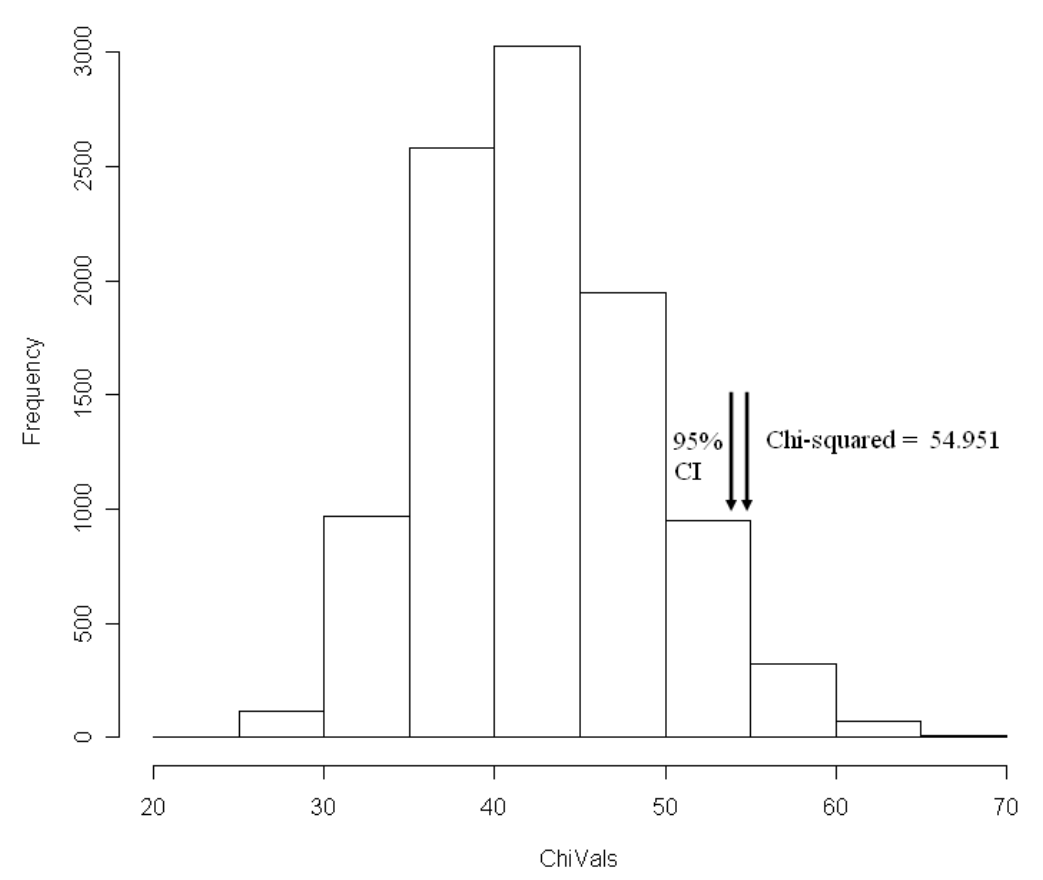

Figure 3. Distribution of Chi-squared values generated by randomising disease status. The Chisquared value obtained from empirical data is marked with an arrow, together with the $95 \%$ confidence limit.

\section{Discussion}

It is apparent from the data that there is a single expressed MHC class I locus in Rana temporaria, as I found a maximum of two alleles per individual ( $\mathrm{n}=66$ individuals). Xenopus species have also been shown to have a single class I locus (Nonaka et al, 1997), in contrast to axolotl (Sammut et al, 1999), indicating that Rana temporaria may have a primordial MHC organization like that of Xenopus. The Caudata (including axolotls) are thought to have diverged from Anura (including Rana and Xenopus sp.) over 350 million years ago, whilst Ranidae (Rana sp.) and Pipidae (Xenopus sp.) diverged approximately 230mya (Roelants et al, 2007). This implies that the diversification of MHC class I into multiple loci occurred between these two dates.

It has previously been shown that MHC Class I deficient tadpoles (this locus is not expressed until metamorphosis) are more susceptible to Ranavirus infection than adults, when exposed to the virus in the laboratory (Gantress et al, 2003; Robert et al, 2005). 
The authors also noted that a particular Xenopus strain showed a high tadpole mortality rate and a slower adult recovery time; suggesting tentatively that the genotype of this strain might confer higher susceptibility, though no sequence information was presented (Gantress et al, 2003). The evidence presented in this chapter comes from a natural experiment in the field, in which some ponds have been exposed to repeated infection for over a decade, whereas others have escaped disease over the same period. I have shown that certain MHC class I supertypes differ in frequency between infected and uninfected wild populations of common frogs, implying directional selection against the alleles conferring greater susceptibility. This finding indicates that the frogs are adapting to the presence of Ranavirus in the wild. Other research has shown that Xenopus is capable of mounting a specific immune response to Ranavirus under laboratory conditions (Maniero et al, 2006); it is possible that this is also occurring in the Rv+ populations in this study.

The reduced allelic richness in $\mathrm{Rv}+$ populations might be explained by a bottleneck due to the frog mortality (Nei et al, 1975). However, I showed in the previous chapter that there was no comparable reduction in allelic diversity at microsatellite loci in infected populations. Hence the reduction in MHC richness appears better explained as another effect of directional selection, which could also produce more uniform allele frequencies (the reduced $\mathrm{F}_{\mathrm{ST}}$ among the $\mathrm{Rv}+$ populations) if the different infected ponds retained similar resistant genotypes. This finding implies that Ranavirus imposes selection for similar genotypes across the geographical range studied, thus there may be little variation in the viral strains affecting these populations. In order to confirm this interpretation, it would be useful to determine the genetic variation of Ranavirus isolates over the same geographical region.

Microsatellite loci RtSB3 and RtSB14 also showed a higher $\mathrm{F}_{\mathrm{ST}}$ than expected under neutrality, though only RtSB3 showed a significant difference between Rv+ and Rvponds under the more stringent randomization test. It is possible that these loci may be linked to an adaptive locus (i.e. had their frequencies changed by genetic hitchhiking, 
see Maynard Smith and Haigh, 1974), potentially even the MHC locus in question. It would be interesting for future studies to try to identify candidate genes by chromosome walking or genome mapping.

Previous microsatellite studies on the same populations (as well as 11 further Rv+ and 9 further Rv-populations) (Chapter 5) provided evidence for assortative mating in Rv+ populations: elevated homozogosity indicated an excess of mating between related individuals. There is therefore an intriguing possibility that assortative mating for resistant MHC haplotypes may be taking place. Experimental studies of resistance/susceptibility of MHC genotypes under deliberate Ranavirus infection, and associated mate choice could prove fruitful for understanding the mechanism fully.

\section{Conclusions}

Rana temporaria appears to have a single expressed MHC class I locus, as has been reported for Xenopus, but in contrast to axolotls. By comparing wild common frog populations that are infected and uninfected with Ranavirus, we demonstrated that certain MHC supertypes are associated with populations of each disease status. Reduced genetic diversity was detected in infected populations, which may either be caused by population bottlenecks, or by directional selection for particular supertypes. Infected populations also show more uniform allele frequencies (reduced $\mathrm{F}_{\mathrm{ST}}$ compared to uninfected populations), implying that there may be selection for similar resistant genotypes across these populations. This preliminary evidence for directional selection under infection may indicate that wild populations of common frogs in the UK are adapting to the presence of Ranavirus. 


\section{Chapter 7: Discussion}

This thesis has focused on understanding the impact of major events on the genetic patterns within and between populations. I have explored the legacy that the Pleistocene glaciations have left, and investigated the consequences of recent disease outbreaks on populations of common frogs. This discussion provides a brief synthesis of the results from the previous chapters, with an emphasis on the broader picture, including the conservation implications resulting from this research, and potential further studies.

\section{Glaciation and range shifts}

The Pleistocene European glaciations represented a major crisis for temperate species, drastically reducing the habitable land to the warmer regions such as Iberia, Italy and the Balkans (Hewitt, 2000; Hewitt, 2004; Malez, 1972; Taberlet et al, 1998). Since the ice melted approximately 10,000 years ago, species that were confined to glacial refuges were able to re-populate Europe (Taberlet et al, 1998; Webb and Bartlein, 1992). Substantial divergence between Eastern and Western European common frog mtDNA haplotypes has previously been identified by Palo et al. (2004), likely representing two colonisation events from an Iberian and a Balkan or Italian refugium. These findings were mirrored in the data shown in Chapter 2; however the phylogenetic trees presented in this thesis also revealed a previously undiscovered overlap between the two lineages in Southern Europe. It is likely that the Eastern colonisation event primarily moved northwards, but that some individuals moved West, channeled by the Alps into the South of France and the North of Spain.

As more detailed phylogenetic studies are completed, it is becoming apparent that postglacial colonisation routes are often more complicated than previously assumed, and that smaller, cryptic refugia may have existed as well as the larger Southern European refugia (Steward and Lister, 2001). There has previously been some genetic and palaeontological evidence for a small refugium in the South of Ireland, which is thought to have remained ice-free, and may have supported some cold-tolerant species (Forbes, 
1846; Hoarau et al, 2007; Rowe et al, 2006; Yalden, 1999). The findings from this thesis provide support to the existence of this refugium, as novel mitochondrial DNA haplotypes were found in common frogs from the South and West of Ireland that were not found elsewhere in Europe. Ireland is unusual in terms of the species that inhabit it, and their colonisation history. Several species are only found in Ireland and Iberia, and some have suggested that these species may have been moved by humans along a trade route between these regions (Corbet, 1961; Corbet, 1962; Mascheretti et al, 2003; Mitchell, 1986; O'Rourke, 1970; Platts and Speight, 1988; Praeger, 1939), though there is no evidence for this having occurred in common frogs. There is also some evidence (primarily based on sea-level modelling) for a land-bridge between Ireland and England, across which some species could have migrated; however there is scant evidence to directly support such movements (Devoy, 1985; Lambeck, 1996; Lambeck and Purcell, 2001; Preece et al, 1986; Wingfield, 1995). My data provide indirect support for such a land bridge. As well as identifying unique mtDNA haplotypes in Ireland, I also showed that some Irish common frogs belong to a widespread Western European haplotype. This finding indicates that a secondary colonisation event may have occurred into Ireland, following a Western European expansion. It is probable that this Western expansion would have reached Ireland via Britain, as Britain is the closest land mass to Ireland.

Chapter 3 further examined this scenario by sub-sampling populations in the East of Ireland and South-East of England. The mtDNA haplotypes found in these regions are indistinguishable, however when microsatellites (faster evolving markers) are used, distinct differences appear between the two regions. Frog populations in the East of Ireland showed significantly reduced genetic diversity compared to the English populations and, for the most part, the alleles present in the Irish populations were a subset of the English alleles. Using correspondence analysis, I demonstrated that these populations can be clearly distinguished based on allelic frequencies at microsatellite loci. These findings are consistent with the colonisation of Ireland via England or Western Europe, as the associated founder events would be expected to result in a loss of genetic diversity (Nei et al, 1975; Nichols and Hewitt, 1994). 


\section{Implications}

Genetic differences below the species level have previously been used to identify priorities for conservation. Variation in mtDNA is sometimes used to define Evolutionary Significant Units (ESUs), which aim to conserve evolutionary heritage (Moritz, 1994). For example mtDNA studies showed that the African elephant actually consists of two separate subspecies, each with a different IUCN threat status (Forest elephant: endangered; Savannah elephant: vulnerable) (Roca et al, 2001). An alternative approach is to attempt to conserve current population units (termed Management Units, or MUs), regardless of their evolutionary distinctiveness (Moritz, 1994). For example, there is currently a large, EU-funded project that is using this method for managing salmon fishing at sea to ensure that no one river population is over-exploited (SALSEAMerge, EU contract no. 212529). However there has been much debate about the practicalities of such approaches (especially the ESU approach), as both can miss important aspects of population distinctiveness, such as genetic variation that is of adaptive significance (cf. Crandall et al, 2000; Moritz, 1994).

In Chapter 1, European common frogs were divided into mtDNA lineages; however these lineages likely represent different ancestral histories rather than adaptive differences. Although interesting, it is debatable how relevant these divisions would be in management terms. Mitochondrial DNA also indicated that there was little difference between common frogs in England and eastern Ireland, however, microsatellites revealed much lower genetic diversity in the Irish populations. Reduced genetic diversity has been linked to increased vulnerability, for example Italian agile frog (Rana latastei) populations with reduced genetic diversity experience higher mortality rates when exposed to Ranavirus (Pearman and Garner, 2005). In this way, mtDNA studies may be able point the direction to geographical regions which are likely to have reduced genetic diversity due to founder events, and thus potentially be more at risk from events such as infectious disease outbreaks. However caution must be applied, as again, a reduction in genetic diversity at neutral markers may not reflect the adaptive potential of a population. 


\section{Infectious disease}

Disease has been named by the International Union for Conservation of Nature as a major threat to amphibians worldwide (IUCN, 2004). Outbreaks of the fungal disease, chytridiomycosis, are thought to have lead to species declines and even the extinction of the golden toad (Bufo periglenes) (Pounds et al, 2006). A less extensively studied disease of amphibians, Ranavirus, causes mass mortalities of various species including common frogs in the UK, but has not as yet been linked to population declines (Cunningham et al, 1996; Green et al, 2002). By searching archived records of Ranavirus-consistent mortality events, and confirming disease status in the laboratory, I identified a number of populations which seem to experience recurrent mortality, with deaths occurring every year or two. Information of population sizes before, and ten years after the mass mortality event indicate that these populations have experienced approximately $80 \%$ declines in the numbers of frogs present. Futhermore, a Generalised Linear Model indicated that the variable most strongly associated with these population size changes was disease status.

Surprisingly, despite these large population declines, infected populations showed no reduction in genetic diversity (allelic richness) at microsatellite loci. It is probable that diversity in these populations is being maintained by low levels of immigration from nearby populations, indicating that connectivity between populations may be important for this species. The most striking result was that infected populations show significantly elevated homozygosity (indicating an excess of mating between related individuals), however these populations also showed lower relatedness. At first these results seem contradictory, but they are explicable by assortative mating, whereby healthy individuals tend to mate with other healthy individuals. This explanation requires that those escaping Ranavirus infection are more genetically alike than average. Assortative mating could either occur through active mate-choice (e.g. for healthy individuals), or by infected individuals being unable to compete for healthy mates. A further possibility is that disease-free immigrants mate assortatively, either through one of the mechanisms already mentioned, or if the immigrants arrive later at the pond after the majority of the 
natal frogs have mated. Population simulations indicated that these patterns could not be explained by population size reductions, but could indeed be explained by assortative mating (even if a mate choice locus is unlinked to the genetic markers).

In order to examine this further, I developed a new marker at the Major Histocompatibility Complex (MHC) Class I locus; a region which is known to be directly involved in the immune response to viral pathogens (e.g. Hughes and Yeager, 1998). This marker proved fruitful, as the frequencies of supertypes varied with infection status, even after accounting for shared ancestry. Infected populations also showed reduced genetic diversity, and more uniform allele frequencies, providing further evidence for directional selection for the more resistant MHC genotypes. Although many studies indicate that genetic diversity at the MHC is maintained by balancing selection, in situations such as this where a large proportion of selection is posed by a single pathogen, directional selection for resistance alleles or against susceptibility alleles is inevitable. Interestingly, the MHC has also been shown to be involved in mate-choice in a broad range of species (Jordan and Bruford, 1998). If certain alleles are associated with resistance, then mate choice for a resistant MHC type could potentially provide a mechanism for such assortative mating.

\section{Implications}

Ranavirus is thought to kill tens of thousands of common frogs each year in the UK, and although it appears that this pathogen is capable of causing localised population declines, it seems likely that common frogs are adapting to cope with infection. Even though these results suggest that Ranavirus may not be a direct conservation issue for common frogs, it remains a serious welfare issue due to the severity of the symptoms experienced by the animals. Because of this, care should be taken not to introduce infections to the wild. Evidence from other research points towards imported pet goldfish or bullfrogs as a means for the introduction of Ranavirus to ponds, and so exotic species should not be added to garden ponds (Cunningham, 2001). Further, translocation of common frogs (adults, tadpoles or eggs) between ponds may be another 
route for transmission. Translocations, species introductions, and the pet trade are all regulated to some degree in the UK (though seldom for wild native species); however they are rarely considered with respect to the spread of infectious disease. Assessing the risks of wildlife diseases is imperative for the development of successful management policies (see Appendix 5, which is a Parliamentary Office of Science \& Technology note that I produced on a NERC-funded placement as part of this $\mathrm{PhD}$ ).

The unforeseen findings that disease may influence the optimal mating behaviour, and that genetic diversity may be maintained by immigration, imply that the effects of emerging infectious diseases can be wider than might be expected. The majority of studies of the impacts of infectious diseases look at the effects on population numbers, or on basic genetic diversity, and so may be missing the more cryptic changes in population dynamics. In order to truly assess the impact of a disease, future researchers should consider broader ranging studies, ideally incorporating information on behaviour, ecology, immunology and genetics for both the affected populations as well as comparable unaffected populations.

\section{Directions for future investigations}

It would be interesting to extend sampling coverage of Ireland for microsatellite studies in order to see how the genetic variation differs across the ranges of the different mtDNA haplotypes. Extending the microsatellite analysis to include samples from Europe could also give a clearer picture of the postglacial colonisation routes into England and Ireland. Establishing whether reduced population-level genetic diversity leads to an increased susceptibility to Ranavirus in Rana temporaria would be valuable for assessing the risk should this disease reach Ireland.

Laboratory studies of mating preference under differing infection statuses could be used to confirm whether assortative mating occurs. In particular it would be interesting to establish whether having a mix of infected and uninfected individuals within a population leads to assortative mating. Experimental studies of the 
resistance/susceptibility of different MHC genotypes under Ranavirus infection could reveal the genomic sequences that confer resistance. In addition, laboratory studies of assortative mating could be extended to compare the mating preferences of individuals with different MHC genotypes.

Further to comparing populations with no history of disease to those with a history of recurrent disease, it would also be useful to investigate populations that experienced no further mortalities after their initial mass mortality event. Have these populations developed immunity? Specific antibodies to Ranavirus have been found in Xenopus (Maniero et al, 2006); it would be fascinating to see whether such antibodies were present in the common frog populations used in this study, and whether common frogs are capable of mounting an immune response that confers long-term protection. If common frogs can mount an immune response, it would be interesting to see how quickly immunity develops, how complete this immunity would be, and whether factors such as pollution and climate could compromise the immune system. 


\section{References}

Abdelkrim J, Pascal M, Samadi S (2005). Island colonization and founder effects: the invasion of the Guadeloupe islands by ship rats (Rattus rattus). Molecular Ecology 14(10): 2923-2931.

Abt G, Reyer H-U (1993). Mate choice and fitness in a hybrid frog: Rana esculenta femailes prefer Rana lessonae males over their own. Behavioural Ecology and Sociobiology 32: 221-228.

Acevedo-Whitehouse K, Gulland F, Greig D, Amos W (2003). Disease susceptibility in Californian sea lions. Nature 422: 35.

Aidoo M, Terlouw D, Kolczak M, McElroy P, ter Kuile F, Kariuki S et al (2002). Protective effects of the sickle cell gene against malaria morbidity and mortality. The Lancet 359(9314): 1311 - 1312.

Alexandrino J, Froufe E, Arntzen JW, Ferrand N (2001). Genetic subdivision, glacial refugia and postglacial recolonization in the golden-striped salamander, Chioglossa lusitanica (Amphibia: Urodela) Molecular Ecology 9(6): 771-781.

Aljanabi SM, Martinez I (1997). Universal and rapid salt-extraction of high quality genomic DNA for PCR-based techniques. Nucleic Acids Research 25(22): 4692-4693.

Allard RW ( 1990). The genetics of host-pathogen coevolution: Implications for genetic resource conservation. The Journal of Heredity 81(1): 1-6.

Anderson RM, May RM (1979a). Population biology of infectious diseases part I. Nature 280: 361-367.

Anderson RM, May RM (1979b). Population biology of infectious diseases part II. Nature 280: 455-461.

Antao T, Lopes A, Lopes RJ, Beja-Pereira A, Luikart G (2008). LOSITAN: A workbench to detect molecular adaptation based on a Fst-outlier method. BMC Bioinformatics 9: 323.

Avise J (1994). Molecular Markers, Natural History and Evolution. Chapman and Hall: New York.

Avise JC (ed) (2000). Phylogeography: The history and formation of species. Harvard University Press: Cambridge, Massachusetts and London, England. 
Babik W, Branicki W, Sandera M, Litvinchuk S, Borkin LJ, Irwin JT et al (2004). Mitochondrial phylogeography of the moor frog, Rana arvalis. Molecular Ecology 13: 1469-1480.

Baker JMR, Halliday T (1999). Amphibian colonization of new ponds in an agricultural landscape. Herpetological Journal 9: 55-63.

Barone M, Frank T (2003). Habitat age increases reproduction and nutritional condition in a generalist arthropod predator Oecologia 135(1): 78-83.

Beaumont MA, Nichols RA (1996). Evaluating loci for use in the genetic analysis of population structure. Proceedings of the Royal Society of London, B Biological Science 263: $1619-1626$.

Begon M, Harper JL, Townsend CR (1996). Ecology. Blackwell: Oxford.

Belkhir K, Borsa P, Chikhi L, Raufaste N, Bonhomme F. (1996). Laboratoire Génome, Populations, Interactions, CNRS UMR 5000, Université de Montpellier II: Montpellier.

Bellinger MR, Johnson JA, Toepfer J, Dunn P (2003). Loss of genetic variation in Greater Prairie Chickens following a population bottleneck in Wisconsin, U.S.A. Conservation Biology 17(3): 717-724.

Berlin S, Merila J, Ellegren H (2000). Isolation and characterization of polymorphic microsatellite loci in the common frog, Rana temporaria. Molecular Ecology 9: 19381939.

Bernatchez L, Landry C (2003). MHC studies in nonmodel vertebrates: what have we learned about natural selection in 15 years? Journal of Evolutionary Biology 16: 363377.

Blab J (ed) (1986). Biologie, Ökologie und Schutz von Amphibien Kilda-Verlag: Bonn.

Bodmer WF (1972). Evolutionary significance of the HL-A system. Nature 237: 139183.

Bollinger TK, Mao J, Schock D, Bringham RM, Chinchar VG (1999). Pathology, isolation and preliminary characterisation of a novel iridovirus from tiger salamanders in Saskatchewan. Journal of Wildlife Diseases 35: 413-429.

Bonin A, Bellemain E, Bronken Eidesen P, Pompanon F, Brochmann C, Taberlet P (2004). How to track and assess genotyping errors in population genetics studies. Molecular Ecology 13: 3261-3273. 
Bowcock AM, Ruiz-Linares A, Tomfohrde J, Minch E, Kidd JR, Cavalli-Sforza LL (1984). High resolution of human evolutionary trees with polymorphic microsatellites. Nature 368: 455-457.

Boyle DG, Boyle DB, Olsen V, Morgan JAT, Hyatt AD (2004). Rapid quantitative detection of chytridiomycosis (Batrachochytrium dendrobatidis) in amphibian samples using real-time Taqman PCR assay. Diseases of Aquatic Organisms 60: 141-148.

Briles WE, Stone HE, Cole RK (1977). Marek's disease: effects of B histocompatibility alloalleles in resistant and susceptible chickens. Science 195: 193-195.

Brunner JL, Schock DM, Davidson EW, Collins JP (2004). Intraspecific reservoirs: Complex life history and the persistence of a lethal Ranavirus. Ecology 85: 560-566.

Campbell RD, Trowsdale J (1993). Map of the human MHC. Immunology Today 14: 349-352.

Carey C, Cohen N, Rollins-Smith L (1999). Amphibian declines: an immunological perspective. Developmental and comparative immunology 23: 459-472.

Charlesworth B, Morgan MT, Charlesworth D (1993). The effect of deleterious mutations on neutral molecular variation. Genetics 134: 1289-1303.

Charlesworth D, Charlesworth B (1987). Inbreeding depression and its evolutionary consequences. Annual Review of Ecology and Systematics 18: 237-268.

Chinchar VG (2002). Ranaviruses (family Iridoviridae): emerging cold-blooded killers. Achives of Virology 147: 447-470.

Chinchar VG, Wang J, Murti G, Carey C, Rollins-Smith L (2001). Inactivation of frog virus 3 and channel catfish virus by esculentin-2P and ranatuerin-2P, two antimicrobial peptides isolated from frog skin. Virology 288: 351-357.

Clarke BC, Kirby DRS (1966). Maintenance of histocompatibility polymorphism. Nature 211: 999-1000.

Clement M, Posada D, Crandall K (2000). TCS: a computer program to estimate gene genealogies. Molecular Ecology 9(10): 1657-1660.

Cohan FM (1984). Can uniform selection retard random genetic divergence between isolated conspecific populations? Evolution 38: 495-504.

Coltman DW, Pilkington JG, Smith JA, Pemberton JM (1999). Parasite-mediated selection against inbred Soay sheep in a free-living, island population. Evolution 53(4): 1259-1267. 
Comeron JM, Williford A, Kliman RM (2008). The Hill-Robertson effect: evolutionary consequences of weak selection and linkage in finite populations. Heredity 100: 19-31.

Conseguera S, Megens HJ, Leon K, Stet RJM, Jordan WC (2005). Patterns of variability at the major histocompatibility class II alpha locus in Atlantic salmon contrast with those at the class I locus. Immunogenetics 57: 16-24.

Corbet GB (1961). Origin of the British insular races of small mammals and of the 'Lusitanian' fauna. Nature 191: 1037-1040.

Corbet GB (1962). The Lusitanian element in the British fauna. Science Progress 50: 177-191.

Crandall KA, Bininda-Emonda ORP, Mace GM, Wayne RK (2000). Considering evolutionary processes in conservation biology. Trends in Ecology and Evoltution 15(7): 290-295.

Cunningham AA (2001). Investigations into mass mortalities of the common frog (Rana temporaria) in Britain: epidemiology and aetiology. PhD thesis, University of London.

Cunningham AA, Daszak P (1998). Extinction of a species of land snail due to infection with a microsporidian parasite. Conservation Biology 12(5): 1139-1141.

Cunningham AA, Daszak P, Rodriguez JP (2003). Pathogen pollution: defining a parasitological threat to biodiversity conservation. Journal of Parasitology 89: S78-S83.

Cunningham AA, Langton TES, Bennett PM, Lewin JF, Drury SEN, Gough RE et al (1996). Pathological and microbiological findings from incidents of unusual mortality of the common frog (Rana temporaria). Philosophical Transactions of the Royal Society of London, Series B 351: 1539-1557.

Daszak P, Berger L, Cunningham AA, Hyatt AD, Green DE, Speare R (1999). Emerging infectious diseases and amphibian population declines. Emerging Infectious Diseases 5: 735-748.

Daszak P, Cunningham AA, Hyatt AD (2003). Infectious disease and amphibian population declines. Diversity and Distributions 9: 141-150.

Devoy RJ (1985). The problem of a late Quaternary landbridge between Britain and Ireland. Quaternary Science Review 4: 43-58.

Doherty PC, Zinkernagel RM (1975). Enhanced immunological surveillance in mice hererozygous at the H-2 gene complex. Nature 256: 50-52. 
Dumolin-Lapegue S, Demesure B, Fineschi S, Corre VL, Petit RJ (1997).

Phylogeographic structure of white oaks throughout the European continent. Genetics

146: $1475-1487$.

Elmberg J (1987). Random mating in a boreal population of European common frogs Rana temporaria. Ecography 10(3): 193-195.

Elmberg J (1991). Factors affecting male yearly mating success in the common frog, Rana temporaria Behavioural Ecology and Sociobiology 28(2): 125-131.

England PR, Osler GHR, Woodworth LM, Montgomery ME, Briscoe DA, Frankham R (2003). Effects of intense versus diffuse population bottlenecks on microsatellite genetic diversity and evolutionary potential. Conservation Genetics 4: 595-604.

Entwistle PF, Adams PHW, Evans HF, Rivers CF (1983). Epixootiology of a nuclear polyhedrosis virus (Baculoviridae) in European spruce sawfly (Gilpinia hercyniae): Spread of disease from small epicentres in comparison with spread of Baculovirus diseases in other hosts. The Journal of Applied Ecology 20(2): 473-487.

Ersts PJ. (2008). American Museum of Natural History, Center for Biodiversity and Conservation. Available from http://biodiversityinformatics.amnh.org/open_source/gdmg.

Felsenstein J (1989). PHYLIP - Phylogeny Inference Package (Version 3.2). Cladistics 5: 164-166.

Flajnik MF, Hsu E, Kaufman JF, Du Pasquier L (1987). Changes in the immune system during metomorphosis of Xenopus. Immunology Today 8: 58-64.

Flajnik MF, Kasahara M, Shum BP, Salter-Cid L, Taylor E, Du Pasquier L (1993). A novel type of class I gene organization in vertebrates: a large family of non-MHC-linked class I genes is expressed at the RNA level in the amphibian Xenopus. The EMBO Journal 12(11): 4385-4396.

Flajnik MF, Ohta Y, Greenberg AS, Salter-Cid L, Carrizosa A, Du Pasquier L et al (1999). Two ancient allelic lineages at the single classical class I locus in the Xenopus MHC. The Journal of Immunology 163(3826-3833).

Forbes E (1846). On the connexion between the distribution of the existing fauna and flora of the British Isles, and the geographical changes which have affected their area, especially during the epoch of northern drift. Memoirs of the Geological Survey of Great Britain 1: 336-432.

Frankham R, Ballou JD, Briscoe DA (2002). Introduction to Conservation Genetics. Cambridge University Press: Cambridge, UK. 
Froeschke G, Sommer S (2005). MHC class II DRB variability and parasite load in the striped mouse (Rhabdomys pumilio) in the Southern Kalahari. Molecular Biology and Evolution 22(5): 1254-1259.

Gantress J, Maniero GD, Cohen N, Robert J (2003). Development and characterization of a model system to study amphibian immune responses to iridoviruses. Virology 311: 254-262.

Garner TWJ, Pearman PB, Angelone S (2004). Genetic diversity across a vertebrate species' range: a test of the central-peripheral hypothesis. Molecular Ecology 13: 10471053.

Garner TWJ, Tomio G (2001). Microsatellites for use in studies of the Italian agile frog, Rana latastei. Conservation Genetics 2: 77-80.

Gasc J-P, Cabela A, Crnobrnja-Isailovic J, Dolmen D, Grossenbacher K, Haffner P et al (eds) (1997). Atlas of amphibians and reptiles in Europe. Societas Europaea

Herpetological and Museum National D'Histoire Naturelle: Paris, pp 496.

Goldstein DB, Linares AR, Cavalli-Sforza LL, Feldman MW (1995). An evaluation of genetic distances for use with microsatellites. Genetics 139: 463-471.

Gosner KL (1960). A simplified table for staging anuran embryos and larvae with notes on identification. Herpetologica 16: 183-190.

Goudet J (1995). Fstat version 1.2: a computer program to calculate F statistics. Journal of Heredity 86(6): 485-486.

Green DE, Converse KA, Schrader AK (2002). Epizootiology of sixty-four amphibian morbidity and mortality events in the USA, 1996-2001. Annals New York Academy of Sciences 969: 323-339.

Guindon S, Lethiec F, Duroux P, Gascuel O (2005). PHYML Online--a web server for fast maximum likelihood-based phylogenetic inference. Nucleic Acids Research 33((Web Server issue):W557-9).

Haapanen A (1982). Breeding of the common frog (Rana temporaria L.). Annales Zoologici Fennici 19: 75-79.

Hamill RM, Doyle D, Duke EJ (2006). Spatial patterns of genetic diversity across European subspecies of the mountain hare, Lepus timidus L. Heredity 97: 355 - 365. 
Hannu S, Mäkinen HS, Shikano T, Cano JM, Merilä J (2008). Hitchhiking mapping reveals a candidate genomic region for natural selection in three-spined stickleback chromosome VIII. Genetics 178(1): 453-465.

Hasegawa M, Kishino H, Yano T (1985). Dating of the human-ape splitting by a molecular clock of mitochondrial DNA. Journal of Molecular Evolution 22: 160-174.

Hedrick PW (2002). Pathogen resistance and genetic variation at MHC loci. Evolution 56: $1902-1908$.

Hewitt G (2000). The genetic legacy of the Quaternary ice ages. Nature 405: 907-913.

Hewitt GM (2004). Genetic consequences of climatic oscillations in the Quaternary. Philos Trans R Soc Lond Ser B-Biol Sci 359: 183-195.

Hill AVS, Allsopp CEM, Kwiatkowski D, Anstey NM, Twumasi P, Rowe PA et al (1991). Common West African HLA antigens are associated with protection from severe malaria. Nature 352: 595-600.

Hill WG, Robertson A (1966). The effect of linkage on limits to artificial selection. Genetical Research 8: 269-294.

Hoarau G, Coyer JA, Veldsink JG, Stam WT, Olsen JL (2007). Glacial refugia and recolonization pathways in the brown seaweed Fucus serratus. Molecular Ecology 16(17): 3606-3616.

Hofman S, Spolsky C, Uzzell T, Cogălniceanu D, Babik W, Szymura JM (2007). Phylogeography of the fire-bellied toads Bombina: independent Pleistocene histories inferred from mitochondrial genomes. Molecular Ecology 16: 2301-2316.

Holder M, Lewis PO (2003). Phylogenetic estimation: traditional and bayesian approaches. Nature Reviews Genetics 4: 275-284.

Houlahan JE, Findlay CS, Schmidt BR, Meyer AH, Kuzmin SL (2000). Quantitative evidence for global amphibian population declines. Nature 404: 752-755.

Houle D (1991). Genetic covariance of fitness correlates: what genetic correlations are made of and why it matters. Evolution 45: 30-648.

Huelsenbeck JP, Ronquist F (2001). MRBAYES: Bayesian inference of phylogeny. Bioinformatics 17: 754-755.

Hughes AL, Nei M (1988). Pattern of nucleotide substitution at major histocompatibility complex class I loci reveals overdominant selection. Nature 335: 167-170. 
Hughes AL, Yeager M (1998). Natural selection at major histocompatibility complex loci of vertebrates. Annual Review of Genetics 32: 415-435.

Hyatt AD, Gould AR, Zupanovic Z, Cunningham AA, Hengstberger S, Whittington RJ et al (2000). Comparative studies of piscine and amphibian iridoviruses. Archives of Virology 145: 301-331.

IUCN. (2004). Vol. 2006. IUCN, Conservation International, Natureserve.

Jancovich JK, Davidson EW, Morado JF, Jacobs BL, Collins JP (1997). Isolation of a lethal virus from the endangered salamander Ambystoma tigrinum stebbinsi. Diseases of Aquatic Organisms 31: 161-167.

Jensen LF, Hansen MM, Mensberg K-LD, V. L (2008). Spatially and temporally fluctuating selection at non-MHC immune genes: evidence from TAP polymorphisms in populations of brown trout (Salmo trutta, L.). Heredity 100(1): 79-91.

Jordan WC, Bruford MW (1998). New perspectives on mate choice and the MHC. Heredity 81: 127-133.

Kanchanakhan S (1998). An ulcerative disease of the cultured tiger frog, Rana tigrina, in Thailand. AAHRI Newsletter 7: 1-2.

Kashi Y, Soller M (1999). Functional roles of microsatellites and minisatellites. In: Goldstein DB and Schlotterer C (eds) Microsatellites: Evolution and Applications. Oxford University Press Inc.: New York, pp 10-22.

Koeslag JH (2003). Evolution of cooperation: cooperation defeats defection in the cornfield model. Journal of Theoretical Biology 224: 399-410.

Kumar S, Tamura K, Jakobsen IB, Nei M. (2001). Arizona State University: Tempe, Ariz.

Lambeck K (1996). Glaciation and sea-level change for Ireland and the Irish Sea since Late Devensian/Midlandian time. Journal of the Geological Society 153(6): 853-872.

Lambeck K, Purcell AP (2001). Sea-level change in the Irish Sea since the last glacial maximum: constraints from isostatic modelling. Journal of Quaternary Science 16: 497 506.

Lambert DM, Ritchie PA, Millar CD, Holland B, Drummond AJ, Baroni C (2002). Rates of evolution in ancient DNA from adélie penguins. Science 295: 2270-2273.

Leberg PL (1992). Effects of population bottlenecks on genetic diversity as emasured by allozyme electrophoresis. Evolution 46: 477-494. 
Malez M (1972). On the distribution of ice-age animals in the late Pleistocene of SouthEastern Europe. Radovi Jugoslavenske Akademija Znanosit i Umjetnosti 364: 133-180.

Maniero GD, Morales H, Gantress J, Robert J (2006). Generation of a long-lasting, protective, and neutralizing antibody response to the ranavirus FV3 by the frog Xenopus. Developmental and Comparative Immunology 30(7): 649-657.

Mantel N (1967). The detection of disease clustering and a generalized regression approach. Cancer Research 27: 209-220.

Mao J, Tham TN, Gentry GA, Aubertin A, Chinchar VG (1996). Cloning, Sequence Analysis, and Expression of the Major Capsid Protein of the Iridovirus Frog Virus 3 Virology 216(2): 431-436.

Marco A, Kiesecker JM, Chivers DP, Blaustein AR (1998). Sex recognition and mate choice by male western toads, Bufo boreas. Animal Behaviour 55(6): 1631-1635.

Mascheretti S, Rogatcheva MB, Gunduz I, Fredga K, Searle JB (2003). How did pygmy shrews colonize Ireland? Clues from a phylogenetic analysis of mitochondrial chytochrome b sequences. Proceedings of the Royal Society of London, B Biological Science 270: 1593-1599.

Maynard Smith J, Haigh J (1974). The hitch-hiking effect of a favourable gene. Genetical Research 231: 1114-1116.

McCormick F (1999). Early evidence for wild animals in Ireland. In: Benecke N (ed) The Holocene history of the European vertebrate fauna. Modern aspects of research. Verlag Marie Leidorf GmbH: Germany, pp 355-371.

Merilä J, Crnokrak P (2001). Comparison of genetic differentiation at marker loci and quantitative traits. Journal of Evolutionary Biology 14: 892-903.

Mitchell F (1986). The Shell guide to reading the Irish landscape. Country House Press: Dublin.

Moritz C (1994). Defining 'Evolutionary Significant Units' for conservation. Trends in Ecology and Evoltution 9(10): 373-375.

Murphy FA, Fauquet CM, Bishop DHL, Ghabrial SA, Jarvis AW, Martelli GP et al (eds) (1995). Virus Taxonomy. Classification and Nomenclature of Viruses. Sixth Report of the International Committee on Taxonomy of Viruses. . Springer: New York.

Nei M (1987). Molecular Evolutionary Genetics Columbia University Press: New York. 
Nei M, Maruyama T, Chakraborty R (1975). The bottleneck effect and genetic variability in populations. Evolution 29: 1-10.

Nichols RA, Hewitt GM (1994). The genetic consequences of long distance dispersal during colonization. Heredity 72: 312-317.

Nonaka M, Namikawa C, Kato Y, Sasaki M, Salter-Cid L, Flajnik MF (1997). Major histocompatibility complex gene mapping in the amphibian Xenopus implies a primordial organization. Proceedings of the National Acadamy of Sciences USA 94: 5789-5791.

O'Rourke FJ (1970). The Fauna of Ireland. Mercier Press: Cork.

Ohta Y, Goetz W, Zulfiquer Hossain M, Nonaka M, Flajnik MF (2006). Ancestral Organization of the MHC Revealed in the Amphibian Xenopus. Journal of Immunology 176: $3674-3685$.

Ohtsuka M, Inoko H, Kulski JK, Yoshimura S (2008). Major histocompatibility complex (MHC) class Ib gene duplications, organization and expression patterns in mouse strain C57BL/6. BMC Genomics 9: 178.

Orlando L, Bonjean D, Bocherens H, Thenot A, Argant A, Otte M et al (2002). Ancient DNA and the population genetics of cave bears (Ursus spelaeus) through space and time. Molecular Biology and Evolution 19: 1920-1933.

Palo JU, Merilä J (2003). A simple RFLP method to identify two ranid frogs. Conservation Genetics 4: 801-803.

Palo JU, Schmeller DS, Laurila A, Primmer CR, Kuzmin SL, Merilä J (2004). High degree of population subdivision in a widespread amphibian. Molecular Ecology 13: 2631-2644.

Pamilo P (1984). Genotypic correlation and regression in social groups: multiple alleles, multiple loci and subdivided groups. Genetics 107: 307-320.

Pamilo P (1985). Effect of inbreeding on genetic relatedness. Hereditas 103: 195-200.

Pearman PB, Garner TWJ (2005). Susceptibility of Italian Agile Frog populations to an emerging Ranavirus parallels population genetic diversity. Ecology Letters 8: 401-408.

Pidancier N, Gauthier P, Miquel C, Pompanon F (2002). Polymorphic microsatellite DNA loci identified in the common frog (Rana temporaria, Amphibia, Ranidae). Molecular Ecology Notes 2: 304-305. 
Piertney SB, Oliver MK (2006). The evolutionary ecology of the major histocompatibility complex. Heredity 96: 7-21.

Platts EA, Speight MCD (1988). The taxonomy and distribution of the Kerry slug, Geomalacus maculosus Allman, 1843 (Mollusca: Arionidae) with a discussion of its status as a threatened species. Irish Naturalists' Journal 22: 417-430.

Posada D, Crandall KA (1998). Modeltest: testing the model of DNA substitution. Bioinformatics 14(9): 817-818.

Pounds JA, Bustamante MR, Coloma LA, Consuegra JA, Fogden MP, Foster PN et al (2006). Widespread amphibian extinctions from epidemic disease driven by global warming. Nature 439: 161-167.

Praeger RL (1939). The relations of the flora and fauna of Ireland to those of other countries. Proceedings of the Linnean Society 151: 192-213.

Preece RC, Coxon P, Robinson JE (1986). New biostratigraphic evidence of post-glacial colonisation of Ireland and for Mesolithic forest disturbance. Journal of Biogeography 13: 487-509.

Queller DC, Goodnight KF (1989). Estimating relatedness using molecular markers. Evolution 43: 258-275.

Queney C, Ferrand N, Marchandeau S, Azevedo M, Mougel F, Branco M et al (2000). Absence of a genetic bottleneck in a wild rabbit (Oryctolagus cuniculus) population exposed to a severe viral epizootic. Molecular Ecology 9: 1253-1264.

Rasbash J, Steele F, Browne W, Prosser B. (2004). Centre for Multilevel Modelling, University of Bristol.

Raymond M, Rousset F (1995). GENEPOP (version 1.2): population genetics software for exact tests and ecumenicism. Journal of Heredity 86: 248-249.

Reh W, Seitz A (1990). The influence of land-use on the genetic structure of populations of the common frog Rana temporaria. Biological Conservation 54: 239-249.

Retallick RWR, McCallum H, Speare R (2004). Endemic infection of the amphibian chytrid fungus in a frog community post-decline. PLoS Biology 2: e351.

Rice WR (1989). Analyzing tables of statistical tests. Evolution 43(223-225).

Robert J, Morales H, Buck W, Cohen N, Marr S, Gantress J (2005). Adaptive immunity and histopathology in frog virus 3-infected Xenopus. Virology 20: 667-675. 
Roca AL, Georgiadis N, Pecon-Slattery J, O'Brien SJ (2001). Genetic evidence for two species of elephant in Africa. Science 293: 1473-1477

Roelants K, Gower DJ, Wilkinson M, Loader SP, Biju SD, Guillaume K et al (2007). Global patterns of diversification in the history of modern amphibians. PNAS 104(3): 887-892.

Rowe G, Beebee JC (2001). Polymerase chain reaction primers for microsatellite loci in the common frog Rana temporaria. Molecular Ecology Notes 1: 6-7.

Rowe G, Harris DJ, Beebee JC (2006). Lusitania revisited: A phylogeographic analysis of the natterjack toad Bufo calamita across its entire biogeographical range. Molecular phylogenetics and evolution 39: 335-346.

Rowe L, Houle D (1996). The lek paradox and the capture of genetic variance by condition dependent traits. Proceedings of the Royal Society of London, B 263: 14151421.

Rozen S, Skaletsky H (2000). Primer3 on the WWW for general users and for biologist programmers. In: Krawetz S and Misener S (eds) Bioinformatics Methods and Protocols: Methods in Molecular Biology. Humana Press: Totowa, NJ, pp 365-386.

Sagvik J, Uller T, Olsson M (2005). Outbreeding depression in the common frog, Rana temporaria. Conservation Genetics 6: 205-211.

Sammut B, Du Pasquier L, Ducoroy P, Laurens V, Marcuz A, Tournefier A (1999). Axolotl MHC architecture and polymorphism. European Journal of Immunology 29(9): 2897-2907.

Savage RM (ed) (1961). The Ecology and Life History of the Common Frog (Rana temporaria temporaria). Pitman: London.

Seddon JM, Ellegren H (2004). A temporal analysis shows major histocompatibility complex loci in the Scandinavian wolf population are consistent with neutral evolution. Proceedings of the Royal Society of London, B 271: 2283-2291.

Shepherd LD, Lambert DM (2008). Ancient DNA and conservation: lessons from the endangered kiwi of New Zealand. Molecular Ecology 17: 2174-2184.

Shum BP, Avila D, Du Pasquier L, Kasahara M, Flajnik MF (1993). Isolation of a classical MHC class I cDNA from an amphibian : evidence for only one class I locus in the Xenopus MHC. Journal of Immunology 151: 5376-5386.

Skellam TG (1951). Random dispersal in theoretical populations. Biometrika 38: 196218. 
Slate J, Gemmell NJ (2004). Eve 'n' Steve: recombination of human mitochondrial DNA. Trends in Ecology and Evolution 19(11): 561-563.

Smith M (1964). The British Amphibians and Reptiles, 3rd edn, Vol 20. Collins: London.

Snell C, Tetteh J, Evans IH (2005). Phylogeography of the pool frog (Rana lessonae Camerano) in Europe: evidence for native status in Great Britain and for an unusual postglacial colonization route Biological Journal of the Linnean Society 85(1): 41-51.

Sokal RR, Rohlf FJ (1995). Biometry: the principles and practice of statistics in biological research. W. H. Freeman and Co: New York, 887pp.

Steward JR, Lister AM (2001). Cryptic northern refugia and the origin of modern biota. Trends in Ecology and Evoltution 16: 608-613.

Stuart AJ, Van Wijngaarden-Bakker L (1985). Quaternary vertebrates. In: Edwards KJ and Warren WP (eds) The Quaternary History of Ireland. Academic Press: London.

Stuart SN, Chanson JS, Cox NA, Young BE, Rodrigues ASL, Fischman DL et al (2004). Status and trends of amphibian declines and extinctions worldwide. Science 306: 17831786.

Taberlet P, Fumagalli L, Wust-Saucy A-G, Cosson J-F (1998). Comparative phylogeography and postglacial colonization routes in Europe. Molecular Ecology 7: 453-464.

Tamura K, Nei M (1993). Estimation of the number of nucleotide substitutions in the control region of mitochondrial DNA in humans and chimpanzees. Molecular Biology and Evolution 10: 512-526.

Tanaka T, Matsui M, Takenaka O (1994). Estimation of phylogenetic relationships among Japanese brown frogs from mitochondrial cytochrome b gene (Amphibia, Anura). Zoological Science (Tokyo) 11: 753-757.

Tanaka T, Matsui M, Takenaka O (1996). Phylogenetic relationships of Japanese Brown frogs (Rana, Ranidae) assessed by mitochondrial cytochrome b sequences. Biochemical Systematics and Ecology 24: 299-307.

Trachtenberg E, Korber B, Sollars C, Kepler TB, Hraber PT, Hayes E et al (2003). Advantage of rare HLA supertype in HIV disease progression. Nature Medicine 9: 928935. 
Van den Bosh F, Zadocks JC, Metz JA (1988). Focus expansion in plant disease. II: Realistic parameter-sparse models. Phytopathology 78: 59-64.

Vyas JM, Van der Veen AG, Ploegh HL (2008). The known unknowns of antigen processing and presentation. Nature Reviews Immunology 8(8): 607-618.

Wahlund S (1928). Zusammensetzung von Population und Korrelationserscheinung vom Standpunkt der Vererbungslehre aus betrachtet. Hereditas 11: 65-106.

Wang J (2004). Sibship reconstruction from genetic data with typing errors. Genetics 166: $1963-1979$.

Watts PC, O’Leary D, Cross MC, Coughlan J, Dillane E, Kay SM et al (2008). Contrasting levels of genetic differentiation among putative neutral microsatellite loci in Atlantic herring Clupea harengus populations and the implications for assessing stock structure. Hydrobiologia 606(1): 27-33.

Webb T, Bartlein PJ (1992). Global changes during the last 3 million years: climatic controls and biotic response. Annual Review of Ecology and Systematics 23: 141-173.

Weir BS, Cockerham CC (1984). Estimating F-statistics for the analysis of population structure. Evolution 38: 1358-1370.

Whitaker RJ (1998). Island Biogeography. Oxford University Press, 416pp.

Wingfield RTR (ed) (1995). A model of seas levels in the Irish and Celtic seas during the end Pleistocene to Holocene transition. Geological Society Special Publication No.96: London.

Woodman P, McCarthy M, Monaghan N (1997). The Irish quaternary fauna project. Quaternary Science Review 16: 129-159.

Wright S (1943). Isolation by distance. Genetics 28: 114-138.

Wright S (1950). The genetical structure of populations. Nature 166(4215): 247-249.

Yalden D (ed) (1999). The history of British mammals. Poyser: London.

Zeisset I, Beebee TJC (2001). Determination of biogeographical range: an application of molecular phylogeography to the European pool frog Rana lessonae. Proceedings of the Royal Society of London, B Biological Science 268: 933-938.

Zhang Q-Y, Xiao F, Li Z-Q, Gui J-F, Mao J, Chinchar VG (2001). Characterization of an iridovirus from the culture pig frog (Rana grylio) with lethal syndrome. Diseases of Aquatic Organisms 48(1): 27-36. 


\section{Appendix 1 -Protocol for cleaning sequencing reaction products}

1. Centrifuge reaction product @ 100g/60s

2. Add $3.75 \mu 1125 \mathrm{mM}$ EDTA per reaction

3. Add $45 \mu 1100 \%$ ethanol per reaction

4. Seal and invert plate 4 times

5. Incubate@ room temperature for 15 minutes

6. Centrifuge @ 3000xg for 30 minutes $\left(4^{\circ} \mathrm{C}\right)$

7. IMMEDIATELY invert on tissue paper and centrifuge @ 100g for 60s

8. Add $45 \mu 170 \%$ ethanol per reaction

9. Seal and centrifuge @ 1650xg for 15 minutes $\left(4^{\circ} \mathrm{C}\right)$

10. Invert on tissue paper and centrifuge @ 100xg for 60s

11. Incubate at $55^{\circ} \mathrm{C}$ for 2 minutes to evaporate any remaining ethanol

12. Keep pellet frozen prior to re-suspension in $10 \mu 1$ of Formamide 
Appendix 2 - Polymorphic sites at each mtDNA haplotype.

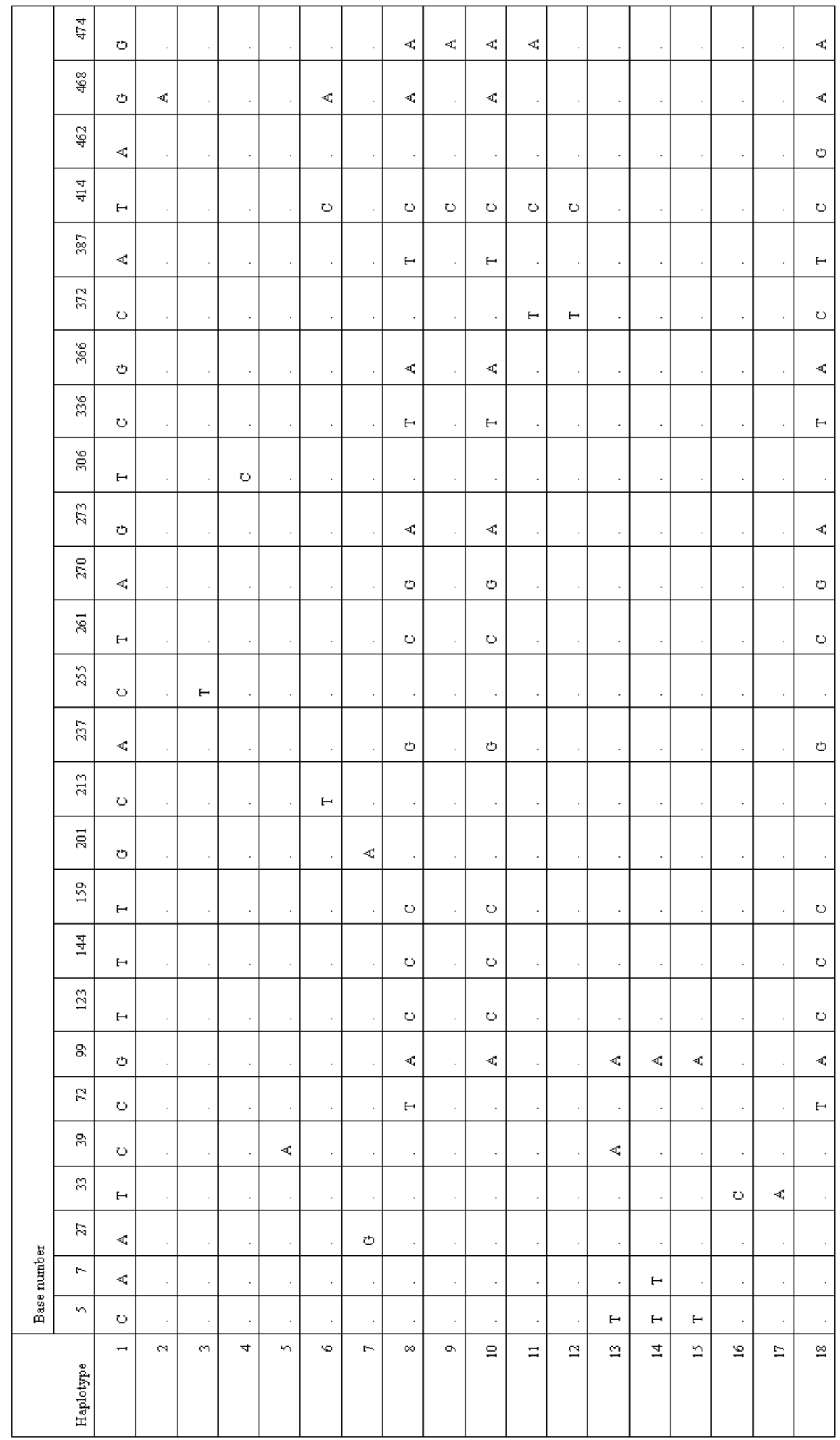




\section{Appendix 3 - Simulation code for use in $\mathbf{R}$ package}

\# Cleanup existing variables

$\operatorname{rm}(\operatorname{list}=\operatorname{ls}())$

\# Package for resampling

library(gdata)

\# example of a random vector of 1 and 2

\# resample(c(1,2),5, replace $=\mathrm{T})$

\# Basic parameters

NumPairs $<-30$

Survival<-1/3 \# Proportion of breeding adults surviving to next gen

NumLoci<-14

MaxAlleles $<-20$

Pres<-.3 \# Frequency of the resistance allele

Fassort<-.99 \# Strength of assortative mating

\# Arrays to hold genetic data

GrandParents<-

array(resample((1:MaxAlleles),NumPairs*NumLoci*4,replace=T),dim=c(NumPairs,2,NumLoci, 2))

Parents<array(resample((1:MaxAlleles),NumPairs*NumLoci*4,replace=T), dim=c(NumPairs,2,NumLoci, 2))

\# Fill the first locus in the array with resistance alleles at frequency Pres

GrandParents[,,1,]<-rbinom(NumPairs*4,1,Pres)

Parents $[,, 1]<$,- rbinom(NumPairs*4,1,Pres)

\# Function to create a new individual from the breeding generation ( 2 seasons ago)

offspring<-function(GrandParents,MaleChoices) \{

mum<-ceiling(runif(1, max=NumPairs)

dad<-MaleChoices[mum]

cbind( GrandParents[mum,1,,][cbind(1:NumLoci,ceiling(runif(NumLoci,max=2)))],

GrandParents[dad,2,,][cbind(1:NumLoci,ceiling(runif(NumLoci,max=2)))]) 
\}

\# Function to create new breeding generation

createNewParents<-function(GrandParents,Parents)\{

\# Work out which mums and dads are resistant (carry at least one resistance allele)

MumResistant<-rowSums(GrandParents[,1,1,])>0

DadResistant<-rowSums(GrandParents[,2,1,])>0

\# Choose mates for females with probability proportional to Fassort if they share resistance status \# and (1-Fassort) if they don't

MaleChoices<-vector(length=NumPairs)

\# Allocate mates for resistant females

MaleChoices[MumResistant]<sample(1:NumPairs,sum(MumResistant),replace $=\mathrm{T}, \mathrm{prob}=(($ DadResistant $*$ Fassort $)+((!$ DadResista $\mathrm{nt}) *(1-$ Fassort $))))$

\# Allocate mates for susceptible females

MaleChoices[!MumResistant]<sample(1:NumPairs,sum(!MumResistant),replace $=\mathrm{T}, \mathrm{prob}=(((!$ DadResistant $) *$ Fassort $)+($ DadResis $\tan *^{*}(1-$ Fassort $\left.\left.\left.)\right)\right)\right)$

\# Create the new population (NP)

$\mathrm{NP}<-\operatorname{array}(\operatorname{dim}=\mathrm{c}($ NumPairs,2,NumLoci,2))

for (P in 1:NumPairs) for (Sex in 1:2)\{

\# with probability survival the new parent is a surviving breeder from the previous generation

if (runif(1)<Survival)

NP[P,Sex,,]<-Parents[ceiling(runif(1,max=NumPairs)),Sex,,]

\# Otherwise it is a new recruit from breeding

else $\quad \mathrm{NP}[\mathrm{P}, \mathrm{Sex},]<$,

offspring(GrandParents,MaleChoices) \}

NP

\}

\# Function to create sample from clutches

createSample<-function(GrandParents) \{

\# Work out which mums and dads are resistant (carry at least one resistance allele)

MumResistant<-rowSums(GrandParents[,1,1,])>0

DadResistant<-rowSums(GrandParents[,2,1,])>0

\# Choose mates for females with probability proportional to Fassort if they share resistance status \# and (1-Fassort) if they don't 
MaleChoices<-vector(length=NumPairs)

\# Allocate mates for resistant females

MaleChoices[MumResistant]<sample(1:NumPairs,sum(MumResistant),replace $=\mathrm{T}, \mathrm{prob}=(($ DadResistant $*$ Fassort $)+((!$ DadResista $\mathrm{nt}) *(1-$ Fassort $))))$

\# Allocate mates for susceptible females

MaleChoices[!MumResistant]<sample(1:NumPairs,sum(!MumResistant),replace $=\mathrm{T}$, prob $=(((!$ DadResistant $) *$ Fassort $)+($ DadResis $\operatorname{tant} *(1-$ Fassort $))))$

$\mathrm{NP}<-\operatorname{array}(\operatorname{dim}=\mathrm{c}($ NumPairs,NumLoci,2) $)$

for (mum in 1:NumPairs) \{

\# find dad

dad<-MaleChoices[mum]

\# choose 1 egg from each clutch

$\mathrm{NP}[$ mum,,$<-$-cbind (

GrandParents[mum,1,,][cbind(1:NumLoci,ceiling(runif(NumLoci,max=2)))],

GrandParents[dad,2,,][cbind(1:NumLoci,ceiling(runif(NumLoci,max=2)))])

\}

NP

\}

\# Function to calculate Fis according to Weir \& Cockerham

Fis $<$-function(sample) \{

$\mathrm{P}<-\operatorname{array}(0, \operatorname{dim}=\mathrm{c}($ NumLoci,MaxAlleles $))$

$\mathrm{H}<-\operatorname{array}(0, \operatorname{dim}=\mathrm{c}($ NumLoci,MaxAlleles $))$

$\mathrm{N}<-$ NumPairs

\# Calculate allele frequencies for each locus

for (L in 1:NumLoci) for (A in 1:MaxAlleles) $\mathrm{P}[\mathrm{L}, \mathrm{A}]<-\operatorname{sum}($ sample$[, \mathrm{L}]=,=\mathrm{A}$ )

$\mathrm{P}<-\mathrm{P} / \mathrm{N} / 2$

\# Calculate heterozygosity for each allele at each locus

for (A in 1:MaxAlleles) H[,A]<-colSums $(($ sample[,,1]==A)+(sample[,,2]==A)==1))

$\mathrm{H}<-\mathrm{H} / \mathrm{N}$

$\mathrm{H}<-\mathrm{H}[2:$ NumLoci,

$\mathrm{P}<-\mathrm{P}[2:$ NumLoci, $]$ 
\# calculate $\mathrm{b} \& \mathrm{c}$ for in W\&C's formula for Fis

$\mathrm{c}<-\operatorname{sum}(\mathrm{H} / 2)$

$\mathrm{b}<-\operatorname{sum}((\mathrm{P} *(1-\mathrm{P})-(2 * \mathrm{~N}-1) * \mathrm{H} / 4 / \mathrm{N}) * \mathrm{~N} /(\mathrm{N}-1))$

$1-c /(b+c)$

\}

\# Function to calculate heterozygosity

heterozygosity<-function(S) \{sample<-createSample(GrandParents)

$\mathrm{P}<-\operatorname{array}(0, \operatorname{dim}=\mathrm{c}($ NumLoci,MaxAlleles $))$

$\mathrm{N}<-$ NumPairs

SubSample<-createSample(S)

\# Calculate allele frequencies for each locus

for (L in 1:NumLoci) for (A in 1:MaxAlleles) $\mathrm{P}[\mathrm{L}, \mathrm{A}]<-\operatorname{sum}($ SubSample[,L,]==A)

$\mathrm{P}<-\mathrm{P} / \mathrm{N} / 2$

$1-\operatorname{rowSums}\left(\mathrm{P}^{\wedge} 2\right)$

\}

res $<-$ vector(length=1000)

for (i in 1:1000) \{

GrandParents<-

array(resample((1:MaxAlleles),NumPairs*NumLoci*4,replace=T),dim=c(NumPairs,2,NumLoci, 2))

Parents<-

array(resample((1:MaxAlleles),NumPairs*NumLoci*4,replace=T),dim=c(NumPairs,2,NumLoci, 2))

GrandParents[,,1,]<-rbinom(NumPairs*4,1,Pres)

Parents[,,1,]<-rbinom(NumPairs*4,1,Pres)

for (year in 1:10)\{

$\mathrm{P}<$-Parents

Parents<-createNewParents(Parents, GrandParents)

GrandParents $<-\mathrm{P}\}$

res[i]<-Fis(createSample(GrandParents))

\}

hist(res)

mean(res)

$\operatorname{sd}($ res $)$ 


\section{Appendix 4 - $\mathbf{R}$ code for randomization of disease status and generation of Chi- squared distribution}

dat<-read.table(header=T,'MHCcomb.txt')

$\operatorname{attach}($ dat $)$

Pop<-as.factor(Pop)

Nalleles<-nlevels(Allele)

$\mathrm{D}<-$ matrix $($ nrow $=$ Nalleles, ncol $=2$ )

for ( $\mathrm{r}$ in 1:Nalleles) $\mathrm{D}[\mathrm{r}, 1]=\operatorname{sum}(($ Allele$==\operatorname{levels}($ Allele $)[\mathrm{r}]) \&($ Disease $==" \mathrm{p} "))$

for $(\mathrm{r}$ in 1:Nalleles) $\mathrm{D}[\mathrm{r}, 2]=\operatorname{sum}(($ Allele $==\operatorname{levels}($ Allele $)[\mathrm{r}]) \&$ !(Disease $==" \mathrm{p} "))$

chisq.test $(\mathrm{D}$, simulate.p.value $=\mathrm{T}, \mathrm{B}=10000)$

numiters $<-10000$

ChiVals<-vector(length=numiters)

for (iter in 1:numiters) \{

tempD<-is.element(Pop,sample(levels(Pop),7))

for ( $\mathrm{r}$ in 1:Nalleles) $\mathrm{D}[\mathrm{r}, 1]=\operatorname{sum}(($ Allele $==\operatorname{levels}($ Allele $)[\mathrm{r}]) \&$ tempD)

for ( $\mathrm{r}$ in 1:Nalleles) $\mathrm{D}[\mathrm{r}, 2]=\operatorname{sum}(($ Allele==levels (Allele $)[\mathrm{r}]) \&$ !tempD)

ChiVals[iter]<-chisq.test(D)\$statistic \}

quantile(ChiVals,c(0.5,0.95,0.99))

hist(ChiVals) 


\section{Appendix 5 - Parliamentary Office of Science and Technology note}

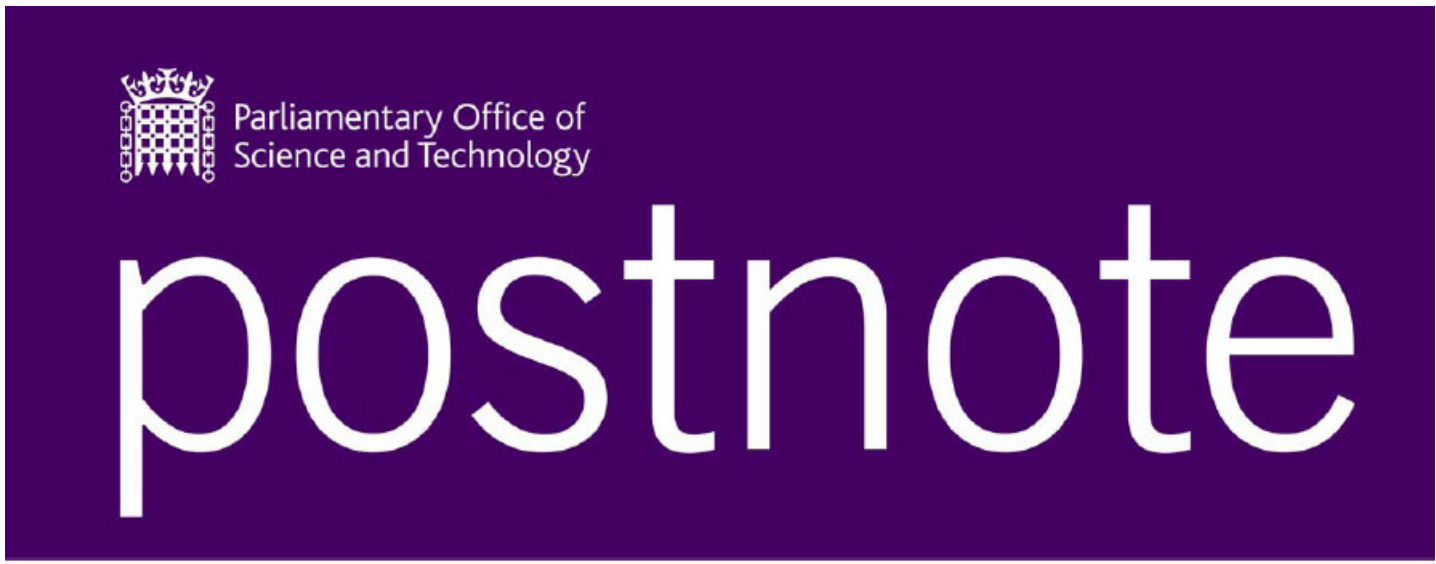

April 2008 Number 307

\section{WILDLIFE DISEASES}

Wildlife diseases can have negative consequences for biodiversity, human and livestock health, animal welfare and the economy. At present UK wildlife disease surveillance is poorly coordinated. The lack of a cohesive approach stems from a division of responsibility and a dominant focus on livestock and human health. This POSTnote examines the impacts of wild life diseases, the current status of surveillance in the UK and the options to strengthen policies.

\section{Background}

Many harmful biological agents (pathogens) can infect a broad range of species, including humans. ${ }^{1}$ Wildlife play a major role in disease transmission and so is important when addressing certain diseases in domestic animals or humans. Wildlife diseases are also important in their own right, with impacts on biodiversity and animal welfare. Climate change is expected to lead to substantial changes in wildlife disease patterns and frequency. ${ }^{2}$ As conservation programmes expand and contact between humans, domestic animals and wildlife increases, conflicts between biodiversity conservation, public health and domestic animal health may intensify. ${ }^{3}$

Historically wildlife diseases have usually been of interest after they have directly impacted livestock or human health. Because of this, responsibility for their surveillance and management is spread between many organisations. The Department for Environment, Food and Rural Affairs (Defra) is the government agency responsible for environmental issues. Defra employs one wildlife health veterinarian but has no specific budget dedicated to the subject

\section{Impacts of Wildlife Disease}

\section{Human healt}

Zoonotic diseases are diseases of animals that can be transmitted to humans; for example avian influenza, anthrax and rabies. Wildlife plays a key role by providing a 'zoonotic pool' from which new diseases may emerge.
The majority ( $60 \%$ ) of emerging infectious diseases in humans are caused by zoonotic pathogens and $72 \%$ of these have a wildlife origin. Human encroachment on shrinking wildlife habitats can cause increased wildlife population densities which can boost disease transmission risks. ${ }^{2,4}$ Also, increased human population density is linked to a rise in the number of zoonotic infections in humans ${ }^{4}$

Domestic animal health

Domestic animal disease can have serious economic effects. For instance, the 2001 Foot and Mouth Disease outbreak is estimated to have cost the UK $£ 1.2$ billion. Most $(77 \%)$ infectious diseases of domestic animals are common to wildlife ${ }^{5}$, so the control of a disease in domestic animals can be impeded by its presence in wildlife. Movement of domestic animals for trade and farming can help to spread disease. While culling infected livestock can reduce levels of disease, if the disease exists in wildlife it can be passed back to domestic animals at a later point.

\section{Biodiversity}

It is increasingly accepted that diseases can affect biodiversity and contribute to species declines (Box 1) ${ }^{6,7}$ For example, squirrel poxvirus is contributing to the decline of the red squirrel population, and crayfish plague is considered responsible for declines in native whiteclawed crayfish numbers (POSTnote 303) ${ }^{6}$ Diseases also impact on conservation efforts. For example in 1995, a release of captive-bred field crickets in England was suspended for two years due to infection with a parasite which posed a possible threat to other wild species. ${ }^{2}$

\section{Animal welfare}

Diseases such as mange in foxes and myxomatosis in rabbits (Box 1) can impact on the welfare of wild animals. In the UK, there is a great level of public concern regarding livestock, laboratory animal and pet welfare. Disease is a normal process in nature but human 
interventions can directly cause disease outbreaks in wildlife. In these cases, the Universities Federation for Animal Welfare states that we have a moral obligation to address the consequences.

\section{Box 1. Myxomatosis}

In the early 1950s, the myxomatosis virus was deliberately introduced to Europe as a bio-control agent for the population management of rabbits. Current estimates are that approximately 20 million rabbits are infected in Europe each year, causing tumours and high mortality. The introduction of myxomatosis has had a major welfare impact on wild European rabbits, and on pet and farmed rabbits.

The introduction of myxomatosis to Britain resulted in unintentional population declines in stoats, buzzards and owls. ${ }^{2}$ It also led to extinction in the UK of the large blue butterfly 30 years later through a series of complex ecological interactions:

\section{Disease surveillance}

Surveillance allows the identification of new infections and changes to existing ones. However, it is not economically viable to survey all species for all diseases. Certain species called 'sentinels' can be screened for diseases as indicators of the health status of a broader range of species. While sentinels may offer a cheaper option to surveillance of a wide range of species, their use is limited to certain known diseases in defined species. $^{8}$

EU surveillance obligations

There are no EU directives addressing disease monitoring for nature and biodiversity. There are disease specific directives and some generalist directives for agriculture and food safety. ${ }^{9}$ The World Organisation for Animal Health (OIE) is the intergovernmental organisation responsible for animal health surveillance worldwide. The OIE is under the control of an international committee consisting of delegates designated by the Governments of 172 member countries. The OIE lists diseases of perceived risk, termed 'notifiable' diseases. The UK is obliged to compile an annual report for the OIE on the presence of notifiable diseases and diseases with significant mortality or zoonotic potential. Annual OIE reports are analysed for the appearance of new diseases or changes in disease trends.

\section{Government surveillance in the UK}

Surveillance and management schemes are in place in the UK for specific notifiable diseases which impact on human or livestock health. Nationwide reporting mechanisms for wildlife mortalities and surveillance for non-notifiable diseases are limited.

The Veterinary Laboratories Agency (VLA), an executive agency of Defra, is primarily responsible for livestock diseases. It has a small budget and limited capacity for wildlife disease surveillance via the Diseases of Wildlife Scheme, which examines several hundred wild animal carcasses each year. Approximately $2 \%$ of the VLA's scanning (assessment of carcasses) surveillance budget is spent on wildlife health surveillance.
The Central Science Laboratory (CSL), also an executive agency of Defra, is primarily responsible for ensuring environmentally sustainable food production. The CSL provides surveillance of wildlife deaths caused by pesticides via the Wildlife Incident Investigation Scheme. The VLA issues questionnaires to institutions that work on wildlife to collate information for the OIE reports. However, not all laboratories and research groups submit records of wildlife diseases to the report, or have the time and resources to produce full reports of their activities and findings. ${ }^{10}$ As culling is the most widespread disease management strategy, the Royal Society for the Prevention of Cruelty to Animals states that some organisations may be reluctant to submit samples to government laboratories. This is likely to result in an underestimation of disease prevalence

\section{Non-governmental surveillance}

Surveillance and control of diseases at a local level is best addressed by veterinarians and wildlife workers with specialist expertise ${ }^{11}$ Individuals and NGOs such as the Zoological Society of London and the Wildlife Veterinary Investigation Centre perform a large proportion of UK wildlife disease surveillance and management. Population monitoring occurs as part of research and conservation projects, and within the environmental consultancy sector. Many organisations hold samples that could be used for retrospective surveillance (Box 2) However, a lack of integration between disciplines means that data are not shared

\section{Box 2. Retrospective surveillance}

Well maintained archives of tissue taken from dead wildlife would allow large numbers of samples to be screened quickly without expensive and laborious sample collection. The Central Science Laboratory (CSL) archives wildlife tissues after post-mortem examinations and maintains a database of these samples. The VLA and many nongovernment laboratories throughout the UK also have wildlife tissues in storage. The CSL has plans for a centralised web-based database of wildlife tissue samples, but no funds are available.

The role of the public in surveillance

The public has a great level of interest in wildlife; over 400,000 volunteers took part in the 2007 Royal Society for the Protection of Birds 'Big Garden Birdwatch'. Widespread public education may be a valuable method of reducing disease risks. Defra is keen to encourage the public to report incidents but limited resources mean that not all samples submitted are accepted by government laboratories. The Institute of Zoology believes that the public are undervalued as a surveillance tool Responsible media coverage is important as there is a risk of public antagonism towards wildlife due to a perceived threat to human health. ${ }^{12}$

\section{Predicting trends in wildlife disease} Assessing risks posed by future wildlife disease threats has been identified by the CSL as a weakness in the UK. Zoonotic disease risks are assessed by the joint Human Animal Infections and Risk Surveillance group, with 
members from the Health Protection Agency, Defra, VLA, and the Department of Health. The VLA and CSL receive funds from Defra for specific disease or species issues but no core funds for horizon scanning. A key issue is climate change, which is expected to cause diseases to spread to new areas as many pathogens survive better at warmer temperatures.

\section{Disease management \\ How diseases are spread}

Movements of animals provide a route for the transfer of pathogens between animals and the spread of diseases to new areas. ${ }^{2,3,11}$ Movement of animals occurs for the pet, laboratory, food, farming and hunting trades as well as for conservation, reintroductions and rehabilitation (Box 3). These trades are large, increasingly globalised and without adequate disease surveillance ${ }^{6}$ Globally, outbreaks resulting from wildlife trade have caused hundreds of billions of dollars of economic damage. ${ }^{5}$ Increasing human and pet travel are also partly responsible for the spread of diseases. ${ }^{3}$

\section{Box 3. Wildlife rehabilitation}

Wildlife rehabilitation involves the collection of diseased or injured wildlife, euthanasia, or treatment and release into the wild. Defra estimates that there are about 700 animal sanctuaries in the UK. Of particular concern to the British Wildlife Rehabilitation Council (BWRC) is the potential for rehabilitated badgers to spread bovine tuberculosis (bTB). No bTB screening is required by law, and badgers can be released in an area different from that where they were found. There is as yet no reliable test available for bTB in badgers so the only effective risk mitigation strategy is to release solely at the place of capture. The BWRC issues guidelines on preventing infections in units but there is currently no obligation to perform disease screening, or to contribute to disease surveillance. A licensing system for the release of non-native species and species listed on Schedule 9 of the Wild life and Countryside Act (1981) exists but there is currently no legislation regulating the movement of native species or re-introductions.

\section{Limiting transmission of disease}

Preventing diseases entering and spreading in animal populations is the most efficient and cost effective way of managing disease. ${ }^{13}$ Natural England issues licences for movement of some protected species, for example the great crested newt. Defra reviews licence applications from a veterinary perspective to assess known disease risks. While many approaches to management are disease specific, improved regulation of movements of animals by people may provide broader protection. However, regulation of animal movements between countries could impede international trade. Regulation of animal movements within the UK is logistically difficult to enforce but could act as a deterrent if not a solution.

\section{Controlling outbreaks}

Wildlife deaths are often not apparent until a mass mortality has occurred, by which time it may be too late for successful management. Modelling disease outbreaks and spread can provide valuable information for the development of management strategies (Box 4).

\section{Box 4. Modelling diseases}

Modelling involves studying disease distribution and patterns of spread to determine the scale of a problem. This information is used to develop a model which can predict the spread of disease. Modelling can be used to assess potential disease impacts and develop contingency plans. For example, modelling was used to show that red squirrel population declines are 17-25 times higher in regions where squirrel poxvirus (carried by grey squirrels) is present. ${ }^{14}$ The model predicted that a grey squirrel population control where more than $60 \%$ are killed would stop red squirrel declines in Cumbria. Disease modelling requires prior knowledge of animal population distributions and ecology, diseases present and methods of disease transmission. Th strength of modelling is in the comparison of management options; it can help to identify where resources would be best invested, potentially improving the cost-effectiveness of management schemes.

Existing methods of surveillance and management of human and livestock diseases are often not suitable for use in wildlife. ${ }^{2}$ Wildlife populations are usually too large for individual animal treatments. Vaccination of freeranging wildlife is difficult to implement and few suitable vaccines are available. In spite of this, vaccination of foxes has been successful in the control of rabies in Europe and North America. Vaccination and individual treatments are considered most valuable for controlling disease in endangered species

EU strategy dictates that if a livestock disease outbreak is detected, movements of livestock are restricted and an eradication programme is initiated. ${ }^{9}$ Disease outbreaks in aquatic species are addressed in a similar way (Box 5).

Box 5. Managing diseases in aquatic species Fish farming and angling rely on healthy wildlife as wild fish form a component of their stock and so are of direct. economic importance. The Centre for Environment, Fisheries \& Aquaculture Science (Cefas), an executive agency of Defra, has the legislative powers, financial resources and diagnostic power to deal with notifiable disease outbreaks. Diseases that show no symptoms in fish but that affect other wildlife may be an additional risk. Cefas considers that, despite contingency planning, a disease outbreak on the scale of the 2001 Foot and Mouth Disease epidemic may overwhelm the available resources. In August 2008 a new EU Directive will give Cefas more power to control new emerging diseases.

Culling is the most common management method for wildlife yet is often impractical, expensive and raises ethical issues. It can have unexpected side effects, for example a large-scale study found that removal of badgers in one area was linked with increased incidences of tuberculosis in cattle in adjoining areas. The removal of some badgers is thought to result in the remaining badgers ranging more widely and spreading disease further ${ }^{15}$ Husbandry education for livestock owners could help to reduce the contact rates between livestock and wildlife. However, while complete prevention of such contact may be desirable, it is usually not feasible. ${ }^{11}$ 


\section{Strengthening policy approaches}

Successful wildlife disease management policies rely on sound scientific evidence and require input from a range of specialists including researchers, veterinarians and government. At present, disease policy is reactive and opportunistic as opposed to pro-active and structured When novel diseases emerge in the UK, responses are hampered by disjointed policies and poorly defined responsibilities (Box 6). Much of the necessary expertise and infrastructure to address diseases in wildlife exists but remains uncoordinated and under funded.

Box 6. Amphibian disease in the UK

Chytridiomycosis, a fungal disease, is a significant threat to amphibians. In some regions it is estimated that $50 \%$ of amphibian species and $80 \%$ of individuals disappear within 6 months of disease introduction. ${ }^{10}$ It is likely that human movement of wildlife has resulted in the pandemic of chytridiomycosis. The fungus has been reported in amphibians in the pet trade, food industry, zoo animals, laboratory animals, and those used as bio-control agents. Treatment of infected amphibians and disinfection of captive environments is possible, but control within a natural environment is unlikely to be successful without severe disruption to the ecosystem. ${ }^{17}$

Chytridiomycosis was first recorded in the UK in 2004; the potential impacts are unknown. In 2006 proposals were sent to the OIE to list this disease as notifiable. This is expected to be approved in May 2008 and, if so, the disease would become notifiable in January 2009. The UK

chytridiomycosis surveillance scheme began in early 2008 funded by Natural England and the Zoological Society of London. The surveillance scheme aims to see whether the disease remains isolated in a few populations or whether it is now widespread. If it has spread widely, eradication is unlikely to be viable. Under World Trade Organisation agreements, Great Britain is unable to restrict trade in amphibians based on presence of chytridiomycosis without enacting comprehensive eradication programmes. Four yea passed from the initial reports of chytridiomycosis in the UK to the implementation of a surveillance system, in which time the disease is likely to have spread. Disease management decisions rely on the surveillance results.

In an attempt to coordinate wildlife disease policy better, Defra will publish a Wildlife Health Strategy in mid2008. This is expected to recommend that government intervention is warranted for the following purposes: protection of human or domestic animal health protection of biodiversity or conservation; to safeguard trade and the economy. The strategy's aims represent wider stakeholder recommendations:

- that the implications of the disease status of wildlife are considered more widely and a responsible approach to human/wildlife interactions is adopted;

- a proportionate, risk-based approach to wildlife disease surveillance and prevention is adopted, and where necessary, appropriate interventions are made; and,

- a holistic and coordinated approach to wildlife health across Government and interested parties is realised. There is a broad consensus from stakeholders, academics and government agencies on the need for Defra to coordinate an effective and unified national strategy. However, Defra is experiencing major budgetary and staffing cuts; there is a risk that the aims of the strategy will not be achieved if funding is not allocated to it. National and international structures for the rapid dissemination of reliable information between scientists, policy makers and the public are a key area for government intervention. ${ }^{18}$ Identification of where responsibilities lie is also important.

\section{Overview}

- There is a lack of awareness of the implications of wildlife diseases. They affect biodiversity, animal welfare, human health and livestock health.

- Disease management is often considered only once a disease has already become a problem. Alternatives include the development of predictive approaches and the regulation of animal movements.

- Better coordinated collaborations are required to provide an improved nationwide reporting mechanism for wildlife mortalities and an improved surveillance scheme for non-notifiable diseases.

- There is a consensus on the need for a unified strategy and increased funding

Endnotes

1 Cleaveland, S. et al. (2001) Philosophical Transactions of the Royal Society B: Biological Sciences 356, 991-999

2 Daszak, P. et al. (2000) Science $287,443-449$

3 Daszak, P. and Cunningham, A.A. (2003) Journal of Parasitology $89, \$ 37-\$ 41$

4 Jones, K. E et al, (2008) Nature 451(21), 990-994

5 Karesh, W. B. et al. (2005) Emerging Infectious Disease 11(7), 1001-1002

6 Cunningham, A.A. et al. (2003) Journal of Parasitology 89, S78583

7 Sainsbury, A.W. et al. (2001) Veterinary Record 148, 558-563

8 Halliday, J.E. B. et al. (2007) Journal of the Royal Society Interface 4, 973-984

9 For example EU directives 92/1 19/EEC, 2003/99/EC, 82/894/EEC

10 Professor Bennett, Dept. of Veterinary Pathology, University of Liverpool, in pers, comm.

11 Bengis R.G. et al. (2002) Scientific and Technical Review of the Office International des Epizooties 21 (1), 53-65

12 Sutherland, W.J. et al. (2008) Journal of Applied Ecology (AOP)

13 Wobeser, G. (2002) Scientific and Technical Review of the Office International des Eoizooties 21(1), 159-178

14 Rushton, S.P. et al. (2006) Epidemiology and Infection 134, 521 533

15 Environment, Food and Rural Affairs Committee, Fourth Report of Session 2007-08, Badgers and cattle TB: the final report of the Independent Scientific Group on Cattle TB.

16 Lips K.R. et al. (2006) Proceedings of the National Academy of Sciences 103, 3165-3170

17 Dr Cunningham, Institute of Zoology, in pers. comm.

18 Daszak, P. et al. (1999) Emerging Infectious Diseases 5(6), 735748

POST is an office of both Houses of Parliament, charged with providing ndependent and balanced analysis of public policy issues that have a basis in science and technology.

POST is grateful to Amber Teacher for researching this briefing, to the Natural Environment Research Council for funding her parliamentary fellowship, and to al contributors and reviewers. For further information on this subject, please contact the co-author, Dr Wentworth, at POST.

Parliamentary Copyright 2008

The Parliamentary Office of Science and Technology, 7 Millbank, London, SW1P 3JA; Tel: 0207219 2840; email: post@parliament.uk www.parliament.uk/parliamentary_offices/post/pubs2008.cfm 